\title{
Strichartz estimates for Schrödinger equations with variable coefficients and unbounded potentials
}

\author{
Haruya Mizutani*
}

\begin{abstract}
The present paper is concerned with Schrödinger equations with variable coefficients and unbounded electromagnetic potentials, where the kinetic energy part is a long-range perturbation of the flat Laplacian and the electric (resp. magnetic) potential can grow subquadratically (resp. sublinearly) at spatial infinity. We prove sharp (local-in-time) Strichartz estimates, outside a large compact ball centered at origin, for any admissible pair including the endpoint. Under the nontrapping condition on the Hamilton flow generated by the kinetic energy, global-in-space estimates are also studied. Finally, under the nontrapping condition, we prove Strichartz estimates with an arbitrarily small derivative loss without asymptotic flatness on the coefficients.
\end{abstract}

\section{Introduction}

In this paper, we study sharp (local-in-time) Strichartz estimates for Schrödinger equations with variable coefficients and unbounded electromagnetic potentials. More precisely, we consider the following Schrödinger operator:

$$
H=\frac{1}{2} \sum_{j, k=1}^{d}\left(-i \partial_{j}-A_{j}(x)\right) g^{j k}(x)\left(-i \partial_{k}-A_{k}(x)\right)+V(x), \quad x \in \mathbb{R}^{d},
$$

where $d \geq 1$ is the spatial dimension. Throughout the paper we assume that $g^{j k}, V$ and $A_{j}$ are smooth real-valued functions on $\mathbb{R}^{d}$ and that $\left(g^{j k}(x)\right)_{j, k}$ is symmetric and positive definite:

$$
\sum_{j, k=1}^{d} g^{j k}(x) \xi_{j} \xi_{k} \geq c|\xi|^{2}, \quad x, \xi \in \mathbb{R}^{d}
$$

with some $c>0$. Moreover, we suppose the following condition:

2010 Mathematics Subject Classification. Primary 35Q41,35B45; Secondary 35S30, 81Q20.

Key words and phrases. Schrödinger equation, Strichartz estimates, asymptotically flat metric, unbounded electromagnetic potentials.

${ }^{*}$ Research Institute for Mathematical Sciences, Kyoto University, Kyoto 606-8502, Japan. Current address: Department of Mathematics, Gakushuin University, 1-5-1 Mejiro, Toshima-ku, Tokyo 171-8588, Japan. E-mail: haruya@math.gakushuin.ac.jp. The author was partially supported by GCOE 'Fostering top leaders in mathematics', Kyoto University. 
Assumption 1.1. There exists $\mu \geq 0$ such that for any $\alpha \in \mathbb{Z}_{+}^{d}$,

$$
\begin{aligned}
\left|\partial_{x}^{\alpha}\left(g^{j k}(x)-\delta_{j k}\right)\right| & \leq C_{\alpha}\langle x\rangle^{-\mu-|\alpha|}, \\
\left|\partial_{x}^{\alpha} A_{j}(x)\right| & \leq C_{\alpha}\langle x\rangle^{1-\mu-|\alpha|}, \\
\left|\partial_{x}^{\alpha} V(x)\right| & \leq C_{\alpha}\langle x\rangle^{2-\mu-|\alpha|}, \quad x \in \mathbb{R}^{d} .
\end{aligned}
$$

Then, it is well known that $H$ admits a unique self-adjoint realization on $L^{2}\left(\mathbb{R}^{d}\right)$, which we denote by the same symbol $H$. By the Stone theorem, $H$ generates a unique unitary propagator $e^{-i t H}$ on $L^{2}\left(\mathbb{R}^{d}\right)$ such that the solution to the Schrödinger equation:

$$
i \partial_{t} u(t)=H u(t), t \in \mathbb{R} ;\left.\quad u\right|_{t=0}=\varphi \in L^{2}\left(\mathbb{R}^{d}\right),
$$

is given by $u(t)=e^{-i t H} \varphi$.

In order to explain the purpose of the paper, we recall some known results. Let us first recall well known properties of the free propagator $e^{-i t H_{0}}$, where $H_{0}=-\Delta / 2$. The distribution kernel of $e^{-i t H_{0}}$ is given explicitly by $(2 \pi i t)^{-d / 2} e^{i|x-y|^{2} /(2 t)}$ and $e^{-i t H_{0}} \varphi$ thus satisfies the dispersive estimate:

$$
\left\|e^{-i t H_{0}} \varphi\right\|_{L^{\infty}\left(\mathbb{R}^{d}\right)} \leq C|t|^{-d / 2}\|\varphi\|_{L^{1}\left(\mathbb{R}^{d}\right)}, \quad t \neq 0 .
$$

Moreover, $e^{-i t H_{0}}$ enjoys the following (global-in-time) Strichartz estimates:

$$
\left\|e^{-i t H_{0}} \varphi\right\|_{L^{p}\left(\mathbb{R} ; L^{q}\left(\mathbb{R}^{d}\right)\right)} \leq C\|\varphi\|_{L^{2}\left(\mathbb{R}^{d}\right)},
$$

where $(p, q)$ satisfies the following admissible condition:

$$
p \geq 2, \quad \frac{2}{p}=d\left(\frac{1}{2}-\frac{1}{q}\right), \quad(d, p, q) \neq(2,2, \infty) .
$$

Strichartz estimates imply that, for any $\varphi \in L^{2}, e^{-i t H_{0}} \varphi \in \bigcap_{q \in Q_{d}} L^{q}$ for a.e. $t \in \mathbb{R}$, where $Q_{1}=[2, \infty], Q_{2}=[2, \infty)$ and $Q_{d}=[2,2 d /(d-2)]$ for $d \geq 3$. These estimates hence can be regarded as $L^{p}$-type smoothing properties of Schrödinger equations, and have been widely used in the study of nonlinear Schrödinger equations (see, e.g., [8]). Strichartz estimates for $e^{-i t H_{0}}$ were first proved by Strichartz [31] for a restricted pair of $(p, q)$ with $p=q=2(d+2) / d$, and have been generalized for $(p, q)$ satisfying (1.1) and $p \neq 2$ by [14]. The endpoint estimate $(p, q)=(2,2 d /(d-2))$ for $d \geq 3$ was obtained by [19].

For Schrödinger operators with electromagnetic potentials, i.e., $H=\frac{1}{2}\left(-i \partial_{x}-A\right)^{2}+V$, (short-time) dispersive and (local-in-time) Strichartz estimates have been extended with potentials decaying at infinity [33, or growing at infinity [13, 34]. In particular, it was shown by [13, 34] that if $g^{j k}=\delta_{j k}, V$ and $A$ satisfy Assumption 1.1] with $\mu \geq 0$ and all derivatives of the magnetic field $B=d A$ are of short-range type, then $e^{-i t H} \varphi$ satisfies (short-time) dispersive estimates:

$$
\left\|\left.e^{-i t H} \varphi\right|_{L^{\infty}\left(\mathbb{R}^{d}\right)} \leq C|t|^{-d / 2}\right\| \varphi \|_{L^{1}\left(\mathbb{R}^{d}\right)},
$$

for sufficiently small $t \neq 0$. Local-in-time Strichartz estimates, which have the forms

$$
\left\|e^{-i t H} \varphi\right\|_{L^{p}\left([-T, T] ; L^{q}\left(\mathbb{R}^{d}\right)\right)} \leq C_{T}\|\varphi\|_{L^{2}\left(\mathbb{R}^{d}\right)}, \quad T>0,
$$

are immediate consequences of this estimate and the $T T^{*}$-argument due to Ginibre-Velo [14] (see Keel-Tao [19] for the endpoint estimate). For the case with singular electric potentials or with 
supercritical electromagnetic potentials, we refer to [33, 35, 37, 9] and reference therein. We mention that global-in-time dispersive and Strichartz estimates for scattering states have been also studied under suitable decaying conditions on potentials and assumptions for zero energy; see [18, 36, 29, 12, 10] and reference therein. We also mention that there is no result on sharp global-in-time dispersive estimates for magnetic Schrödinger equations.

On the other hand, the influence of the geometry on the behavior of solutions to linear and nonlinear partial differential equations has been extensively studied. From this geometric viewpoint, sharp local-in-time Strichartz estimates for Schrödinger equations with variable coefficients (or, more generally, on manifolds) have recently been investigated by many authors under several conditions on the geometry; see, e.g., [30, 6, 25, 15, 4, 3, 7, 23] and reference therein. In [30], 25], [4], the authors studied the case on the Euclidean space with nontrapping asymptotically flat metrics. The case on the nontrapping asymptotically conic manifold was studied by [15] and [23. In [3] the author considered the case of nontrapping asymptotically hyperbolic manifold. For the trapping case, it was shown in [6] that Strichartz estimates with a loss of derivative $1 / p$ hold on any compact manifolds without boundaries. They also proved that the loss $1 / p$ is optimal in the case of $M=\mathbb{S}^{d}$. In [4], 3] and [23], the authors proved sharp Strichartz estimates, outside a large compact set, without the nontrapping condition. More recently, it was shown in [7] that sharp Strichartz estimates still hold for the case with hyperbolic trapped trajectories of sufficiently small fractal dimension. We mention that there are also several works on global-in-time Strichartz estimates in the case of long-range perturbations of the flat Laplacian on $\mathbb{R}^{d}([5,32,22])$.

While (local-in-time) Strichartz estimates are well studied subjects for both of these two cases (at least under the nontrapping condition), the literature is more sparse for the mixed case. In this paper we give a unified approach to a combination of these two kinds of results. More precisely, under Assumption 1.1 with $\mu>0$, we prove (1) sharp local-in-time Strichartz estimates, outside a large compact set centered at origin, without the nontrapping condition; (2) the global-in-space estimates with the nontrapping condition. Under the nontrapping condition and Assumption 1.1 with $\mu \geq 0$, we also show local-in-time Strichartz estimates with an arbitrarily small derivative loss. We mention that all results include the endpoint estimates $(p, q)=(2,2 d /(d-2))$ for $d \geq 3$. This is a natural continuation of author's previous work [24, which was concerned with the non-endpoint estimates for the case with at most linearly growing potentials.

In the sequel, $F(*)$ denotes the characteristic function designated by $(*)$. We now state the main result.

Theorem 1.2 (Strichartz estimates near infinity). Suppose that $H$ satisfies Assumption 1.1 with $\mu>0$. Then, there exists $R_{0}>0$ such that for any $T>0, p \geq 2, q<\infty, 2 / p=d(1 / 2-1 / q)$ and $R \geq R_{0}$, we have

$$
\left\|F(|x|>R) e^{-i t H} \varphi\right\|_{L^{p}\left([-T, T] ; L^{q}\left(\mathbb{R}^{d}\right)\right)} \leq C_{T}\|\varphi\|_{L^{2}\left(\mathbb{R}^{d}\right)},
$$

where $C_{T}>0$ may be taken uniformly with respect to $R$.

To state the result on global-in-space estimates, we recall the nontrapping condition. Let us 
denote by $k(x, \xi)$ the classical kinetic energy:

$$
k(x, \xi)=\frac{1}{2} \sum_{j, k=1}^{d} g^{j k}(x) \xi_{j} \xi_{k},
$$

and by $\left(y_{0}(t, x, \xi), \eta_{0}(t, x, \xi)\right)$ the Hamilton flow generated by $k(x, \xi)$ :

$$
\dot{y}_{0}(t)=\partial_{\xi} k\left(y_{0}(t), \eta_{0}(t)\right), \dot{\eta}_{0}(t)=-\partial_{x} k\left(y_{0}(t), \eta_{0}(t)\right) ; \quad\left(y_{0}(0), \eta_{0}(0)\right)=(x, \xi) .
$$

Note that the Hamiltonian vector field $H_{k}=\partial_{\xi} k \cdot \partial_{x}-\partial_{x} k \cdot \partial_{\xi}$ generated by $k$ is complete on $\mathbb{R}^{2 d}$ since $\left(g^{j k}\right)$ satisfies the uniform elliptic condition. Hence, $\left(y_{0}(t, x, \xi), \eta_{0}(t, x, \xi)\right)$ exists for all $t \in \mathbb{R}$.

Definition 1.3. We say that $k(x, \xi)$ satisfies the nontrapping condition if for any $(x, \xi) \in \mathbb{R}^{2 d}$ with $\xi \neq 0$,

$$
\left|y_{0}(t, x, \xi)\right| \rightarrow+\infty \text { as } t \rightarrow \pm \infty .
$$

To control the asymptotic behavior of the flow, we also impose the following condition which is the classical analogue of Mourre's inequality:

Assumption 1.4 (Convexity near infinity). There exists $f \in C^{\infty}\left(\mathbb{R}^{d}\right)$ satisfying $f \geq 1$ and $f \rightarrow+\infty$ as $|x| \rightarrow+\infty$ such that $\partial^{\alpha} f \in L^{\infty}\left(\mathbb{R}^{d}\right)$ for any $|\alpha| \geq 2$ and

$$
H_{k}\left(H_{k} f\right)(x, \xi) \geq c k(x, \xi)
$$

on $\left\{(x, \xi) \in \mathbb{R}^{2 d} ; f(x) \geq R\right\}$ for some positive constants $c, R>0$.

Note that if $\left|\partial_{x} g^{j k}(x)\right|=o\left(|x|^{-1}\right)$ as $|x| \rightarrow+\infty$, then Assumption 1.4 holds with $f(x)=$ $1+|x|^{2}$. In particular, Assumption 1.1 with $\mu>0$ implies Assumption 1.4. Moreover, if $g^{j k}(x)=\left(1+a_{1} \sin \left(a_{2} \log r\right)\right) \delta_{j k}$ for $a_{1} \in \mathbb{R}, a_{2}>0$ with $a_{1}^{2}\left(1+a_{2}^{2}\right)<1$ and for $r=|x| \gg 1$, then Assumption 1.4 holds with $f(r)=\left(\int_{0}^{r}\left(1+a_{1} \sin \left(a_{2} \log t\right)\right)^{-1} d t\right)^{2}$. For more examples, we refer to [11, Section 2].

The second result then is the following.

Theorem 1.5 (Global-in-space Strichartz estimates). Suppose that $H$ satisfies Assumption 1.1 with $\mu \geq 0$. Let $T>0, p \geq 2, q<\infty$ and $2 / p=d(1 / 2-1 / q)$. Then, for any $r>0$, there exists $C_{T, r}>0$ such that

$$
\left\|F(|x|<r) e^{-i t H} \varphi\right\|_{L^{p}\left([-T, T] ; L^{q}\left(\mathbb{R}^{d}\right)\right)} \leq C_{T, r}\left\|\langle H\rangle^{\frac{1}{2 p}} \varphi\right\|_{L^{2}\left(\mathbb{R}^{d}\right)} .
$$

Moreover if we assume in addition that $k(x, \xi)$ satisfies the nontrapping condition (1.3) and that Assumption 1.4, then

$$
\left\|F(|x|<r) e^{-i t H} \varphi\right\|_{L^{p}\left([-T, T] ; L^{q}\left(\mathbb{R}^{d}\right)\right)} \leq C_{T, r}\|\varphi\|_{L^{2}\left(\mathbb{R}^{d}\right)} .
$$

In particular, combining with Theorem 1.2, we have (global-in-space) Strichartz estimates

$$
\left\|e^{-i t H} \varphi\right\|_{L^{p}\left([-T, T] ; L^{q}\left(\mathbb{R}^{d}\right)\right)} \leq C_{T}\|\varphi\|_{L^{2}\left(\mathbb{R}^{d}\right)},
$$

under the nontrapping condition (1.3), provided that $\mu>0$. 
For the general case we have the following partial result.

Theorem 1.6 (Near sharp estimates without asymptotic flatness). Suppose that $H$ satisfies Assumption 1.1 with $\mu \geq 0$ and $k(x, \xi)$ satisfies the nontrapping condition (1.3). Assume also Assumption 1.4. Let $T>0, p \geq 2, q<\infty$ and $2 / p=d(1 / 2-1 / q)$. Then, for any $\varepsilon>0$, there exists $C_{T, \varepsilon}>0$ such that

$$
\left\|e^{-i t H} \varphi\right\|_{L^{p}\left([-T, T] ; L^{q}\left(\mathbb{R}^{d}\right)\right)} \leq C_{T, \varepsilon}\left\|\langle H\rangle^{\varepsilon} \varphi\right\|_{L^{2}\left(\mathbb{R}^{d}\right)} .
$$

There are some remarks.

Remark 1.7. (1) The estimates of forms (1.2), (1.4) and (1.5) have been proved by [30, 4 ] when $A \equiv 0$ and $V$ is of long-range type. Theorems 1.2 and 1.5 hence are regarded as generalizations of their results for the case with growing electromagnetic potential perturbations.

(2) The only restriction for admissible pairs, in comparison to the flat case, is to exclude $(p, q)=$ $(4, \infty)$ for $d=1$, which is due to the use of the Littlewood-Paley decomposition.

(3) The missing derivative loss $\langle H\rangle^{\varepsilon}$ in Theorem 1.6 is due to the use of the following local smoothing effect (due to Doi [1]]):

$$
\left\|\langle x\rangle^{-1 / 2-\varepsilon}\langle D\rangle^{1 / 2} e^{-i t H} \varphi\right\|_{L^{2}\left([-T, T] ; L^{2}\left(\mathbb{R}^{d}\right)\right)} \leq C_{T, \varepsilon}\|\varphi\|_{L^{2}\left(\mathbb{R}^{d}\right)} .
$$

It is well known that this estimate does not holds when $\varepsilon=0$ even for $H=H_{0}$. We would expect that Theorem 1.2 still holds true for the case with critical electromagnetic potentials in the following sense:

$$
\langle x\rangle^{-1}\left|\partial_{x}^{\alpha} A_{j}(x)\right|+\langle x\rangle^{-2}\left|\partial_{x}^{\alpha} V(x)\right| \leq C_{\alpha \beta}\langle x\rangle^{-|\alpha|},
$$

(at least if $g^{j k}$ satisfies the bounds in Assumption 1.1 with $\mu>0$ ). However, this is beyond our techniques (see also remark 4.2).

The rest of the paper is devoted to the proofs of Theorems [1.2, 1.5 and 1.6. Throughout the paper we use the following notations: $\langle x\rangle$ stands for $\sqrt{1+|x|^{2}}$. We write $L^{q}=L^{q}\left(\mathbb{R}^{d}\right)$ if there is no confusion. For Banach spaces $X$ and $Y$, we denote by $\|\cdot\|_{X \rightarrow Y}$ the operator norm from $X$ to $Y$. We write $\mathbb{Z}_{+}=\mathbb{N} \cup\{0\}$ and denote the set of multi-indices by $\mathbb{Z}_{+}^{d}$. We denote by $K$ the kinetic energy part of $H$ and by $H_{0}$ the free Schrödinger operator:

$$
K=-\frac{1}{2} \sum_{j, k=1}^{d} \partial_{j} g^{j k}(x) \partial_{k}, \quad H_{0}=-\frac{1}{2} \Delta=-\frac{1}{2} \sum_{j=1}^{d} \partial_{j}^{2} .
$$

We set two symbols $p(x, \xi)$ and $p_{1}(x, \xi)$ defined by

$$
\begin{array}{r}
p(x, \xi)=\frac{1}{2} \sum_{j, k=1}^{d} g^{j k}(x)\left(\xi_{j}-A_{j}(x)\right)\left(\xi_{k}-A_{k}(x)\right)+V(x), \\
p_{1}(x, \xi)=-\frac{i}{2} \sum_{j, k=1}^{d}\left(\frac{\partial g^{j k}}{\partial x_{j}}(x)\left(\xi_{k}-A_{k}(x)\right)-g^{j k}(x) \frac{\partial A_{k}}{\partial x_{j}}(x)\right) .
\end{array}
$$

Note that Assumption 1.1 implies

$$
\begin{aligned}
\left|\partial_{x}^{\alpha} \partial_{\xi}^{\beta} p(x, \xi)\right| & \leq C_{\alpha \beta}\langle x\rangle^{-|\alpha|}\langle\xi\rangle^{-|\beta|}\left(|\xi|^{2}+\langle x\rangle^{2-\mu}\right), \\
\left|\partial_{x}^{\alpha} \partial_{\xi}^{\beta} p_{1}(x, \xi)\right| & \leq C_{\alpha \beta}\langle x\rangle^{-|\alpha|}\langle\xi\rangle^{-|\beta|}\left(\langle x\rangle^{-1-\mu}|\xi|+\langle x\rangle^{-\mu}\right) .
\end{aligned}
$$


For $h \in(0,1]$ we consider $H^{h}:=h^{2} H$ as a semiclassical Schrödinger operator with $h$-dependent electromagnetic potentials $h^{2} V$ and $h A_{j}$. The corresponding symbols $p_{h}$ and $p_{1, h}$ are also defined by

$$
\begin{gathered}
p_{h}(x, \xi)=\frac{1}{2} \sum_{j, k=1}^{d} g^{j k}(x)\left(\xi_{j}-h A_{j}(x)\right)\left(\xi_{k}-h A_{k}(x)\right)+h^{2} V(x), \\
p_{1, h}(x, \xi)=-\frac{i}{2} \sum_{j, k=1}^{d}\left(\frac{\partial g^{j k}}{\partial x_{j}}(x)\left(\xi_{k}-h A_{k}(x)\right)-h g^{j k}(x) \frac{\partial A_{k}}{\partial x_{j}}(x)\right) .
\end{gathered}
$$

It is easy to see that $H=\mathrm{Op}(p)+\mathrm{Op}\left(p_{1}\right)$ and $H^{h}=\mathrm{Op}_{h}\left(p_{h}\right)+h \mathrm{Op}_{h}\left(p_{1, h}\right)$.

Before starting the details of the proofs, we here describe the main ideas. At first we note that, since our Hamiltonian $H$ is not bounded below, the Littlewood-Paley decomposition associated with $H$ seems to be false for $p \neq 2$ in general. To overcome this difficulty, we consider the following partition of unity on the phase space $\mathbb{R}^{2 d}$ :

$$
\psi_{\varepsilon}(x, \xi)+\chi_{\varepsilon}(x, \xi)=1,
$$

where $\psi_{\varepsilon}$ is supported in $\{(x, \xi) ;\langle x\rangle<\varepsilon|\xi|\}$ for some sufficiently small constant $\varepsilon>0$. It is easy to see that the symbol $p(x, \xi)$ is elliptic on $\operatorname{supp} \psi_{\varepsilon}$ :

$$
C^{-1}|\xi|^{2} \leq p(x, \xi) \leq C|\xi|^{2}, \quad(x, \xi) \in \operatorname{supp} \psi_{\varepsilon}
$$

and we hence can prove a Littlewood-Paley type decomposition of the following form:

$$
\left\|\mathrm{Op}\left(\psi_{\varepsilon}\right) u\right\|_{L^{q}} \leq C_{q}\|u\|_{L^{2}}+C_{q}\left(\sum_{h=2^{-j}, j \geq 0}\left\|\mathrm{Op}_{h}\left(a_{h}\right) f\left(h^{2} H\right) u\right\|_{L^{q}}^{2}\right)^{1 / 2}
$$

where $2 \leq q<\infty,\left\{f\left(h^{2} \cdot\right) ; h=2^{-j}, j \geq 0\right\}$ is a 4 -adic partition of unity on $[1, \infty)$ and $a_{h}$ is an appropriate $h$-dependent symbol supported in $\{|x|<1 / h,|\xi| \in I\}$ for some open interval $I \Subset(0, \infty), \mathrm{Op}\left(\psi_{\varepsilon}\right)$ and $\mathrm{Op}_{h}\left(a_{h}\right)$ denote the corresponding pseudodifferential and semiclassical pseudodifferential operators, respectively.

Then, the idea of the proof of Theorem 1.2 is as follows. In view of the above LittlewoodPaley estimate, the proof is reduced to that of Strichartz estimates for $F(|x|>R) \mathrm{Op}_{h}\left(a_{h}\right) e^{-i t H}$ and $\mathrm{Op}\left(\chi_{\varepsilon}\right) e^{-i t H}$. In order to prove Strichartz estimates for $F(|x|>R) \mathrm{Op}_{h}\left(a_{h}\right) e^{-i t H}$, we use semiclassical approximations of Isozaki-Kitada type. We however note that because of the unboundedness of potentials with respect to $x$, it is difficult to construct directly such approximations. To overcome this difficulty, we introduce a modified Hamiltonian $\widetilde{H}$ due to [37] so that $\widetilde{H}=H$ for $|x| \leq L / h$ and $\widetilde{H}=K$ for $|x| \geq 2 L / h$ for some constant $L \geq 1$. Then, $\widetilde{H}^{h}=h^{2} \widetilde{H}$ can be regarded as a "long-range perturbation" of the semiclassical free Schrödinger operator $H_{0}^{h}=h^{2} H_{0}$. We also introduce the corresponding modified symbol $\widetilde{p}_{h}(x, \xi)$ so that $\widetilde{p}_{h}(x, \xi)=p_{h}(x, \xi)$ for $|x| \leq L / h$ and $\widetilde{p}_{h}(x, \xi)=k(x, \xi)$ for $|x| \geq 2 L / h$. Let $a_{h}^{ \pm}$be supported in outgoing and incoming regions $\{R<|x|<1 / h,|\xi| \in I, \pm \hat{x} \cdot \hat{\xi}>1 / 2\}$, respectively, so that $F(|x|>R) a_{h}=a_{h}^{+}+a_{h}^{-}$, where $\hat{x}=x /|x|$. Rescaling $t \mapsto t h$, we first construct the semiclassical approximations for $e^{-i t \widetilde{H}^{h} / h} \mathrm{Op}_{h}\left(a_{h}^{ \pm}\right)^{*}$ of the following forms

$$
e^{-i t \widetilde{H}^{h} / h} \mathrm{Op}_{h}\left(a_{h}^{ \pm}\right)^{*}=J_{h}\left(S_{h}^{ \pm}, b_{h}^{ \pm}\right) e^{-i t H_{0}^{h} / h} J_{h}\left(S_{h}^{ \pm}, c_{h}^{ \pm}\right)^{*}+O\left(h^{N}\right), \quad 0 \leq \pm t \leq 1 / h,
$$


respectively, where $S_{h}^{ \pm}$solve the Eikonal equation associated to $\widetilde{p}_{h}$ and $J_{h}\left(S_{h}^{ \pm}, b_{h}^{ \pm}\right)$and $J_{h}\left(S_{h}^{ \pm}, c_{h}^{ \pm}\right)$ are associated semiclassical Fourier integral operators. The method of the construction is similar to as that of Robert [27]. On the other hand, we will see that if $L \geq 1$ is large enough, then the Hamilton flow generated by $\widetilde{p}_{h}$ with initial conditions in $\operatorname{supp} a_{h}^{ \pm}$cannot escape from $\{|x| \leq L / h\}$ for $0< \pm t \leq 1 / h$, respectively, i.e.,

$$
\pi_{x}\left(\exp t H_{\widetilde{p}_{h}}\left(\operatorname{supp} a_{h}^{ \pm}\right)\right) \subset\{|x| \leq L / h\}, \quad 0< \pm t \leq 1 / h .
$$

Since $\widetilde{p}_{h}=p_{h}$ for $|x| \leq L / h$, we have

$$
\exp t H_{\widetilde{p}_{h}}\left(\operatorname{supp} a_{h}^{ \pm}\right)=\exp t H_{p_{h}}\left(\operatorname{supp} a_{h}^{ \pm}\right), \quad 0< \pm t \leq 1 / h .
$$

We thus can expect (at least formally) that the corresponding two quantum evolutions are approximately equivalent modulo some smoothing operator. We will prove the following rigorous justification of this formal consideration:

$$
\left\|\left(e^{-i t H^{h} / h}-e^{-i t \widetilde{H}^{h} / h}\right) \operatorname{Op}_{h}\left(a_{h}^{ \pm}\right)^{*}\right\|_{L^{2} \rightarrow L^{2}} \leq C_{M} h^{M}, \quad 0 \leq \pm t \leq 1 / h, M \geq 0,
$$

where $H^{h}=h^{2} H$. By using such approximations for $e^{-i t H^{h} / h} \mathrm{Op}_{h}\left(a_{h}^{ \pm}\right)^{*}$, we prove local-in-time dispersive estimates for $\mathrm{Op}_{h}\left(a_{h}^{ \pm}\right) e^{-i t H} \mathrm{Op}_{h}\left(a_{h}^{ \pm}\right)^{*}$ :

$$
\left\|\mathrm{Op}_{h}\left(a_{h}^{ \pm}\right) e^{-i t H} \mathrm{Op}_{h}\left(a_{h}^{ \pm}\right)^{*}\right\|_{L^{1} \rightarrow L^{\infty}} \leq C|t|^{-d / 2}, \quad 0<h \ll 1,0<|t|<1 .
$$

Strichartz estimates follow from these estimates and the abstract Theorem due to Keel-Tao [19].

Strichartz estimates for $\mathrm{Op}\left(\chi_{\varepsilon}\right) e^{-i t H}$ follow from the following short-time dispersive estimate:

$$
\left\|\mathrm{Op}\left(\chi_{\varepsilon}\right) e^{-i t H} \mathrm{Op}\left(\chi_{\varepsilon}\right)^{*}\right\|_{L^{1} \rightarrow L^{\infty}} \leq C_{\varepsilon}|t|^{-d / 2}, \quad 0<|t|<t_{\varepsilon} \ll 1 .
$$

To prove this, we first construct an approximation for $e^{-i t H} \mathrm{Op}\left(\chi_{\varepsilon}\right)^{*}$ of the following form:

$$
e^{-i t H} \operatorname{Op}\left(\chi_{\varepsilon}\right)^{*}=J(\Psi, a)+O_{H^{-\gamma} \rightarrow H^{\gamma}}(1), \quad|t|<t_{\varepsilon}, \gamma>d / 2,
$$

where the phase function $\Psi=\Psi(t, x, \xi)$ is a solution to a time-dependent Hamilton-Jacobi equation associated to $p(x, \xi)$ and $J(\Psi, a)$ is the corresponding Fourier integral operator. In the construction, the following fact plays an important rule:

$$
\left|\partial_{x}^{\alpha} \partial_{\xi}^{\beta} p(x, \xi)\right| \leq C_{\alpha \beta}, \quad(x, \xi) \in \operatorname{supp} \chi_{\varepsilon},|\alpha+\beta| \geq 2 .
$$

We note that if $\left(g^{j k}\right)_{j k}-\mathrm{Id}_{d} \neq 0$ depends on $x$ then these bounds do not hold without such a restriction of the support. Using these bounds, we construct the phase function $\Psi(t, x, \xi)$ such that

$$
\left|\partial_{x}^{\alpha} \partial_{\xi}^{\beta}(\Psi(t, x, \xi)-x \cdot \xi+p(x, \xi))\right| \leq C_{\alpha \beta}|t|^{2}\langle x\rangle^{2-|\alpha+\beta|} .
$$

Then, we can follow a classical argument (due to [20]) and construct the FIO $J(\Psi, a)$. By the composition formula, $\operatorname{Op}\left(\chi_{\varepsilon}\right) J(\Psi, a)$ is also a FIO and dispersive estimates for this operator follow from the standard stationary phase method. Finally, using an Egorov type lemma, we prove that the remainder, $\operatorname{Op}\left(\chi_{\varepsilon}\right)\left(e^{-i t H} \mathrm{Op}\left(\chi_{\varepsilon}\right)^{*}-J(\Psi, a)\right)$, has a smooth kernel for sufficiently small $t$. 
The proof of Theorem [1.5] is based on a standard idea by [30], see also [6, 4]. Strichartz estimates with loss of derivatives $\langle H\rangle^{1 /(2 p)}$ follow from semiclassical Strichartz estimates up to time scales of order $h$, which can be verified by the standard argument. Moreover, under the nontrapping condition, we will prove that the missing $1 / p$ derivative loss can be recovered by using local smoothing effects due to Doi [11].

The proof of Theorem 1.6 is based on a slight modification of that of Theorem 1.5. By virtue of the Strichartz estimates for $\operatorname{Op}\left(\chi_{\varepsilon}\right) e^{-i t H}$ and the Littlewood-Paley decomposition, it suffices to show

$$
\left\|\mathrm{Op}_{h}\left(a_{h}\right) e^{-i t H} \varphi\right\|_{L^{p}\left([-T, T] ; L^{q}\right)} \leq C_{T} h^{-\varepsilon}\|\varphi\|_{L^{2}}, \quad 0<h \ll 1 .
$$

To prove this estimate, we first prove semiclassical Strichartz estimates for $e^{-i t H} \mathrm{Op}_{h}\left(a_{h}\right)^{*}$ up to time scales of order $h R$, where $R=\inf \left|\pi_{x}\left(\operatorname{supp} a_{h}\right)\right|$. The proof is based on a refinement of the standard WKB approximation for the semiclassical propagator $e^{-i t H^{h} / h} \mathrm{Op}_{h}\left(a_{h}\right)^{*}$. Combining semiclassical Strichartz estimates with a partition of unity argument with respect to $x$, we will obtain the following Strichartz estimate with an inhomogeneous error term:

$$
\left\|\mathrm{Op}_{h}\left(a_{h}\right) e^{-i t H} \varphi\right\|_{L^{p}\left([-T, T] ; L^{q}\right)} \leq C_{T}\|\varphi\|_{L^{2}}+C\left\|\langle x\rangle^{-1 / 2-\varepsilon} h^{-1 / 2-\varepsilon} \mathrm{Op}_{h}\left(a_{h}\right) e^{-i t H} \varphi\right\|_{L^{2}\left([-T, T] ; L^{2}\right)},
$$

for any $\varepsilon>0$, which, combined with local smoothing effects, implies Theorem 1.6.

The paper is organized as follows. We first record some known results on the semiclassical pseudodifferential calculus and prove the above Littlewood-Paley decomposition in Section 2 , Using dispersive estimates, which will be studied in Sections 4 and 5, we shall prove Theorem 1.2 in Section 3. We construct approximations of Isozaki-Kitada type and prove dispersive estimates for $\mathrm{Op}_{h}\left(a_{h}^{ \pm}\right) e^{-i t H} \mathrm{Op}_{h}\left(a_{h}^{ \pm}\right)^{*}$ in Section 4. Section 5 discuss the dispersive estimates for $\operatorname{Op}\left(\chi_{\varepsilon}\right) e^{-i t H} \mathrm{Op}\left(\chi_{\varepsilon}\right)^{*}$. The proof of Theorem [1.5] and Theorem 1.6 are given in Section [6 and Section 7 , respectively.

Acknowledgements. The author would like to express his sincere thanks to Professor Erik Skibsted for valuable discussions and for hospitality at Institut for Matematiske Fag, Aarhus Universitet, where a part of this work was carried out. He also would like to thank the referee for very careful reading the manuscript and for providing valuable suggestions, which substantially helped improving the quality of the paper.

\section{Semiclassical functional calculus}

Throughout this section we assume Assumption 1.1 with $\mu \geq 0$, i.e.,

$$
\left|\partial_{x}^{\alpha} g^{j k}(x)\right|+\langle x\rangle^{-1}\left|\partial_{x}^{\alpha} A_{j}(x)\right|+\langle x\rangle^{-2}\left|\partial_{x}^{\alpha} V(x)\right| \leq C_{\alpha \beta}\langle x\rangle^{-|\alpha|} .
$$

The goal of this section is to prove a Littlewood-Paley type decomposition under a suitable restriction on the initial data. At first we record (without proof) some known results on the pseudodifferential calculus which will be used throughout the paper. We refer to [26, 21] for the details of the proof. 


\subsection{Pseudodifferential calculus}

For the metric $g=d x^{2} /\langle x\rangle^{2}+d \xi^{2} /\langle\xi\rangle^{2}$ and a weight function $m(x, \xi)$ on the phase space $\mathbb{R}^{2 d}$, we use Hörmander's symbol class notation $S(m, g)$, i.e., $a \in S(m, g)$ if and only if $a \in C^{\infty}\left(\mathbb{R}^{2 d}\right)$ and

$$
\left|\partial_{x}^{\alpha} \partial_{\xi}^{\beta} a(x, \xi)\right| \leq C_{\alpha \beta} m(x, \xi)\langle x\rangle^{-|\alpha|}\langle\xi\rangle^{-|\beta|}, \quad \alpha, \beta \in \mathbb{Z}_{+}^{d} .
$$

To a symbol $a \in C^{\infty}\left(\mathbb{R}^{2 d}\right)$ and $h \in(0,1]$, we associate the semiclassical pseudodifferential operator ( $h$-PDO for short) $\mathrm{Op}_{h}(a)$ defined by

$$
\mathrm{Op}_{h}(a) f(x)=\frac{1}{(2 \pi h)^{d}} \int e^{i(x-y) \cdot \xi / h} a(x, \xi) f(y) d y d \xi, \quad f \in \mathcal{S}\left(\mathbb{R}^{d}\right) .
$$

When $h=1$ we write $\operatorname{Op}(a)=\mathrm{Op}_{h}(a)$ for simplicity. The Calderón-Vaillancourt theorem shows that for any symbol $a \in C^{\infty}\left(\mathbb{R}^{2 d}\right)$ satisfying $\left|\partial_{x}^{\alpha} \partial_{\xi}^{\beta} a(x, \xi)\right| \leq C_{\alpha \beta}, \mathrm{Op}_{h}(a)$ is extended to a bounded operator on $L^{2}\left(\mathbb{R}^{d}\right)$ uniformly with respect to $h \in(0,1]$. Moreover, for any symbol $a$ satisfying

$$
\left|\partial_{x}^{\alpha} \partial_{\xi}^{\beta} a(x, \xi)\right| \leq C_{\alpha \beta}\langle\xi\rangle^{-\gamma}, \quad \gamma>d,
$$

$\mathrm{Op}_{h}(a)$ is extended to a bounded operator from $L^{q}\left(\mathbb{R}^{d}\right)$ to $L^{r}\left(\mathbb{R}^{d}\right)$ with the following bounds:

$$
\left\|\mathrm{Op}_{h}(a)\right\|_{L^{q} \rightarrow L^{r}} \leq C_{q r} h^{-d(1 / q-1 / r)}, \quad 1 \leq q \leq r \leq \infty,
$$

where $C_{q r}>0$ is independent of $h \in(0,1]$. These bounds follow from the Schur lemma and an interpolation (see, e.g., [4, Proposition 2.4]).

For two symbols $a \in S\left(m_{1}, g\right)$ and $b \in S\left(m_{2}, g\right)$, the composition $\mathrm{Op}_{h}(a) \mathrm{Op}_{h}(b)$ is also a $h$-PDO and written in the form $\mathrm{Op}_{h}(c)=\mathrm{Op}_{h}(a) \mathrm{Op}_{h}(b)$ with a symbol $c \in S\left(m_{1} m_{2}, g\right)$ given by $c(x, \xi)=\left.e^{i h D_{\eta} D_{z}} a(x, \eta) b(z, \xi)\right|_{z=x, \eta=\xi}$. Moreover, $c(x, \xi)$ has the following expansion

$$
c=\sum_{|\alpha|=0}^{N-1} \frac{h^{|\alpha|}}{i^{|\alpha|} \alpha !} \partial_{\xi}^{\alpha} a \cdot \partial_{x}^{\alpha} b+h^{N} r_{N} \text { with } r_{N} \in S\left(\langle x\rangle^{-N}\langle\xi\rangle^{-N} m_{1} m_{2}, g\right) .
$$

The symbol of the adjoint $\mathrm{Op}_{h}(a)^{*}$ is given by $a^{*}(x, \xi)=\left.e^{i h D_{\eta} D_{z}} a(z, \eta)\right|_{z=x, \eta=\xi} \in S\left(m_{1}, g\right)$ which has the expansion

$$
a^{*}=\sum_{|\alpha|=0}^{N-1} \frac{h^{|\alpha|}}{i^{|\alpha|} \alpha !} \partial_{\xi}^{\alpha} \partial_{x}^{\alpha} a+h^{N} r_{N}^{*} \text { with } r_{N}^{*} \in S\left(\langle x\rangle^{-N}\langle\xi\rangle^{-N} m_{1}, g\right)
$$

\subsection{Littlewood-Paley decomposition}

As we mentioned in the outline of the paper, $H$ is not bounded below in general and we hence cannot expect that the Littlewood-Paley decomposition associated with $H$, which is of the form

$$
\|u\|_{L^{q}} \leq C_{q}\|u\|_{L^{2}}+C_{q}\left(\sum_{j=0}^{\infty}\left\|f\left(2^{-2 j} H\right) u\right\|_{L^{q}}^{2}\right)^{1 / 2}
$$

hold if $q \neq 2$. The standard Littlewood-Paley decomposition associated with $H_{0}$ also does not work well in our case, since the commutator of $H$ with the Littlewood-Paley projection 
$f\left(2^{-2 j} H_{0}\right)$ can grow at spatial infinity. To overcome this difficulty, let us introduce an additional localization as follows. Given a parameter $\varepsilon>0$ and a cut-off function $\varphi \in C_{0}^{\infty}\left(\mathbb{R}_{+}\right)$such that $\varphi \equiv 1$ on $[0,1 / 2]$ and $\operatorname{supp} \varphi \subset[0,1]$, we define $\psi_{\varepsilon}(x, \xi)$ by

$$
\psi_{\varepsilon}(x, \xi)=\varphi\left(\frac{\langle x\rangle}{\varepsilon|\xi|}\right) .
$$

It is easy to see that, for each $\varepsilon>0, \psi_{\varepsilon} \in S(1, g)$ and is supported in $\left\{(x, \xi) \in \mathbb{R}^{2 d} ;\langle x\rangle<\varepsilon|\xi|\right\}$. Moreover, for sufficiently small $\varepsilon>0, p(x, \xi)$ is uniformly elliptic on the support of $\psi_{\varepsilon}$ and $\mathrm{Op}\left(\psi_{\varepsilon}\right) H$ thus is essentially bounded below.

In this subsection we prove a Littlewood-Paley type decomposition on the range of $\operatorname{Op}\left(\psi_{\varepsilon}\right)$. We begin with the following proposition which tells us that, for any $f \in C_{0}^{\infty}(\mathbb{R})$ and $h \in(0,1]$, $\mathrm{Op}\left(\psi_{\varepsilon}\right) f\left(h^{2} H\right)$ is well approximated in terms of the $h$-PDO.

Proposition 2.1. There exists $\varepsilon>0$ such that, for any $f \in C_{0}^{\infty}(\mathbb{R})$ with $\operatorname{supp} f \Subset(0, \infty)$, we can construct bounded families $\left\{a_{h, j}\right\}_{h \in(0,1]} \subset \bigcap_{M>0} S\left(\langle x\rangle^{-j}\langle\xi\rangle^{-M}, g\right), j \geq 0$, such that (1) $a_{h, 0}$ is given explicitly by $a_{h, 0}(x, \xi)=\psi_{\varepsilon}(x, \xi / h) f\left(p_{h}(x, \xi)\right)$. Moreover,

$$
\operatorname{supp} a_{h, j} \subset \operatorname{supp} \psi_{\varepsilon}(\cdot, \cdot / h) \cap \operatorname{supp} f\left(p_{h}\right) \subset\left\{(x, \xi) \in \mathbb{R}^{2 d} ;\langle x\rangle<1 / h,|\xi| \in I\right\},
$$

for some relatively compact open interval $I \Subset(0, \infty)$. In particular, we have

$$
\left\|\mathrm{Op}_{h}\left(a_{h, j}\right)\right\|_{L^{q^{\prime}} \rightarrow L^{q}} \leq C_{j q q^{\prime}} h^{-d\left(1 / q^{\prime}-1 / q\right)}, 1 \leq q^{\prime} \leq q \leq \infty,
$$

uniformly in $h \in(0,1]$.

(2) For any integer $N>d+2$, we set $a_{h}=\sum_{j=0}^{N-1} h^{j} a_{h, j}$. Then,

$$
\left\|\mathrm{Op}\left(\psi_{\varepsilon}\right) f\left(h^{2} H\right)-\mathrm{Op}_{h}\left(a_{h}\right)\right\|_{L^{2} \rightarrow L^{q}} \leq C_{q N} h^{2}, \quad 2 \leq q \leq \infty,
$$

uniformly in $h \in(0,1]$.

The following is an immediate consequence of this proposition.

Corollary 2.2. For any $2 \leq q \leq \infty$ and $h \in(0,1], \mathrm{Op}\left(\psi_{\varepsilon}\right) f\left(h^{2} H\right)$ is bounded from $L^{2}\left(\mathbb{R}^{d}\right)$ to $L^{q}\left(\mathbb{R}^{d}\right)$ and satisfies

$$
\left\|\mathrm{Op}\left(\psi_{\varepsilon}\right) f\left(h^{2} H\right)\right\|_{L^{2} \rightarrow L^{q}} \leq C_{q} h^{-d(1 / 2-1 / q)},
$$

where $C_{q}>0$ is independent of $h \in(0,1]$.

For the low energy part we have the following

Lemma 2.3. For any $f_{0} \in C_{0}^{\infty}(\mathbb{R})$ and $2 \leq q \leq \infty$, we have

$$
\left\|\mathrm{Op}\left(\psi_{\varepsilon}\right) f_{0}(H)\right\|_{L^{2} \rightarrow L^{q}} \leq C_{q} .
$$

Remark 2.4. If $V, A \equiv 0$, then Proposition 2.1. Corollary 2.2 and Lemma 2.3 hold without the additional term $\mathrm{Op}\left(\psi_{\varepsilon}\right)$. Moreover, in this case we see that the remainder satisfies

$$
\left\|f\left(h^{2} H\right)-\mathrm{Op}_{h}\left(a_{h}\right)\right\|_{L^{2} \rightarrow L^{q}} \leq C_{q N} h^{N-d(1 / 2-1 / q)} .
$$


We refer to [6] (for the case on compact manifolds without boundary) and to [4] (for the case with metric perturbations on $\mathbb{R}^{d}$ ). For more general cases with Laplace-Beltrami operators on noncompact manifolds with ends, we refer to [2, 1]. Because of this result, we believe Proposition 2.1 is far from sharp. However, the bounds

$$
\left\|\mathrm{Op}\left(\psi_{\varepsilon}\right) f\left(h^{2} H\right)-\mathrm{Op}_{h}\left(a_{h}\right)\right\|_{L^{2} \rightarrow L^{q}} \leq C_{q N} h, 2 \leq q \leq \infty,
$$

are sufficient to obtain our Littlewood-Paley type decomposition (Proposition 2.5). For more details, we refer to Burq-Gérard-Tvzetkov [6, Corollary 2.3].

Proof of Proposition [2.1. We write

$$
\mathrm{Op}\left(\psi_{\varepsilon}\right)=\mathrm{Op}_{h}\left(\psi_{\varepsilon / h}\right), \quad h \in(0,1]
$$

where $\psi_{\varepsilon / h}(x, \xi)=\psi_{\varepsilon}(x, \xi / h)$ satisfies $\operatorname{supp} \psi_{\varepsilon / h} \subset\{h\langle x\rangle<\varepsilon|\xi|\}$ and

$$
\left|\partial_{x}^{\alpha} \partial_{\xi}^{\beta} \psi_{\varepsilon / h}(x, \xi)\right| \leq C_{\alpha \beta \varepsilon} h^{-|\beta|}\langle x\rangle^{-|\alpha|}\langle\xi / h\rangle^{-|\beta|} \leq C_{\alpha \beta \varepsilon}\langle x\rangle^{-|\alpha|}(h+|\xi|)^{-|\beta|} .
$$

We have by using the Helffer-Sjöstrand formula (see, Helffer-Sjöstrand [16]) that

$$
\mathrm{Op}_{h}\left(\psi_{\varepsilon / h}\right) f\left(h^{2} H\right)=-\frac{1}{2 \pi i} \int_{\mathbb{C}} \frac{\partial \widetilde{f}}{\partial \bar{z}}(z) \operatorname{Op}_{h}\left(\psi_{\varepsilon / h}\right)\left(h^{2} H-z\right)^{-1} d z \wedge d \bar{z},
$$

where $\widetilde{f}(z)$ is an almost analytic extension of $f(\lambda)$. Since $f \in C_{0}^{\infty}(\mathbb{R}), \widetilde{f}(z)$ is also compactly supported and satisfies

$$
\partial_{\bar{z}} \widetilde{f}(z)=O\left(|\operatorname{Im} z|^{M}\right)
$$

for any $M>0$. We may assume $|z| \leq C$ on supp $\tilde{f}$ with some $C>0$. In order to use this formula we shall construct a semiclassical approximation of $\mathrm{Op}_{h}\left(\psi_{\varepsilon / h}\right)\left(h^{2} H-z\right)^{-1}$, in terms of the $h$-PDO, for $z \in \mathbb{C} \backslash[0, \infty)$ with $|z| \leq C$. Although the method is based on the standard semiclassical parametrix construction (see, e.g., [26, 6]), we give the detail of the proof since $\psi_{\varepsilon / h}$ is not uniformly bounded in $S(1, g)$ with respect to $h \in(0,1]$.

We first study the symbol of the resolvent $\left(h^{2} H-z\right)^{-1}$. Let $p_{h}$ and $p_{1, h}$ be as in (1.8) so that $h^{2} H=\mathrm{Op}_{h}\left(p_{h}\right)+h \mathrm{Op}_{h}\left(p_{1, h}\right)$. Since

$$
h|A(x)| \lesssim|\xi|, h^{2}|V(x)| \lesssim|\xi|^{2},
$$

on $\operatorname{supp} \psi_{\varepsilon / h}$, we obtain by (1.7) that

$$
\begin{aligned}
\left|\partial_{x}^{\alpha} \partial_{\xi}^{\beta} p_{h}(x, \xi)\right| & \leq C_{\alpha \beta}\langle x\rangle^{-|\alpha|}|\xi|^{2-|\beta|} \quad \text { if }|\beta| \leq 2, \\
\left|\partial_{x}^{\alpha} \partial_{\xi}^{\beta} p_{1, h}(x, \xi)\right| & \leq C_{\alpha \beta}\langle x\rangle^{-1-|\alpha|}|\xi|^{1-|\beta|} \quad \text { if }|\beta| \leq 1,
\end{aligned}
$$

uniformly in $(x, \xi) \in \operatorname{supp} \psi_{\varepsilon / h}$ and $h \in(0,1]$. Moreover, if $\varepsilon>0$ is sufficiently small then the uniform ellipticity of $k$ implies that $p_{h}$ is also uniformly elliptic on $\operatorname{supp} \psi_{\varepsilon / h}$ :

$$
C_{1}^{-2}|\xi|^{2} \leq p_{h}(x, \xi) \leq C_{1}^{2}|\xi|^{2} \quad \text { if } h\langle x\rangle<\varepsilon|\xi|,
$$

with some $C_{1}>0$, which particularly implies

$$
\frac{1}{\left|p_{h}(x, \xi)-z\right|} \lesssim \begin{cases}|\operatorname{Im} z|^{-1} & \text { if }|\xi| \leq 2 C_{2} \\ \langle\xi\rangle^{-2} & \text { if }|\xi| \geq 2 C_{2}\end{cases}
$$


for $(x, \xi) \in \operatorname{supp} \psi_{\varepsilon / h}, z \notin \mathbb{R}$ and $|z| \leq C$, with some $C_{2}>0$.

Let us now consider a sequence of symbols $q_{j}^{h}=q_{j}^{h}(z, x, \xi)$ (depending holomorphically on $z \notin \mathbb{R})$ defined inductively by

$$
\begin{aligned}
q_{0}^{h} & =\frac{\psi_{\varepsilon / h}}{p_{h}-z}, \\
q_{1}^{h} & =-\frac{1}{p_{h}-z}\left(\sum_{|\alpha|=1} i^{-1} \partial_{\xi}^{\alpha} q_{0}^{h} \cdot \partial_{x}^{\alpha} p_{h}+q_{0}^{h} \cdot p_{1, h}\right), \\
q_{j}^{h} & =-\frac{1}{p_{h}-z}\left(\sum_{\substack{|\alpha|+k=j,|\alpha| \geq 1}} \frac{i^{-|\alpha|}}{\alpha !} \partial_{\xi}^{\alpha} q_{k}^{h} \cdot \partial_{x}^{\alpha} p_{h}+\sum_{|\alpha|+k=j-1,} \frac{i^{-|\alpha|}}{\alpha !} \partial_{\xi}^{\alpha} q_{k}^{h} \cdot \partial_{x}^{\alpha} p_{1, h}\right), j \geq 2 .
\end{aligned}
$$

We then learn by (2.5), (2.6) and (2.8) that

$$
\begin{aligned}
\left|\partial_{x}^{\alpha} \partial_{\xi}^{\beta} q_{0}^{h}(z, x, \xi)\right| & \leq C_{\alpha \beta \varepsilon} \begin{cases}\langle x\rangle^{-|\alpha|}(h+|\xi|)^{-|\beta|}|\operatorname{Im} z|^{-1-|\alpha+\beta|} & \text { if }|\xi| \leq 2 C_{2}, \\
\langle x\rangle^{-|\alpha|}\langle\xi\rangle^{-|\beta|-2} & \text { if }|\xi| \geq 2 C_{2},\end{cases} \\
& \leq C_{\alpha \beta \varepsilon}\langle x\rangle^{-|\alpha|}(h+|\xi|)^{-|\beta|}|\operatorname{Im} z|^{-1-|\alpha+\beta|}
\end{aligned}
$$

for $z \notin \mathbb{R}$ with $|z| \leq C$ and $h \in(0,1]$. We similarly obtain by using (2.6), (2.7) and (2.9) that if $h|\xi| \leq 2 C_{2}$ then

$$
\begin{aligned}
\left|\partial_{x}^{\alpha} \partial_{\xi}^{\beta} q_{1}^{h}(z, x, \xi)\right| \leq & C_{\alpha \beta \varepsilon}\left(\langle x\rangle^{-1-|\alpha|}(h+|\xi|)^{-1-|\beta|}|\xi|^{2}|\operatorname{Im} z|^{-3-|\alpha+\beta|}\right. \\
& \left.+\langle x\rangle^{-1-|\alpha|}(h+|\xi|)^{-|\beta|}(h+|\xi|)|\operatorname{Im} z|^{-2-|\alpha+\beta|}\right) \\
\leq & C_{\alpha \beta \varepsilon}(h+|\xi|)^{2}\langle x\rangle^{-1-|\alpha|}(h+|\xi|)^{-1-|\beta|}|\operatorname{Im} z|^{-3-|\alpha+\beta|},
\end{aligned}
$$

for $z \notin \mathbb{R}$ with $|z| \leq C$ and $h \in(0,1]$. Here note that, in this case, $(h+|\xi|)^{-1}$ may have a singularity at $\xi=0$ as $h \rightarrow+0$. In order to prove the remainder estimate, we will remove this singularity by using a rescaling $\xi \mapsto h \xi$ (see the estimates (2.12) $)$. For $h|\xi| \geq 2 C_{2}, q_{1}^{h}$ does not have such a singularity and satisfies

$$
\left|\partial_{x}^{\alpha} \partial_{\xi}^{\beta} q_{1}^{h}(z, x, \xi)\right| \leq C_{\alpha \beta \varepsilon}\langle x\rangle^{-1-|\alpha|}\langle\xi\rangle^{-|\beta|-4}|\xi| \leq C_{\alpha \beta \varepsilon}\langle x\rangle^{-1-|\alpha|}\langle\xi\rangle^{-|\beta|-3} .
$$

uniformly in $z \notin \mathbb{R}$ with $|z| \leq C$ and $h \in(0,1]$. Since $1 \lesssim h+|\xi|$ if $h|\xi| \gtrsim 1$, summarizing these we have

$$
\left|\partial_{x}^{\alpha} \partial_{\xi}^{\beta} q_{1}^{h}(z, x, \xi)\right| \leq C_{\alpha \beta \varepsilon}\langle x\rangle^{-1-|\alpha|}(h+|\xi|)^{1-|\beta|}|\operatorname{Im} z|^{-3-|\alpha+\beta|}, \quad z \notin \mathbb{R},|z| \leq C, h \in(0,1] .
$$

The estimates (2.9) and a direct computation also show that $q_{1}^{h}$ is of the form

$$
q_{1}^{h}=q_{11}^{h}\left(p_{h}-z\right)^{-3}+q_{10}^{h}\left(p_{h}-z\right)^{-2},
$$

where $q_{1 k}^{h}$ are supported in $\operatorname{supp} \psi_{\varepsilon / h}$, independent of $z$ and satisfy

$$
\left|\partial_{x}^{\alpha} \partial_{\xi}^{\beta} q_{1 k}^{h}(x, \xi)\right| \leq C_{\alpha \beta \varepsilon}\langle x\rangle^{-1-|\alpha|}(h+|\xi|)^{-|\beta|}\langle\xi\rangle^{N_{1}(k)}, \quad h \in(0,1],
$$


with some positive integer $N_{1}(k)>0$. For $j \geq 2$, an induction argument yields that

$$
\left|\partial_{x}^{\alpha} \partial_{\xi}^{\beta} q_{j}^{h}(z, x, \xi)\right| \leq C_{\alpha \beta \varepsilon}\langle x\rangle^{-j-|\alpha|}(h+|\xi|)^{2-j-|\beta|}|\operatorname{Im} z|^{-2 j-1-|\alpha+\beta|}, \quad j \geq 2,
$$

for $z \notin \mathbb{R}$ with $|z| \leq C$ and $h \in(0,1]$. It also follows from an induction on $j$ that there exist a sequence of $z$-independent symbols $\left(q_{j k}^{h}\right)_{k=0}^{j}$ supported in $\operatorname{supp} \psi_{\varepsilon / h}$ and satisfying

$$
\left|\partial_{x}^{\alpha} \partial_{\xi}^{\beta} q_{j k}^{h}(x, \xi)\right| \leq C_{\alpha \beta \varepsilon}\langle x\rangle^{-j-|\alpha|}(h+|\xi|)^{-|\beta|}\langle\xi\rangle^{N_{j}(k)}
$$

with some $N_{j}(k)>0$, such that $q_{j}^{h}$ is of the form

$$
q_{j}^{h}=\sum_{k=0}^{j} q_{j k}^{h}\left(p_{h}-z\right)^{-j-k-1} .
$$

Rescaling $\xi \mapsto h \xi$, we learn by (2.9) and (2.10) that

$$
q_{0}^{h}(z, x, h \xi) \in S(1, g), \quad h^{j} q_{j}^{h}(z, x, h \xi) \in S\left(h^{2}\langle x\rangle^{-j}\langle\xi\rangle^{2-j}, g\right),
$$

with uniform bounds in $h$ and polynomially bounds in $|\operatorname{Im} z|^{-1}$. Then, by the construction of $q_{j}^{h}$, the standard symbolic calculus (not in the semiclassical regime) and the fact that

$$
\operatorname{Op}\left(h^{j} q_{j}^{h}(z, x, h \xi)\right)=h^{j} \operatorname{Op}_{h}\left(q_{j}^{h}\right)
$$

we obtain

$$
\mathrm{Op}\left(\psi_{\varepsilon}\right)=\sum_{j=0}^{N-1} h^{j} \mathrm{Op}_{h}\left(q_{j}^{h}\right)\left(h^{2} H-z\right)+h^{2} \mathrm{Op}\left(r_{h, N, z}\right), \quad N \geq 1,
$$

with some $r_{h, N, z} \in S\left(\langle x\rangle^{-N}\langle\xi\rangle^{2-N}, g\right)$ satisfying

$$
\left|\partial_{x}^{\alpha} \partial_{\xi}^{\beta} r_{h, N, z}(x, \xi)\right| \leq C_{\alpha \beta \varepsilon N}\langle x\rangle^{-N-|\alpha|}\langle\xi\rangle^{2-N-|\beta|}|\operatorname{Im} z|^{-2 N-1-|\alpha+\beta|},
$$

where $C_{\alpha \beta \varepsilon N}>0$ may be taken uniformly in $h \in(0,1], z \in \mathbb{C} \backslash \mathbb{R}$ with $|z| \leq C$ and $x, \xi \in \mathbb{R}^{d}$.

We now use the Helffer-Sjöstrand formula to obtain

$$
\mathrm{Op}\left(\psi_{\varepsilon}\right) f\left(h^{2} H\right)=\sum_{j=0}^{N-1} h^{j} \mathrm{Op}_{h}\left(a_{h, j}\right)+h^{2} R(h, N),
$$

where

$$
\begin{aligned}
a_{h, 0}(x, \xi) & =\psi_{\varepsilon / h}(x, \xi)\left(f \circ p_{h}\right)(x, \xi), \\
a_{h, j}(x, \xi) & =\sum_{k=0}^{j} \frac{(-1)^{k+j}}{(k+j) !} q_{j k}^{h}(x, \xi)\left(f^{(j+k)} \circ p_{h}\right)(x, \xi), 1 \leq j \leq N-1, \\
R(h, N) & =-\frac{1}{2 \pi i} \int_{\mathbb{C}} \frac{\partial \widetilde{f}}{\partial \bar{z}}(z) \mathrm{Op}_{h}\left(r_{h, N, z}\right)\left(h^{2} H-z\right)^{-1} d z \wedge d \bar{z} .
\end{aligned}
$$

Since $\operatorname{supp} q_{j k} \subset \operatorname{supp} \psi_{\varepsilon / h} \subset\{h\langle x\rangle<\varepsilon|\xi|\}$ and $p_{h}$ is uniformly elliptic (i.e., $p_{h} \approx|\xi|^{2}$ ) on the latter region, taking $\varepsilon>0$ smaller if necessary we have

$$
a_{h, j} \subset \operatorname{supp} \psi_{\varepsilon / h} \cap \operatorname{supp} f\left(p_{h}\right) \subset\left\{(x, \xi) ;|x|<1 / h, C_{0}^{-1} \leq|\xi| \leq C_{0}\right\}
$$


with some positive constant $C_{0}>0$, which, combined with (2.11), particularly implies $q_{j k}^{h} \in$ $S\left(\langle x\rangle^{j}, g\right)$ since $h+|\xi| \gtrsim\langle\xi\rangle$ on $\operatorname{supp} \psi_{\varepsilon / h} \cap \operatorname{supp} f\left(p_{h}\right) . \quad\left\{a_{h, j}\right\}_{h \in(0,1]}$ is hence bounded in $\bigcap_{M \geq 0} S\left(\langle x\rangle^{-j}\langle\xi\rangle^{-M}, g\right)$. By virtue of (2.2), we also obtain

$$
\left\|\mathrm{Op}_{h}\left(a_{h, j}\right)\right\|_{L^{q^{\prime}} \rightarrow L^{q}} \leq C_{j q q^{\prime}} h^{-d\left(1 / q^{\prime}-1 / q\right)}, \quad h \in(0,1], 1 \leq q^{\prime} \leq q \leq \infty .
$$

Finally, we shall prove the estimate on the remainder $R(h, N)$. If we choose $N>d+2$, then (2.12) and (2.2) (with $h=1$ ) imply

$$
\|\left.\mathrm{Op}\left(r_{h, N, z}\right)\right|_{L^{2} \rightarrow L^{q}} \leq C_{q N}|\operatorname{Im} z|^{-n(N, q)}, \quad 2 \leq q \leq \infty,
$$

with some positive integer $n(N, q) \geq 2 N+1$, where $C_{q N}>0$ is independent of $h$. Using the bounds $\|\left.\left(h^{2} H-z\right)^{-1}\right|_{L^{2} \rightarrow L^{2}} \leq|\operatorname{Im} z|^{-1},\left|\partial_{\bar{z}} \widetilde{f}(z)\right| \leq C_{M}|\operatorname{Im} z|^{M}$ for any $M \geq 0$ and the fact that $\widetilde{f}$ is compactly supported, we conclude that

$$
\begin{aligned}
\|R(h, N)\|_{L^{2} \rightarrow L^{q}} & \leq C_{M} \int_{\operatorname{supp} \widetilde{f}}|\operatorname{Im} z|^{M}\left\|\operatorname{Op}\left(r_{h, N, z}\right)\right\|_{L^{2} \rightarrow L^{q}}\left\|\left(h^{2} H-z\right)^{-1}\right\|_{L^{2} \rightarrow L^{2}} d z \wedge d \bar{z} \\
& \leq C_{M N q} \int_{\operatorname{supp} \widetilde{f}}|\operatorname{Im} z|^{M-n(N, q)-1} d z \wedge d \bar{z} \\
& \leq C_{M N q}
\end{aligned}
$$

provided that $M$ is large enough. We complete the proof.

Proof of Lemma 2.3. By the same argument as above with $h=1$, we can see that

$$
\mathrm{Op}\left(\psi_{\varepsilon}\right) f_{0}(H)=\sum_{j=0}^{N-1} \mathrm{Op}\left(a_{j}\right)+R(N)
$$

where $a_{j} \in \bigcap_{M \geq 0} S\left(\langle x\rangle^{-j}\langle\xi\rangle^{-M}, g\right)$ are supported in

$$
\operatorname{supp} \psi_{\varepsilon} \cap \operatorname{supp} f_{0}(p) \subset\left\{(x, \xi) \in \mathbb{R}^{2 d} ;\langle x\rangle<\varepsilon|\xi|,|\xi| \lesssim 1\right\}
$$

and $R(N)$ satisfies

$$
\|R(h, N)\|_{L^{2} \rightarrow L^{q}} \leq C_{N q}, \quad 2 \leq q \leq \infty,
$$

if $N>d+2$. The assertion then follows from (2.2).

Consider a 4-adic partition of unity:

$$
f_{0}(\lambda)+\sum_{h} f\left(h^{2} \lambda\right)=1, \quad \lambda \in \mathbb{R}
$$

where $f_{0}, f \in C_{0}^{\infty}(\mathbb{R})$ with supp $f_{0} \subset[-1,1]$, supp $f \subset[1 / 4,4]$ and $\sum_{h}$ means that, in the sum, $h$ takes all negative powers of 2 as values, i.e., $\sum_{h}=\sum_{h=2^{-j}, j \geq 0}$. Let $F \in C_{0}^{\infty}(\mathbb{R})$ be such that $\operatorname{supp} F \subset[1 / 8,8]$ and $F \equiv 1$ on $\operatorname{supp} f$. The spectral decomposition theorem implies

$$
1=f_{0}(H)+\sum_{h} f\left(h^{2} H\right)=f_{0}(H)+\sum_{h} F\left(h^{2} H\right) f\left(h^{2} H\right) .
$$

Let $a_{h} \in S(1, g)$ be as in Proposition 2.1 with $f=F$. Using Proposition 2.1, we obtain a Littlewood-Paley type estimates on a range of $\mathrm{Op}\left(\psi_{\varepsilon}\right)$. 
Proposition 2.5. For any $2 \leq q<\infty$,

$$
\left\|\mathrm{Op}\left(\psi_{\varepsilon}\right) u\right\|_{L^{q}\left(\mathbb{R}^{d}\right)} \leq C_{q}\|u\|_{L^{2}\left(\mathbb{R}^{d}\right)}+C_{q}\left(\sum_{h}\left\|\mathrm{Op}_{h}\left(a_{h}\right) f\left(h^{2} H\right) u\right\|_{L^{q}\left(\mathbb{R}^{d}\right)}\right)^{1 / 2} .
$$

Proof. The proof is same as that of [6, Corollary 2.3] and we omit details.

Corollary 2.6. Let $\varepsilon>0$ and $\psi_{\varepsilon}$ be as above and $\chi_{\varepsilon}=1-\psi_{\varepsilon}$. Let $\rho \in C^{\infty}\left(\mathbb{R}^{d}\right)$ be such that

$$
\left|\partial_{x}^{\alpha} \rho(x)\right| \leq C_{\alpha}\langle x\rangle^{-|\alpha|}, \quad \alpha \in \mathbb{Z}_{+}^{d} .
$$

Then, for any $T>0$ and any $(p, q)$ satisfying $p \geq 2, q<\infty$ and $2 / p=d(1 / 2-1 / q)$, there exists $C_{T}>0$ such that

$$
\begin{aligned}
\left\|\rho e^{-i t H} \varphi\right\|_{L^{p}\left([-T, T] ; L^{q}\left(\mathbb{R}^{d}\right)\right)} & \leq C_{T}\|\varphi\|_{L^{2}\left(\mathbb{R}^{d}\right)}+C\left\|\mathrm{Op}\left(\chi_{\varepsilon}\right) e^{-i t H} \varphi\right\|_{L^{p}\left([-T, T] ; L^{q}\left(\mathbb{R}^{d}\right)\right)} \\
& +C\left(\sum_{h}\left\|\mathrm{Op}_{h}\left(a_{h}\right) e^{-i t H} f\left(h^{2} H\right) \varphi\right\|_{L^{p}\left([-T, T] ; L^{q}\left(\mathbb{R}^{d}\right)\right)}^{2}\right)^{1 / 2},
\end{aligned}
$$

where $a_{h}$ is given by Proposition 2.1 with $\psi_{\varepsilon}$ replaced by $\rho \psi_{\varepsilon}$. In particular, $a_{h}(x, \xi)$ is supported in $\operatorname{supp} \rho(x) \psi(x, \xi / h) F\left(p_{h}(x, \xi)\right)$.

Proof. This proposition follows from the $L^{2}$-boundedness of $e^{-i t H}$, Propositions 2.1 and 2.5 (with $\psi_{\varepsilon}$ replaced by $\rho \psi_{\varepsilon}$ ) and the Minkowski inequality.

\section{Proof of Theorem 1.2}

In this section we prove Theorem 1.2 under Assumption 1.1 with $\mu>0$. We first state two key estimates which we will prove in later sections. For $R>0$, an open interval $I \Subset(0, \infty)$ and $\sigma \in(-1,1)$, we define the outgoing and incoming regions $\Gamma^{ \pm}(R, I, \sigma)$ by

$$
\Gamma^{ \pm}(R, I, \sigma):=\left\{(x, \xi) \in \mathbb{R}^{2 d} ;|x|>R,|\xi| \in I, \pm \frac{x \cdot \xi}{|x||\xi|}>-\sigma\right\},
$$

respectively. We then have the following (local-in-time) dispersive estimates:

Proposition 3.1. Suppose that $H$ satisfies Assumption 1.1 with $\mu>0$. Let $I \Subset(0, \infty)$ and $\sigma \in(-1,1)$. Then, for sufficiently large $R \geq 1$, small $h_{0}>0$ and any symbols $a_{h}^{ \pm} \in S(1, g)$ supported in $\Gamma^{ \pm}(R, I, \sigma) \cap\{x ;|x|<1 / h\}$, we have

$$
\left\|\mathrm{Op}_{h}\left(a_{h}^{ \pm}\right) e^{-i t H} \mathrm{Op}_{h}\left(a_{h}^{ \pm}\right)^{*}\right\|_{L^{1} \rightarrow L^{\infty}} \leq C|t|^{-d / 2}, \quad 0<|t| \leq 1,
$$

uniformly with respect to $h \in\left(0, h_{0}\right]$.

We prove this proposition in Section 4, In the region $\{|x| \gtrsim|\xi|\}$, we have the following (short-time) dispersive estimates:

Proposition 3.2. Suppose that $H$ satisfies Assumption 1.1 with $\mu \geq 0$. Let us fix arbitrarily $\varepsilon>0$. Then, there exists $t_{\varepsilon}>0$ such that, for any symbol $\chi_{\varepsilon} \in S(1, g)$ supported in $\{(x, \xi) ;\langle x\rangle \geq$ $\varepsilon|\xi|\}$, we have

$$
\|\left.\mathrm{Op}\left(\chi_{\varepsilon}\right) e^{-i t H} \mathrm{Op}\left(\chi_{\varepsilon}\right)^{*}\right|_{L^{1} \rightarrow L^{\infty}} \leq C_{\varepsilon}|t|^{-d / 2}, \quad 0<|t| \leq t_{\varepsilon}
$$


We prove this proposition in Section 5 .

Proof of Theorem 1.2. Taking $\rho \in C^{\infty}\left(\mathbb{R}^{d}\right)$ so that $0 \leq \rho(x) \leq 1, \rho(x)=1$ for $|x| \geq 1$ and $\rho(x)=0$ for $|x| \leq 1 / 2$, we set $\rho_{R}(x)=\rho(x / R)$. In order to prove Theorem 1.2, it suffices to show

$$
\left\|\rho_{R} e^{-i t H} \varphi\right\|_{L^{p}\left([-T, T] ; L^{q}\left(\mathbb{R}^{d}\right)\right)} \leq C_{T}\|\varphi\|_{L^{2}\left(\mathbb{R}^{d}\right)},
$$

for sufficiently large $R \geq 1$. We also may assume without loss of generality that $T>0$ is sufficiently small. Indeed, if the above estimate holds on $\left[-T_{0}, T_{0}\right]$ with some $T_{0}>0$ then we obtain by the unitarity of $e^{-i t H}$ on $L^{2}$ that, for any $T>T_{0}$,

$$
\begin{aligned}
\left\|\rho_{R} e^{-i t H} \varphi\right\|_{L^{p}\left([-T, T] ; L^{q}\left(\mathbb{R}^{d}\right)\right)}^{p} & \lesssim \sum_{k=-\left[T / T_{0}\right]}^{\left[T / T_{0}\right]+1}\left\|\rho_{R} e^{-i t H} e^{-i(k+1) H} \varphi\right\|_{L^{p}\left(\left[-T_{0}, T_{0}\right] ; L^{q}\left(\mathbb{R}^{d}\right)\right)}^{p} \\
& \lesssim\left(T / T_{0}\right) C_{T_{0}}^{p}\|\varphi\|_{L^{2}\left(\mathbb{R}^{d}\right)^{p}}^{p}
\end{aligned}
$$

Let $a_{h}$ be as in Proposition 2.1. Replacing $\psi_{\varepsilon}$ with $\rho_{R} \psi_{\varepsilon}$ and taking $\varepsilon>0$ smaller if necessary, we may assume without loss of generality that $\operatorname{supp} a_{h} \subset\{(x, \xi) ; R<|x|<1 / h,|\xi| \in I\}$ for some open interval $I \Subset(0, \infty)$. Choosing $\theta^{ \pm} \in C^{\infty}([-1,1])$ so that $\theta^{+}+\theta^{-}=1, \theta^{+}=1$ on $[1 / 2,1]$ and $\theta^{+}=0$ on $[-1,-1 / 2]$, we set $a_{h}^{ \pm}(x, \xi)=a_{h}(x, \xi) \theta^{ \pm}(\hat{x} \cdot \hat{\xi})$, where $\hat{x}=x /|x|$. It is clear that $\left\{a_{h}^{ \pm}\right\}_{h \in(0,1]}$ is bounded in $S(1, g)$ and $\operatorname{supp} a_{h}^{ \pm} \subset \Gamma^{ \pm}(R, I, 1 / 2) \cap\{x ;|x|<1 / h\}$, and that $a_{h}=a_{h}^{+}+a_{h}^{-}$. We now apply Proposition 3.1 to $a_{h}^{ \pm}$and obtain the local-in-time dispersive estimate for $\mathrm{Op}_{h}\left(a_{h}^{ \pm}\right) e^{-i t H} \mathrm{Op}_{h}\left(a_{h}^{ \pm}\right)^{*}$ (uniformly in $h \in\left(0, h_{0}\right]$ ), which, combined with the $L^{2}$ boundedness of $\mathrm{Op}_{h}\left(a_{h}^{ \pm}\right) e^{-i t H}$ and the abstract Theorem due to Keel-Tao [19], implies Strichartz estimates for $\mathrm{Op}_{h}\left(a_{h}\right) e^{-i t H}$ :

$$
\begin{aligned}
\left\|\mathrm{Op}_{h}\left(a_{h}\right) e^{-i t H} \varphi\right\|_{L^{p}\left([-1,1] ; L^{q}\left(\mathbb{R}^{d}\right)\right)} & \leq \sum_{ \pm}\left\|\mathrm{Op}_{h}\left(a_{h}^{ \pm}\right) e^{-i t H} \varphi\right\|_{L^{p}\left([-1,1] ; L^{q}\left(\mathbb{R}^{d}\right)\right)} \\
& \leq C\|\varphi\|_{L^{2}\left(\mathbb{R}^{d}\right)}
\end{aligned}
$$

uniformly with respect to $h \in\left(0, h_{0}\right]$. Since $\mathrm{Op}_{h}\left(a_{h}\right)$ is bounded from $L^{2}\left(\mathbb{R}^{d}\right)$ to $L^{q}\left(\mathbb{R}^{d}\right)$ with the bound of order $O\left(h^{-d(1 / 2-1 / q)}\right)$, for $h_{0}<h \leq 1$ we have

$$
\sum_{h_{0}<h \leq 1}\left\|\mathrm{Op}_{h}\left(a_{h}\right) e^{-i t H} f\left(h^{2} H\right) \varphi\right\|_{L^{p}\left([-1,1] ; L^{q}\left(\mathbb{R}^{d}\right)\right)}^{2} \leq C\left(h_{0}\right)\|\varphi\|_{L^{2}\left(\mathbb{R}^{d}\right)}^{2} .
$$

with some $C\left(h_{0}\right)>0$. Using these two bounds, we obtain

$$
\begin{aligned}
& \sum_{h}\left\|\mathrm{Op}_{h}\left(a_{h}\right) e^{-i t H} f\left(h^{2} H\right) \varphi\right\|_{L^{p}\left([-1,1] ; L^{q}\left(\mathbb{R}^{d}\right)\right)}^{2} \\
& \leq C \sum_{0<h<h_{0}}\left\|f\left(h^{2} H\right) \varphi\right\|_{\left.L^{2}\left(\mathbb{R}^{d}\right)\right)}^{2}+C\left(h_{0}\right)\|\varphi\|_{L^{2}\left(\mathbb{R}^{d}\right)}^{2} \\
& \leq C\|\varphi\|_{L^{2}\left(\mathbb{R}^{d}\right)}^{2} .
\end{aligned}
$$

On the other hand, Strichartz estimates for $\mathrm{Op}\left(\chi_{\varepsilon}\right) e^{-i t H}$ is an immediate consequence of Proposition 3.2, By virtue of Corollary 2.6, we complete the proof. 


\section{Semiclassical approximations for outgoing propagators}

Throughout this section we assume Assumption 1.1 with $\mu>0$. We here study the behavior of $e^{-i t H} \mathrm{Op}_{h}\left(a_{h}^{ \pm}\right)^{*}$, where $a_{h}^{ \pm} \in S(1, g)$ are supported in $\Gamma^{ \pm}(R, I, \sigma) \cap\{|x|<1 / h\}$, respectively. The main goal of this section is to prove Proposition 3.1. For simplicity, we consider the outgoing propagator $e^{-i t H} \mathrm{Op}_{h}\left(a_{h}^{+}\right)^{*}$ for $0 \leq t \leq 1$ only, and the proof for the incoming case is analogous.

In order to prove dispersive estimates, we construct a semiclassical approximation for the outgoing propagator $e^{-i t H} \mathrm{Op}_{h}\left(a_{h}^{+}\right)^{*}$ by using the method of Isozaki-Kitada. Namely, rescaling $t \mapsto t h$ and setting $H^{h}=h^{2} H, H_{0}^{h}=-h^{2} \Delta / 2$, we consider an approximation for the semiclassical propagator $e^{-i t H^{h} / h} \mathrm{Op}_{h}\left(a_{h}^{+}\right)^{*}$ of the following form

$$
e^{-i t H^{h} / h} \mathrm{Op}_{h}\left(a_{h}^{+}\right)^{*}=J_{h}\left(S_{h}^{+}, b_{h}^{+}\right) e^{-i t H_{0}^{h} / h} J_{h}\left(S_{h}^{+}, c_{h}^{+}\right)^{*}+O\left(h^{N}\right), \quad 0 \leq t \leq h^{-1},
$$

where $S_{h}^{+}$solves a suitable Eikonal equation in the outgoing region and $J\left(S_{h}^{+}, w\right)$ is the corresponding semiclassical Fourier integral operator ( $h$-FIO for short):

$$
J_{h}\left(S_{h}^{+}, w\right) f(x)=(2 \pi h)^{-d} \int e^{i\left(S_{h}^{+}(x, \xi)-y \cdot \xi\right) / h} w(x, \xi) f(y) d y d \xi
$$

Such approximations (uniformly in time) have been studied by [28] for Schrödinger operators with long-range potentials, and by [26, 27, 4, for the case of long-range metric perturbations. We also refer to the original paper by Isozaki-Kitada [17] in which the existence and asymptotic completeness of modified wave operators (with time-independent modifiers) were established for the case of Schrödinger operators with long-range potentials. We note that, in these cases, we do not need the additional restriction of the initial data in $\{|x|<1 / h\}$. On the other hand, in a recent paper [24], we constructed such approximations (locally in time) for the case with long-range metric perturbations, combined with potentials growing subquadratically at infinity, under the additional restriction on the initial data into $\{|x|<1 / h\}$.

As we mentioned in the outline of the paper, we first construct an approximation for the modified propagator $e^{-i t \widetilde{H}^{h} / h}$, where $\widetilde{H}^{h}$ is defined as follows. Taking arbitrarily a cut-off function $\psi \in C_{0}^{\infty}\left(\mathbb{R}^{d}\right)$ such that $0 \leq \psi \leq 1, \psi \equiv 1$ for $|x| \leq 1 / 2$ and $\psi \equiv 0$ for $|x| \geq 1$, we define truncated electric and magnetic potentials, $V_{h}$ and $A_{h}=\left(A_{h, j}\right)_{j}$ by $V_{h}(x):=\psi(h x / L) V(x)$, $A_{h, j}(x)=\psi(h x / L) A_{j}(x)$, respectively. It is easy to see that

$$
V_{h} \equiv V, A_{h, j} \equiv A_{j} \text { on }\{|x| \leq L /(2 h)\}, \quad \operatorname{supp} A_{h, j}, \operatorname{supp} V_{h} \subset\{|x| \leq L / h\},
$$

and that, for any $\alpha \in \mathbb{Z}_{+}^{d}$ there exists $C_{L, \alpha}>0$, independent of $x, h$, such that

$$
h^{2}\left|\partial_{x}^{\alpha} V_{h}(x)\right|+h\left|\partial_{x}^{\alpha} A_{h}(x)\right| \leq C_{\alpha, L}\langle x\rangle^{-\mu-|\alpha|} .
$$

Let us define $\widetilde{H}^{h}$ by

$$
\widetilde{H}^{h}=\frac{1}{2} \sum_{j, k=1}^{d}\left(-i h \partial_{j}-h A_{h, j}(x)\right) g^{j k}(x)\left(-i h \partial_{k}-h A_{h, k}(x)\right)+h^{2} V_{h}(x) .
$$

We consider $\widetilde{H}^{h}$ as a "semiclassical" Schrödinger operator with $h$-dependent electromagnetic potentials $h^{2} V_{h}$ and $h A_{h}$. By virtue of the estimates on $g^{j k}, A_{h}$ and $V_{h}, \widetilde{H}^{h}$ can be regarded as 
a long-range perturbation of the semiclassical free Schrödinger operator $H_{0}^{h}=-h^{2} \Delta / 2$. Such a type modification has been used to prove Strichartz estimates and local smoothing effects (with loss of derivatives) for Schrödinger equations with superquadratic potentials (see, Yajima-Zhang [37, Section 4]). Let us denote by $\widetilde{p}_{h}$ the corresponding modified symbol:

$$
\widetilde{p}_{h}(x, \xi)=\frac{1}{2} \sum_{j, k=1}^{d} g^{j k}(x)\left(\xi_{j}-h A_{h, j}(x)\right)\left(\xi_{k}-h A_{h, k}(x)\right)+h^{2} V_{h}(x) .
$$

The following proposition, which was proved by [27], provides the existence of the phase function of $h$-FIO's.

Proposition 4.1. Let us fix an open interval $I \Subset(0, \infty),-1<\sigma<1$ and $L>0$. Then, there exist $R_{0}, h_{0}>0$ and a family of smooth and real-valued functions

$$
\left\{S_{h}^{+} ; 0<h \leq h_{0}, R \geq R_{0}\right\} \subset C^{\infty}\left(\mathbb{R}^{2 d} ; \mathbb{R}\right)
$$

satisfying the Eikonal equation associated to $\widetilde{p}_{h}$ :

$$
\widetilde{p}_{h}\left(x, \partial_{x} S_{h}^{+}(x, \xi)\right)=|\xi|^{2} / 2, \quad(x, \xi) \in \Gamma^{+}(R, I, \sigma),
$$

such that

$$
\left|S_{h}^{+}(x, \xi)-x \cdot \xi\right| \leq C\langle x\rangle^{1-\mu} \quad, x, \xi \in \mathbb{R}^{d}
$$

Moreover, for any $|\alpha+\beta| \geq 1$,

$$
\left|\partial_{x}^{\alpha} \partial_{\xi}^{\beta}\left(S_{h}^{+}(x, \xi)-x \cdot \xi\right)\right| \leq C_{\alpha \beta} \min \left\{R^{1-\mu-|\alpha|},\langle x\rangle^{1-\mu-|\alpha|}\right\}, \quad x, \xi \in \mathbb{R}^{d} .
$$

Here $C, C_{\alpha \beta}>0$ are independent of $x, \xi, R$ and $h$.

Proof. Since $h^{2} V_{h}$ and $h A_{h}$ are of long-range type uniformly with respect to $h \in(0,1]$ (the constant $C_{L, \alpha}$ in (4.1) can be taken independently of $h$ ), the proof is same as that of [27, Proposition 4.1] and we omit it. For the $R$ dependence, we refer to [4, Proposition 3.1].

Remark 4.2. The crucial point to obtain the estimates (4.4) and (4.5) is the uniform bound (4.1) and we do not have to use the support properties of $A_{h}$ and $V_{h}$. Suppose that $A$ and $V$ satisfy Assumption 1.1 with $\mu \geq 0$, i.e., $\langle x\rangle^{-1}\left|\partial_{x}^{\alpha} A(x)\right|+\langle x\rangle^{-2}\left|\partial_{x}^{\alpha} V(x)\right| \leq C_{\alpha \beta}\langle x\rangle^{-|\alpha|}$. If $g^{j k}$ is of long-range type, then we still can construct the solution $S_{h}^{+}$to (4.3) by using the support properties of $A_{h}$ and $V_{h}$, provided that if $L>0$, being independent of $h$, is small enough. However, in this case, $S_{h}^{+}-x \cdot \xi$ behaves like $\langle x\rangle^{1-\mu} h^{-1}$ as $h \rightarrow 0$, and we cannot obtain the uniform $L^{2}$-boundedness of the corresponding $h$-FIO. This is one of the reason why we exclude the critical case $\mu=0$.

To the phase $S_{h}^{+}$and an amplitude $a \in S(1, g)$, we associate the $h$-FIO defined by

$$
J_{h}\left(S_{h}^{+}, a\right) f(x)=(2 \pi h)^{-d} \int e^{i\left(S_{h}^{+}(x, \xi)-y \cdot \xi\right) / h} a(x, \xi) f(y) d y d \xi .
$$


Using (4.5), for sufficiently large $R>0$, we have

$$
\begin{aligned}
\left|\partial_{\xi} \otimes \partial_{x} S_{h}^{+}(x, \xi)-\mathrm{Id}\right| & \leq C\langle R\rangle^{-\mu}<1 / 2, \\
\left|\partial_{x}^{\alpha} \partial_{\xi}^{\beta} S_{h}^{+}(x, \xi)\right| & \leq C_{\alpha \beta} \text { for }|\alpha+\beta| \geq 2,
\end{aligned}
$$

uniformly in $h \in\left(0, h_{0}\right]$. Therefore, the standard $L^{2}$-boundedness of FIO implies that $J_{h}\left(S_{h}^{+}, a\right)$ is uniformly bounded on $L^{2}\left(\mathbb{R}^{d}\right)$ with respect to $h \in\left(0, h_{0}\right]$.

We now construct the outgoing approximation for $e^{-i t \widetilde{H}^{h} / h}$.

Theorem 4.3. Let us fix arbitrarily open intervals $I \Subset I_{0} \Subset I_{1} \Subset I_{2} \Subset(0, \infty),-1<\sigma<$ $\sigma_{0}<\sigma_{1}<\sigma_{2}<1$ and $L>0$. Let $R_{0}$ and $h_{0}$ be as in Proposition 4.1 with $I, \sigma$ replaced by $I_{2}, \sigma_{2}$, respectively. Then, for every integer $N \geq 0$, the followings hold uniformly with respect to $R \geq R_{0}$ and $h \in\left(0, h_{0}\right]$.

(1) There exists a symbol

$$
b_{h}^{+}=\sum_{j=0}^{N-1} h^{j} b_{h, j}^{+} \quad \text { with } \quad b_{h, j}^{+} \in S\left(\langle x\rangle^{-j}\langle\xi\rangle^{-j}, g\right), \operatorname{supp} b_{h, j}^{+} \subset \Gamma^{+}\left(R^{1 / 3}, I_{1}, \sigma_{1}\right),
$$

such that, for any $a^{+} \in S(1, g)$ with $\operatorname{supp} a^{+} \subset \Gamma^{+}(R, I, \sigma)$, we can find

$$
c_{h}^{+}=\sum_{j=0}^{N-1} h^{j} c_{h, j}^{+} \quad \text { with } \quad c_{h, j}^{+} \in S\left(\langle x\rangle^{-j}\langle\xi\rangle^{-j}, g\right), \operatorname{supp} c_{h, j}^{+} \subset \Gamma^{+}\left(R^{1 / 2}, I_{0}, \sigma_{0}\right),
$$

such that, for all $0 \leq t \leq h^{-1}, e^{-i t \widetilde{H}^{h} / h} \mathrm{Op}_{h}\left(a^{+}\right)^{*}$ can be brought to the form

$$
e^{-i t \widetilde{H}^{h} / h} \operatorname{Op}_{h}\left(a^{+}\right)^{*}=J_{h}\left(S_{h}^{+}, b_{h}^{+}\right) e^{-i t H_{0}^{h} / h} J_{h}\left(S_{h}^{+}, c_{h}^{+}\right)^{*}+Q_{\mathrm{IK}}^{+}(t, h, N),
$$

where $J_{h}\left(S_{h}^{+}, w\right), w=b_{h}^{+}, c_{h}^{+}$, are $h$-FIO's associated to the phase $S_{h}^{+}$defined in Proposition 4.1 with $R, I$ and $\sigma$ replaced by $R^{1 / 4}, I_{2}, \sigma_{2}$, respectively. Moreover, for any integer $s \geq 0$ with $2 s \leq N-1$, the remainder $Q_{\mathrm{IK}}^{+}(t, h, N)$ satisfies

$$
\left\|\langle D\rangle^{s} Q_{\mathrm{IK}}^{+}(t, h, N)\langle D\rangle^{s}\right\|_{L^{2} \rightarrow L^{2}} \leq C_{N s} h^{N-2 s-1},
$$

uniformly with respect to $h \in\left(0, h_{0}\right]$ and $0 \leq t \leq h^{-1}$.

(2) Let $K_{S_{h}^{+}}(t, x, y)$ be the distribution kernel of $J_{h}\left(S_{h}^{+}, b_{h}^{+}\right) e^{-i t H_{0}^{h} / h} J_{h}\left(S_{h}^{+}, c_{h}^{+}\right)^{*}$. Then, $K_{S_{h}^{+}}$ satisfies dispersive estimates:

$$
\left|K_{S_{h}^{+}}(t, x, y)\right| \leq C|t h|^{-d / 2},
$$

uniformly with respect to $h \in\left(0, h_{0}\right], x, y \in \mathbb{R}^{d}$ and $0 \leq t \leq h^{-1}$.

Proof. This theorem is basically known and we hence omit the proof. For the construction of the amplitudes $b_{h}^{+}$and $c_{h}^{+}$, we refer to Robert [27, Section 4] (see also Bouclet-Tzvetkov [4, Section 3]). The remainder estimate (4.6) can be proved by the same argument as that in [4, Proposition 3.3, Lemma 3.4] combined with the following simple estimates:

$$
\left\|\langle D\rangle^{s}\left(\widetilde{H}^{h}+C_{1}\right)^{-s / 2}\right\|_{L^{2} \rightarrow L^{2}} \leq C_{s} h^{-s}, \quad s \geq 0 .
$$


where $C_{1}>0$ is a large constant. Note that these estimates follow from the obvious bounds

$$
\left\|\langle D\rangle^{s}\langle h D\rangle^{-s}\right\|_{L^{2} \rightarrow L^{2}} \leq C_{s} h^{-s}, \quad s \geq 0
$$

and the fact that $\left(\widetilde{p}_{h}+h \widetilde{p}_{1, h}+C_{1}\right)^{-s / 2} \in S\left(\langle\xi\rangle^{-s}, g\right)$ since $\widetilde{p}_{h}+h \widetilde{p}_{1, h}+C_{1}$ is uniformly elliptic for sufficiently large $C_{1}>0$. The dispersive estimate (4.7) can be verified by the same argument as that in [4, Lemma 4.4].

The following lemma, which has been essentially proved by [24, tells us that one can still construct the semiclassical approximation for the original propagator $e^{-i t H^{h} / h}$ if we restrict the support of initial data in the region $\Gamma^{+}(R, J, \sigma) \cap\left\{x ;|x|<h^{-1}\right\}$.

Lemma 4.4. Suppose that $\left\{a_{h}^{+}\right\}_{h \in(0,1]}$ is a bounded set in $S(1, g)$ with symbols supported in $\Gamma^{+}(R, I, \sigma) \cap\left\{x ;|x|<h^{-1}\right\}$. Then, there exists $L>1$ such that, for any $M, s \geq 0, h \in\left(0, h_{0}\right]$ and $0 \leq t \leq h^{-1}$, we have

$$
\left\|\left(e^{-i t H^{h} / h}-e^{-i t \widetilde{H}^{h} / h}\right) \operatorname{Op}_{h}\left(a_{h}^{+}\right)^{*}\langle D\rangle^{s}\right\|_{L^{2} \rightarrow L^{2}} \leq C_{M, s} h^{M-s},
$$

where $C_{M, s}>0$ is independent of $h$ and $t$.

In order to prove this lemma, we need the following.

Lemma 4.5. Let $f_{h} \in C^{\infty}\left(\mathbb{R}^{d}\right)$ be such that for any $\alpha \in \mathbb{Z}_{+}^{d}$,

$$
\left|\partial_{x}^{\alpha} f_{h}(x)\right| \leq C_{\alpha}
$$

uniformly with respect to $h \in\left(0, h_{0}\right]$ and such that $\operatorname{supp} f_{h} \subset\{|x| \geq L /(2 h)\}$. Let $L>1$ be large enough. Then, under the conditions in Lemma 4.4, we have

$$
\left\|f_{h}(x)\langle D\rangle^{\gamma} e^{-i t \widetilde{H}^{h} / h} \mathrm{Op}_{h}\left(a_{h}^{+}\right)^{*}\langle D\rangle^{s}\right\|_{L^{2} \rightarrow L^{2}} \leq C_{M, s, \gamma} h^{M-s-\gamma},
$$

for any $s, \gamma \geq 0$ and $M \geq 0$, uniformly with respect $h \in\left(0, h_{0}\right]$ and $0 \leq t \leq 1 / h$.

Proof. We apply Theorem 4.3 to $e^{-i t \widetilde{H}^{h} / h} \mathrm{Op}_{h}\left(a_{h}^{+}\right)^{*}$ and obtain

$$
e^{-i t \widetilde{H}^{h} / h} \operatorname{Op}_{h}\left(a_{h}^{+}\right)^{*}=J_{h}\left(S_{h}^{+}, b_{h}^{+}\right) e^{-i t H_{0}^{h} / h} J_{h}\left(S_{h}^{+}, c_{h}^{+}\right)^{*}+Q_{\mathrm{IK}}^{+}(t, h, N) .
$$

By virtue of (4.6), the remainder $f_{h}(x)\langle D\rangle^{\gamma} Q_{\mathrm{IK}}^{+}(t, h, N)\langle D\rangle^{s}$ is bounded on $L^{2}\left(\mathbb{R}^{d}\right)$ with the norm dominated by $C_{N s \gamma} h^{N-\gamma-s-1}$, uniformly with respect $h \in\left(0, h_{0}\right]$ and $t \in[0,1 / h]$. On the other hand, by virtue of (4.5), the phase of $K_{S_{h}^{+}}(t, x, y)$, which is given by

$$
\Phi_{h}^{+}(t, x, y, \xi)=S_{h}^{+}(x, \xi)-\frac{1}{2} t|\xi|^{2}-S_{h}^{+}(y, \xi),
$$

satisfies $\partial_{\xi} \Phi_{h}^{+}(t, x, y, \xi)=(x-y)\left(\operatorname{Id}+O\left(R^{-\mu / 4}\right)\right)-t \xi$. We here recall that

$$
\operatorname{supp} c_{h}^{+} \subset\left\{(y, \xi) \in \mathbb{R}^{2 d} ; a_{h}^{+}\left(y, \partial_{\xi} S_{h}^{+}(y, \xi)\right) \neq 0\right\}
$$

(see, [24, Lemma 3.2] and its proof). In particular, $c_{h}^{+}(y, \xi)$ vanishes in the region $\{y ;|y| \geq 1 / h\}$. We now set $L=4 \sqrt{\sup I_{2}}+2$, where $I_{2}$ is given in Theorem 4.3. Since $|x| \geq L /(2 h),|y|<1 / h$ and $|\xi|^{2} \in I_{2}$ on the support of the amplitude $f_{h}(x) b_{h}^{+}(x, \xi) \overline{c_{h}^{+}(y, \xi)}$, we obtain

$$
\left|\partial_{\xi} \Phi_{h}^{+}(t, x, y, \xi)\right|>c\left(1+|x|+|y|+|\xi|+t+h^{-1}\right), \quad 0 \leq t \leq h^{-1},
$$

for some universal constant $c>0$. The assertion now follows from an integration by parts and the $L^{2}$-boundedness of $h$-FIO's. 
Proof of Lemma 4.4. The Duhamel formula yields

$$
\begin{aligned}
\left(e^{-i t H^{h} / h}-e^{-i t \widetilde{H}^{h} / h}\right)= & -\frac{i}{h} \int_{0}^{t} e^{-i(t-s) H^{h} / h} W_{0}^{h} e^{-i s \widetilde{H}^{h} / h} d s \\
= & -\frac{i}{h} \int_{0}^{t} e^{-i(t-s) H^{h} / h} e^{-i s \widetilde{H}^{h} / h} W_{0}^{h} d s \\
& +\frac{1}{h^{2}} \int_{0}^{t} e^{-i(t-s) H^{h} / h} \int_{0}^{s} e^{-i(s-\tau) \widetilde{H}^{h} / h}\left[\widetilde{H}^{h}, W_{0}^{h}\right] e^{-i \tau \widetilde{H}^{h} / h} d \tau d s,
\end{aligned}
$$

where $W_{0}^{h}:=H^{h}-\widetilde{H}^{h}$ consists of the following two parts:

$$
\begin{aligned}
& \frac{i h^{2}}{2} \sum_{j, k}\left(\partial_{j} g^{j k}(1-\psi(h x / L)) A_{k}+(1-\psi(h x / L)) A_{j} g^{j k} \partial_{k}\right), \\
& \frac{h^{2}}{2} \sum_{j, k}(1-\psi(h x / L))^{2} g^{j k} A_{j} A_{k}+h^{2}(1-\psi(h x / L)) V .
\end{aligned}
$$

In particular, $W_{0}^{h}$ is a first order differential operator of the form

$$
h^{2} \sum_{|\alpha|=1} f_{\alpha}^{h}(x) \partial_{x}^{\alpha}+h^{2} f_{0}^{h}(x)
$$

where $f_{\alpha}^{h}, f_{0}^{h}$ are supported in $\{|x| \geq L /(2 h)\}$ and satisfy

$$
\left|\partial_{x}^{\beta} f_{\alpha}^{h}(x)\right| \leq C_{\alpha \beta}\langle x\rangle^{1-\mu-|\beta|},\left|\partial_{x}^{\beta} f_{0}^{h}(x)\right| \leq C_{\alpha \beta}\langle x\rangle^{2-\mu-|\beta|} .
$$

Since $\{|x| \geq L /(2 h)\} \cap \pi_{x}\left(\operatorname{supp} a_{h}^{+}\right)=\emptyset$ if $L>1$, we have

$$
\left\|W_{0}^{h} \mathrm{Op}_{h}\left(a_{h}^{+}\right)^{*}\langle D\rangle^{s}\right\|_{L^{2} \rightarrow L^{2}} \leq C_{M, s} h^{M-s}, \quad M \geq 0, s \in \mathbb{R} .
$$

Therefore, the first term of the right hand side of the above Duhamel formula satisfies the desired estimates since $e^{-i t H^{h} / h}$ and $e^{-i t \widehat{H}^{h} / h}$ are unitary on $L^{2}$.

We next study the second term. Again by the Duhamel formula, we have

$$
\left[\widetilde{H}^{h}, W_{0}^{h}\right] e^{-i \tau \widetilde{H}^{h} / h}=e^{-i \tau \widetilde{H}^{h} / h}\left[\widetilde{H}^{h}, W_{0}^{h}\right]+\frac{i}{h} \int_{0}^{\tau} e^{-i(\tau-u) \widetilde{H}^{h} / h}\left[\widetilde{H}^{h},\left[\widetilde{H}^{h}, W_{0}^{h}\right]\right] e^{-i u \widetilde{H}^{h} / h} d u
$$

Since the coefficients of the commutator $\left[\widetilde{H}^{h}, W_{0}^{h}\right]$ are supported in $\{|x| \geq L /(2 h)\}$, the support property of $a_{h}^{+}$again implies that $\left[\widetilde{H}^{h}, W_{0}^{h}\right] \mathrm{Op}_{h}\left(a_{h}^{+}\right)^{*}\langle D\rangle^{s}=O_{L^{2} \rightarrow L^{2}}\left(h^{M-s}\right)$ for any $M \geq 0$ and $s \in \mathbb{R}$. Furthermore, by virtue of (4.1), (4.8) and the symbolic calculus, the coefficients of $\left[\widetilde{H}^{h},\left[\widetilde{H}^{h}, W_{0}^{h}\right]\right]$ are uniformly bounded in $x$ and supported in $\{|x| \geq L /(2 h)\}$. We now apply Lemma 4.5 to $\left[\widetilde{H}^{h},\left[\widetilde{H}^{h}, W_{0}^{h}\right]\right] e^{-i u \widetilde{H}^{h} / h} \mathrm{Op}_{h}\left(a_{h}^{+}\right)^{*}$ and obtain the assertion.

We now come into the proof of Proposition 3.1.

Proof of Proposition 3.1. Rescaling $t \rightarrow t h$, it suffices to show

$$
\left\|\mathrm{Op}_{h}\left(a_{h}^{+}\right) e^{-i t H^{h} / h} \mathrm{Op}_{h}\left(a_{h}^{+}\right)^{*}\right\|_{L^{1} \rightarrow L^{\infty}} \leq C_{\varepsilon}|t h|^{-d / 2}, \quad 0<|t| \leq h^{-1},
$$


where $H^{h}=h^{2} H$. Let $A_{h}(x, y)$ be the distribution kernel of $\mathrm{Op}_{h}\left(a_{h}^{+}\right)$:

$$
A_{h}(x, y)=(2 \pi h)^{-d} \int e^{i(x-y) \cdot \xi / h} a_{h}^{+}(x, \xi) d \xi .
$$

Since $a_{h}^{+} \in S(1, g)$ is compactly supported in $I$ with respect to $\xi$, we easily see that

$$
\sup _{x} \int\left|A_{h}(x, y)\right| d y+\sup _{y} \int\left|A_{h}(x, y)\right| d x \leq C, \quad h \in(0,1] .
$$

Moreover, since $\langle\xi\rangle^{s} a_{h}^{+}\langle\xi\rangle^{\gamma} \in S(1, g)$ for any $s, \gamma$, we have

$$
\left\|\langle D\rangle^{s} \mathrm{Op}_{h}\left(a_{h}^{+}\right)\langle D\rangle^{\gamma}\right\|_{L^{2} \rightarrow L^{2}} \leq C_{s} h^{-s-\gamma} .
$$

Combining these two estimates with Theorem 4.3 and Lemma 4.4, we can write

$$
\mathrm{Op}_{h}\left(a_{h}^{+}\right) e^{-i t H^{h} / h} \mathrm{Op}_{h}\left(a_{h}^{+}\right)^{*}=K_{1}(t, h, N)+K_{2}(t, h, N),
$$

where

$$
\begin{aligned}
& K_{1}(t, h, N)=\operatorname{Op}_{h}\left(a_{h}^{+}\right) J_{h}\left(S_{h}^{+}, b_{h}^{+}\right) e^{-i t H_{0}^{h} / h} J_{h}\left(S_{h}^{+}, c_{h}^{+}\right)^{*} \\
& K_{2}(t, h, N)=\operatorname{Op}_{h}\left(a_{h}^{+}\right) Q_{\mathrm{IK}}^{+}(t, h, N)+\operatorname{Op}_{h}\left(a_{h}^{+}\right)\left(e^{-i t H^{h} / h}-e^{-i t \widetilde{H}^{h} / h}\right) \operatorname{Op}_{h}\left(a_{h}^{+}\right)^{*} .
\end{aligned}
$$

By (4.7), the distribution kernel of $K_{1}(t, h, N)$, which we denote by $K_{1}(t, x, y)$, satisfies

$$
\left|K_{1}(t, x, y)\right| \leq \int\left|A_{h}(x, z)\right|\left|K_{S_{h}^{+}}(t, z, y)\right| d z \leq C_{N}|t h|^{-d / 2}, \quad 0<t \leq h^{-1},
$$

uniformly in $h \in\left(0, h_{0}\right]$. On the other hand, (4.6), Lemma 4.4 and (4.9) imply

$$
\left\|\langle D\rangle^{s} K_{2}(t, h, N)\langle D\rangle^{s}\right\|_{L^{2} \rightarrow L^{2}} \leq C_{N, s} h^{N-2 s-1} .
$$

If we choose $N \geq d+2$ and $s>d / 2$, then it follows from the Sobolev embedding that the distribution kernel of $K_{2}(t, h, N)$ is uniformly bounded in $\mathbb{R}^{2 d}$ with respect to $h \in\left(0, h_{0}\right]$ and $0<$ $t \leq h^{-1}$. Therefore, $\mathrm{Op}_{h}\left(a_{h}^{+}\right) e^{-i t H^{h} / h} \mathrm{Op}_{h}\left(a_{h}^{+}\right)^{*}$ has the distribution kernel $K(t, x, y)$ satisfying dispersive estimates for $0<t \leq h^{-1}$ :

$$
|K(t, x, y)| \leq C_{N}|t h|^{-d / 2}, \quad x, y \in \mathbb{R}^{d} .
$$

Finally, using the following relation,

$$
\mathrm{Op}_{h}\left(a_{h}^{+}\right) e^{-i t H^{h} / h} \mathrm{Op}_{h}\left(a_{h}^{+}\right)^{*}=\left(\mathrm{Op}_{h}\left(a_{h}^{+}\right) e^{i t H^{h} / h} \mathrm{Op}_{h}\left(a_{h}^{+}\right)^{*}\right)^{*}
$$

we learn $K(t, x, y)=\overline{K(-t, y, x)}$ and (4.10) also holds for $0<-t \leq h^{-1}$. For the incoming case, the proof is analogous and we omit it. 


\section{Fourier integral operators with the time dependent phase}

Throughout this section we assume Assumption 1.1 with $\mu \geq 0$. Consider a symbol $\chi_{\varepsilon} \in S(1, g)$ supported in a region

$$
\Omega(\varepsilon):=\left\{(x, \xi) \in \mathbb{R}^{2 d} ;\langle x\rangle>\varepsilon|\xi| / 2\right\},
$$

where $\varepsilon>0$ is an arbitrarily small fixed constant. In this section we prove the following dispersive estimate:

$$
\left\|\mathrm{Op}\left(\chi_{\varepsilon}\right) e^{-i t H} \mathrm{Op}\left(\chi_{\varepsilon}\right)^{*}\right\|_{L^{1} \rightarrow L^{\infty}} \leq C_{\varepsilon}|t|^{-d / 2}, \quad 0<|t| \leq t_{\varepsilon},
$$

where $t_{\varepsilon}>0$ is a small constant depending on $\varepsilon$. This estimate, combined with the $L^{2}$ boundedness of $\operatorname{Op}\left(\chi_{\varepsilon}\right)$ and $e^{-i t H}$, implies Strichartz estimates for $\operatorname{Op}\left(\chi_{\varepsilon}\right) e^{-i t H}$.

Let us give a short summary of the steps of proof. Choose $\chi_{\varepsilon}^{*} \in S(1, g)$ so that $\operatorname{supp} \chi_{\varepsilon}^{*}=$ $\operatorname{supp} \chi_{\varepsilon}$ and $\operatorname{Op}\left(\chi_{\varepsilon}\right)^{*}=\operatorname{Op}\left(\chi_{\varepsilon}^{*}\right)+\mathrm{Op}\left(r_{N}\right)$ with some $r_{N} \in S\left(\langle x\rangle^{-N}\langle\xi\rangle^{-N}, g\right)$ for sufficiently large $N>d / 2$. We first construct an approximation for $e^{-i t H} \mathrm{Op}\left(\chi_{\varepsilon}^{*}\right)$ in terms of the FIO with a time dependent phase:

$$
J\left(\Psi, b^{N}\right) f(x)=\frac{1}{(2 \pi)^{d}} \int e^{i(\Psi(t, x, \xi)-y \cdot \xi)} b(t, x, \xi) f(y) d y d \xi
$$

where $\Psi$ is a generating function of the Hamilton flow associated to $p(x, \xi)$ and $\left(\partial_{\xi} \Psi, \xi\right) \mapsto$ $\left(x, \partial_{x} \Psi\right)$ is the corresponding canonical map, and the amplitude $b=b_{0}+b_{2}+\cdots+b_{N-1}$ solves the corresponding transport equations. Although such parametrix constructions are well known as WKB approximations (at least if $\chi_{\varepsilon}^{*}$ is compactly supported in $\xi$ and the time scale depends on the size of frequency), we give the detail of proof since, in the present case, $\operatorname{supp} \chi_{\varepsilon}^{*}$ is not compact with respect to $\xi$ and $t_{\varepsilon}$ is independent of the size of frequency. The crucial point is that $p(x, \xi)$ is of quadratic type on $\Omega(\varepsilon)$ :

$$
\left|\partial_{x}^{\alpha} \partial_{\xi}^{\beta} p(x, \xi)\right| \leq C_{\alpha \beta}, \quad(x, \xi) \in \Omega(\varepsilon),|\alpha+\beta| \geq 2,
$$

which allows us to follow a classical argument (due to, e.g., [20]) and construct the approximation for $|t|<t_{\varepsilon}$ if $t_{\varepsilon}>0$ is small enough. The composition $\operatorname{Op}\left(\chi_{\varepsilon}\right) J(\Psi, b)$ is also a FIO with the same phase, and a standard stationary phase method can be used to prove dispersive estimates for $0<|t|<t_{\varepsilon}$. It remains to obtain the $L^{1} \rightarrow L^{\infty}$ bounds of the remainders $\operatorname{Op}\left(\chi_{\varepsilon}\right) e^{-i t H} \operatorname{Op}\left(r_{N}\right)$ and $\operatorname{Op}\left(\chi_{\varepsilon}\right) e^{-i t H}\left(\operatorname{Op}\left(\chi_{\varepsilon}^{*}\right)-J\left(\Psi, b^{N}\right)\right)$. If $e^{-i t H}$ maps from the Sobolev space $H^{d / 2}\left(\mathbb{R}^{d}\right)$ to itself, then $L^{1} \rightarrow L^{\infty}$ bounds are direct consequences of the Sobolev embedding and $L^{2}$-boundedness of PDO. However, our Hamiltonian $H$ is not bounded below (on $\{|x| \gtrsim|\xi|\}$ ) and such a property does not hold in general. To overcome this difficulty, we use an Egorov type lemma as follows. By the Sobolev embedding and the Littlewood-Paley decomposition, the proof is reduced to that of the following estimates:

$$
\sum_{j \geq 0}\left\|2^{j \gamma} S_{j}(D) \operatorname{Op}\left(\chi_{\varepsilon}\right) e^{-i t H} \mathrm{Op}\left(r_{N}\right)\langle D\rangle^{\gamma} f\right\|_{L^{2}}^{2} \leq C\|f\|_{L^{2}}^{2},
$$

where $\gamma>d / 2$ and $S_{j}$ is a dyadic partition of unity. Then, we will prove that there exists $\eta_{j}(t, \cdot, \cdot) \in S(1, g)$ such that

$$
2^{j} \leq C(1+|x|+|\xi|) \quad \text { on } \operatorname{supp} \eta_{j}(t)
$$


and that

$$
S_{j}(D) \operatorname{Op}\left(\chi_{\varepsilon}\right) e^{-i t H}=e^{-i t H} \mathrm{Op}\left(\eta_{j}(t)\right)+O_{L^{2} \rightarrow L^{2}}\left(2^{-j N}\right), \quad|t|<t_{\varepsilon} \ll 1 .
$$

Choosing $\delta>0$ with $\gamma+\delta \leq N / 2$, we learn that $2^{j(\gamma+\delta)} \eta_{j}(t) r_{N}\langle\xi\rangle^{\gamma} \in S(1, g)$ and hence (5.1). $\mathrm{Op}\left(\chi_{\varepsilon}\right) e^{-i t H}\left(\mathrm{Op}\left(\chi_{\varepsilon}^{*}\right)-J(\Psi, b)\right)$ can be controlled similarly.

\subsection{Short-time behavior of the Hamilton flow}

This subsection discusses the classical mechanics generated by $p(x, \xi)$. We denote the solution to the following Hamilton equations by $(X(t), \Xi(t))=(X(t, x, \xi), \Xi(t, x, \xi))$ :

$$
\left\{\begin{aligned}
& \dot{X}_{j}=\frac{\partial p}{\partial \xi_{j}}(X, \Xi)=\sum_{k} g^{j k}(X)\left(\Xi_{k}-A_{k}(X)\right), \\
& \dot{\Xi}_{j}=-\frac{\partial p}{\partial x_{j}}(X, \Xi)=-\frac{1}{2} \sum_{k, l} \frac{\partial g^{k l}}{\partial x_{j}}(X)\left(\Xi_{k}-A_{k}(X)\right)\left(\Xi_{l}-A_{l}(X)\right) \\
&+\sum_{k, l} g^{k l}(X) \frac{\partial A_{k}}{\partial x_{j}}(X)\left(\Xi_{l}-A_{l}(X)\right)-\frac{\partial V}{\partial x_{j}}(X)
\end{aligned}\right.
$$

with the initial condition $(X(0), \Xi(0))=(x, \xi)$, where $\dot{f}=\partial_{t} f$. We first observe that the flow conserves the energy:

$$
p(x, \xi)=p(X(t), \Xi(t)),
$$

which, combined with the uniform ellipticity of $g^{j k}$, implies

$$
\begin{aligned}
|\Xi(t)-A(X(t))|^{2} & \lesssim p(X(t), \Xi(t))-V(X(t)) \\
& =p(x, \xi)-V(X(t)) \\
& \lesssim|\xi-A(x)|^{2}+|V(x)|+|V(X(t))|,
\end{aligned}
$$

and hence $|\Xi(t)| \lesssim|\xi|+\langle x\rangle+\langle X(t)\rangle$. By the Hamilton equation, we then have

$$
|\dot{X}(t)|+|\dot{\Xi}(t)| \leq C(1+|\xi|+|x|+|X(t)|+|\Xi(t)|) .
$$

Applying Gronwall's inequality to this estimate, we obtain an a priori bound:

$$
|X(t)-x|+|\Xi(t)-\xi| \leq C_{T}|t|(1+|x|+|\xi|), \quad|t| \leq T, x, \xi \in \mathbb{R}^{d} .
$$

Using this estimate, we obtain more precise behavior of the flow with initial conditions in $\Omega(\varepsilon)$.

Lemma 5.1. Let $\varepsilon>0$. Then, for sufficiently small $t_{\varepsilon}>0$ and all $\alpha, \beta \in \mathbb{Z}_{+}^{d}$,

$$
\left|\partial_{x}^{\alpha} \partial_{\xi}^{\beta}(X(t, x, \xi)-x)\right|+\mid \partial_{x}^{\alpha} \partial_{\xi}^{\beta}\left(\Xi(t, x, \xi)-\xi\left|\leq C_{\alpha \beta \varepsilon}\right| t \mid\langle x\rangle^{1-|\alpha+\beta|},\right.
$$

uniformly with respect to $(t, x, \xi) \in\left(-t_{\varepsilon}, t_{\varepsilon}\right) \times \Omega(\varepsilon)$.

Proof. We only consider the case with $t \geq 0$, the proof for the opposite case is similar. Let $(x, \xi) \in \Omega(\varepsilon)$. At first we remark that for sufficiently small $t_{\varepsilon}>0$,

$$
\langle x\rangle / 2 \leq|X(t, x, \xi)| \leq 2\langle x\rangle, \quad|t| \leq t_{\varepsilon} .
$$


For $|\alpha+\beta|=0$, the assertion is obvious. We let $|\alpha+\beta|=1$ and differentiate the Hamilton equations with respect to $\partial_{x}^{\alpha} \partial_{\xi}^{\beta}$ :

$$
\frac{d}{d t}\left(\begin{array}{c}
\partial_{x}^{\alpha} \partial_{\xi}^{\beta} X \\
\partial_{x}^{\alpha} \partial_{\xi}^{\beta} \Xi
\end{array}\right)=\left(\begin{array}{cc}
\partial_{x} \partial_{\xi} p(X, \Xi) & \partial_{\xi}^{2} p(X, \Xi) \\
-\partial_{x}^{2} p(X, \Xi) & -\partial_{\xi} \partial_{x} p(X, \Xi)
\end{array}\right)\left(\begin{array}{c}
\partial_{x}^{\alpha} \partial_{\xi}^{\beta} X \\
\partial_{x}^{\alpha} \partial_{\xi}^{\beta} \Xi
\end{array}\right) .
$$

Using (5.2), we learn that $p(X(t), \Xi(t))$ is of quadratic type in $\Omega(\varepsilon)$ :

$$
\left|\left(\partial_{x}^{\alpha} \partial_{\xi}^{\beta} p\right)(X(t), \Xi(t))\right| \leq C_{\alpha \beta \varepsilon}\langle x\rangle^{2-|\alpha+\beta|}, \quad(t, x, \xi) \in\left(-t_{\varepsilon}, t_{\varepsilon}\right) \times \Omega(\varepsilon) .
$$

All entries of the above matrix hence are uniformly bounded in $(t, x, \xi) \in\left(-t_{\varepsilon}, t_{\varepsilon}\right) \times \Omega(\varepsilon)$. Taking $t_{\varepsilon}>0$ smaller if necessary, integrating (5.3) with respect to $t$ and applying Gronwall's inequality, we have the assertion with $|\alpha+\beta|=1$. For $|\alpha+\beta| \geq 2$, we prove the estimate for $\partial_{\xi_{1}}^{2} X(t)$ and $\partial_{\xi_{1}}^{2} \Xi(t)$ only, where $\xi=\left(\xi_{1}, \xi_{2}, \ldots, \xi_{d}\right)$. Proofs for other cases are similar, and proofs for higher derivatives follow from an induction on $|\alpha+\beta|$. By the Hamilton equation, we learn

$$
\frac{d}{d t} \partial_{\xi_{1}}^{2} X(t)=\partial_{x} \partial_{\xi} p(X(t), \Xi(t)) \partial_{\xi_{1}}^{2} X(t)+\partial_{\xi}^{2} p(X(t), \Xi(t)) \partial_{\xi_{1}}^{2} \Xi(t)+Q(X(t), \Xi(t)),
$$

where $Q(X(t), \Xi(t))$ satisfies

$$
\begin{aligned}
|Q(X(t), \Xi(t))| & \leq C_{\varepsilon} \sum_{|\alpha+\beta|=3,|\beta| \geq 1}\left|\left(\partial_{x}^{\alpha} \partial_{\xi}^{\beta} p\right)(X(t), \Xi(t))\right|\left|\partial_{\xi_{1}} X(t)\right|^{|\alpha|}\left|\partial_{\xi_{1}} \Xi(t)\right|^{|\beta|} \\
& \leq C_{\varepsilon}\langle x\rangle^{-1} .
\end{aligned}
$$

We similarly obtain

$$
\frac{d}{d t} \partial_{\xi_{1}}^{2} \Xi(t)=-\partial_{x}^{2} p(X(t), \Xi(t)) \partial_{\xi_{1}}^{2} X(t)-\partial_{\xi} \partial_{x} p(X(t), \Xi(t)) \partial_{\xi_{1}}^{2} \Xi(t)+O\left(\langle x\rangle^{-1}\right) .
$$

Applying Gronwall's inequality, we have the desired estimates.

Lemma 5.2. (1) Let $t_{\varepsilon}>0$ be small enough. Then, for any $|t|<t_{\varepsilon}$, the map

$$
g(t):(x, \xi) \mapsto(X(t, x, \xi), \xi)
$$

is a diffeomorphism from $\Omega(\varepsilon / 2)$ onto its range, and satisfies

$$
\Omega(\varepsilon) \subset g(t, \Omega(\varepsilon / 2)) \text { for all }|t|<t_{\varepsilon} .
$$

(2) Let $\Omega(\varepsilon) \ni(x, \xi) \mapsto(Y(t, x, \xi), \xi) \in \Omega(\varepsilon / 2)$ be the inverse map of $g(t)$. Then, $Y(t, x, \xi)$ and $\Xi(t, Y(t, x, \xi), \xi)$ satisfy the same estimates as that for $X(t, x, \xi)$ and $\Xi(t, x, \xi)$ of Lemma 5.1 , respectively:

$$
\left|\partial_{x}^{\alpha} \partial_{\xi}^{\beta}(Y(t, x, \xi)-x)\right|+\mid \partial_{x}^{\alpha} \partial_{\xi}^{\beta}\left(\Xi(t, Y(t, x, \xi), \xi)-\xi\left|\leq C_{\alpha \beta \varepsilon}\right| t \mid\langle x\rangle^{1-|\alpha+\beta|},\right.
$$

uniformly with respect to $(t, x, \xi) \in\left(-t_{\varepsilon}, t_{\varepsilon}\right) \times \Omega(\varepsilon)$. 
Proof. Choosing a cut-off function $\rho \in S(1, g)$ such that $0 \leq \rho \leq 1$, supp $\rho \subset \Omega(\varepsilon / 3)$ and $\rho \equiv 1$ on $\Omega(\varepsilon / 2)$, we modify $g(t)$ as follows:

$$
g_{\rho}(t, x, \xi)=\left(X_{\rho}(t, x, \xi), \xi\right), X_{\rho}(t, x, \xi)=(1-\rho(x, \xi)) x+\rho(x, \xi) X(t, x, \xi) .
$$

It is easy to see that, for $(t, x, \xi) \in\left(-t_{\varepsilon}, t_{\varepsilon}\right) \times \Omega(\varepsilon / 2), g_{\rho}(t, x, \xi)$ is smooth and Lemma 5.1 implies

$$
\begin{aligned}
\left|\partial_{x}^{\alpha} \partial_{\xi}^{\beta} g_{\rho}(t, x, \xi)\right| & \leq C_{\alpha \beta \varepsilon}, \quad|\alpha+\beta| \geq 1, \\
\left|J\left(g_{\rho}\right)(t, x, \xi)-\mathrm{Id}\right| & \leq C_{\varepsilon} t_{\varepsilon}
\end{aligned}
$$

where $J\left(g_{\rho}\right)$ is the Jacobi matrix with respect to $(x, \xi)$ and the constant $C_{\varepsilon}>0$ is independent of $t, x$ and $\xi$. Choosing $t_{\varepsilon}>0$ so small that $C_{\varepsilon} t_{\varepsilon}<1 / 2$, and applying the Hadamard global inverse mapping theorem, we see that, for any fixed $|t|<t_{\varepsilon}, g_{\rho}(t)$ is a diffeomorphism from $\mathbb{R}^{2 d}$ onto itself. By definition, $g(t)$ is diffeomorphic from $\Omega(\varepsilon / 2)$ onto its range. Since $g_{\rho}(t)$ is bijective, it remains to check that

$$
\Omega(\varepsilon)^{c} \supset g_{\rho}\left(t, \Omega(\varepsilon / 2)^{c}\right), \quad|t|<t_{\varepsilon} .
$$

Suppose that $(x, \xi) \in \Omega(\varepsilon / 2)^{c}$. If $(x, \xi) \in \Omega(\varepsilon / 3)^{c}$, then the assertion is obvious since $g_{\rho}(t) \equiv \mathrm{Id}$ outside $\Omega(\varepsilon / 3)$. If $(x, \xi) \in \Omega(\varepsilon / 3) \backslash \Omega(\varepsilon / 2)$, then, by Lemma 5.1 and the support property of $\rho$, we have

$$
\left|X_{\rho}(t, x, \xi)\right| \leq|x|+\rho(x, \xi)|(X(t, x, \xi)-x)| \leq\left(\varepsilon / 2+C_{0} t_{\varepsilon}\right)\langle\xi\rangle
$$

for some $C_{0}>0$ independent of $x, \xi$ and $t_{\varepsilon}$. Choosing $t_{\varepsilon}<\varepsilon /\left(2 C_{0}\right)$, we obtain the assertion.

We next prove the estimates on $Y(t)$. Since $(Y(t, x, \xi), \xi) \in \Omega(\varepsilon / 2)$, we learn

$$
\begin{aligned}
|Y(t, x, \xi)-x| & =|X(0, Y(t, x, \xi), \xi)-X(t, Y(t, x, \xi), \xi)| \\
& \leq \sup _{(x, \xi) \in \Omega(\varepsilon / 2)}|X(t, x, \xi)-x| \\
& \leq C_{\varepsilon}|t|\langle x\rangle .
\end{aligned}
$$

For $\alpha, \beta \in \mathbb{Z}_{+}^{d}$ with $|\alpha+\beta|=1$, apply $\partial_{x}^{\alpha} \partial_{\xi}^{\beta}$ to the equality $x=X(t, Y(t, x, \xi), \xi)$. We then have the following equality

$$
A(t, Z(t, x, \xi)) \partial_{x}^{\alpha} \partial_{\xi}^{\beta}(Y(t, x, \xi)-x)=\left.\partial_{y}^{\alpha} \partial_{\eta}^{\beta}(y-X(t, y, \eta))\right|_{(y, \eta)=Z(t, x, \xi)},
$$

where $Z(t, x, \xi)=(Y(t, x, \xi), \xi)$ and $A(t, Z)=\left(\partial_{x} X\right)(t, Z)$ is a $d \times d$-matrix. By Lemma 5.1 and a similar argument as that in the proof of Lemma 5.2 (1), we learn that $A(t, Z(t, x, \xi))$ is invertible if $t_{\varepsilon}>0$ is small enough, and that $A(t, Z(t, x, \xi))$ and $A(t, Z(t, x, \xi))^{-1}$ are bounded uniformly in $(t, x, \xi) \in\left(-t_{\varepsilon}, t_{\varepsilon}\right) \times \Omega(\varepsilon / 2)$. Therefore,

$$
\begin{aligned}
\left|\partial_{x}^{\alpha} \partial_{\xi}^{\beta}(Y(t, x, \xi)-x)\right| & \leq C_{\alpha \beta} \sup _{(x, \xi) \in \Omega(\varepsilon / 2)}\left|\partial_{x}^{\alpha} \partial_{\xi}^{\beta}(x-X(t, x, \xi))\right| \\
& \leq C_{\alpha \beta}|t|\langle x\rangle^{1-|\alpha+\beta|}
\end{aligned}
$$

Proofs for higher derivatives are obtained by an induction with respect to $|\alpha+\beta|$ and proofs for $\Xi(t, Y(t, x, \xi), \xi)$ are similar. 


\subsection{The parametrix for $\mathrm{Op}\left(\chi_{\varepsilon}\right) e^{-i t H} \mathrm{Op}\left(\chi_{\varepsilon}\right)^{*}$}

Before starting the construction of parametrix, we prepare two lemmas. The following is an Egorov type theorem which will be used to control the remainder term. We write $\exp t H_{p}(x, \xi)=$ $(X(t, x, \xi), \Xi(t, x, \xi))$.

Lemma 5.3. For $h \in(0,1]$, consider a h-dependent symbol $\eta_{h} \in S(1, g)$ such that $\operatorname{supp} \eta_{h} \subset$ $\Omega(\varepsilon) \cap\{1 /(2 h)<|\xi|<2 / h\}$. Then, for sufficiently small $t_{\varepsilon}>0$, independent of $h$, and any integer $N \geq 0$, there exists a bounded family of symbols $\left\{\eta_{h}^{N}(t, \cdot, \cdot) ;|t|<t_{\varepsilon}, 0<h \leq 1\right\} \subset S(1, g)$ such that

$$
\operatorname{supp} \eta_{h}^{N}(t, \cdot, \cdot) \subset \exp (-t) H_{p}\left(\operatorname{supp} \eta_{h}\right)
$$

and that

$$
\left\|e^{i t H} \mathrm{Op}\left(\eta_{h}\right) e^{-i t H}-\mathrm{Op}\left(\eta_{h}^{N}(t)\right)\right\|_{L^{2} \rightarrow L^{2}} \leq C_{N \varepsilon} h^{N}
$$

uniformly with respect to $0<h \leq 1$ and $|t|<t_{\varepsilon}$.

Proof. Let $\eta_{h}^{0}(t, x, \xi)=\eta_{h}\left(\exp t H_{p}(x, \xi)\right)=\eta_{h}(X(t, x, \xi), \Xi(t, x, \xi))$. It is easy to see that $\operatorname{supp} \eta_{h}^{0} \subset \exp (-t) H_{p}\left(\operatorname{supp} \eta_{h}\right)$. Moreover, Lemma 5.1 implies that $\left\{\eta_{h}^{0} ;|t|<t_{\varepsilon}, 0<h \leq 1\right\}$ is a bounded subset of $S(1, g)$. By a direct computation, $\eta_{h}^{0}$ solves

$$
\partial_{t} \eta_{h}^{0}=\left\{p, \eta_{h}^{0}\right\} ;\left.\quad \eta_{h}^{0}\right|_{t=0}=\eta_{h},
$$

where $\{\cdot, \cdot\}$ is the Poisson bracket. Then, by a standard pseudodifferential calculus, there exists a bounded set $\left\{r_{h}^{0}(t, \cdot, \cdot) ; 0 \leq t<t_{\varepsilon}, 0<h \leq 1\right\} \subset S(1, g)$ with $\operatorname{supp} r_{h}^{0} \subset \exp (-t) H_{p}\left(\operatorname{supp} \eta_{h}\right)$ such that

$$
\frac{d}{d t} \mathrm{Op}\left(\eta_{h}^{0}\right)=i\left[H, \mathrm{Op}\left(\eta_{h}^{0}\right)\right]+h \mathrm{Op}\left(r_{h}^{0}\right)
$$

We next set

$$
\eta_{h}^{1}(t, x, \xi)=\int_{0}^{t} r_{h}^{0}(s, X(t-s, x, \xi), \Xi(t-s, x, \xi)) d s .
$$

Again, we learn that $\left\{\eta_{h}^{1}(t, \cdot, \cdot) ;|t|<t_{\varepsilon}, 0<h \leq 1\right\} \subset S(1, g)$ is also bounded and that $\operatorname{supp} \eta_{h}^{1} \subset$ $\exp (-t) H_{p}\left(\operatorname{supp} \eta_{h}\right)$ for all $|t|<t_{\varepsilon}$ and $0<h \leq 1$. Moreover, $\eta_{h}^{1}$ solves

$$
\partial_{t} \eta_{h}^{1}=\left\{p, \eta_{h}^{1}\right\}+r_{h}^{0} ;\left.\quad \eta_{h}^{1}\right|_{t=0}=0,
$$

which implies

$$
\frac{d}{d t} \mathrm{Op}\left(\eta_{h}^{0}+h \eta_{h}^{1}\right)=i\left[H, \mathrm{Op}\left(\eta_{h}^{0}+h \eta_{h}^{1}\right)\right]+h^{2} \mathrm{Op}\left(r_{h}^{1}\right) .
$$

with some $\left\{r_{h}^{1} ; 0 \leq t<t_{\varepsilon}, 0<h \leq 1\right\} \subset S(1, g)$ and $\operatorname{supp} r_{h}^{1} \subset \exp (-t) H_{p}\left(\operatorname{supp} \eta_{h}\right)$. Iterating this procedure and putting $\eta_{h}^{N}=\sum_{j=0}^{N-1} h^{j} \eta_{h}^{j}$, we obtain the assertion.

Using this lemma, we have the following.

Lemma 5.4. Let $\varepsilon>0$. Then, for any symbol $\chi_{\varepsilon} \in S(1, g)$ with $\operatorname{supp} \chi_{\varepsilon} \subset \Omega(\varepsilon)$ and any integer $N \geq 1$, there exists $\chi_{\varepsilon}^{*} \in S(1, g)$ with $\operatorname{supp} \chi_{\varepsilon}^{*} \subset \Omega(\varepsilon)$ such that for any $\gamma<N / 2$,

$$
\sup _{|t|<t_{\varepsilon}} \| \operatorname{Op}\left(\chi_{\varepsilon}\right) e^{-i t H} \mathrm{Op}\left(\chi_{\varepsilon}\right)^{*}-\left.\mathrm{Op}\left(\chi_{\varepsilon}\right) e^{-i t H} \mathrm{Op}\left(\chi_{\varepsilon}^{*}\right)\right|_{H^{-\gamma}\left(\mathbb{R}^{d}\right) \rightarrow H^{\gamma}\left(\mathbb{R}^{d}\right)} \leq C_{N \gamma \varepsilon}
$$


Proof. By the expansion formula (2.4), there exists $\chi_{\varepsilon}^{*} \in S(1, g)$ with $\operatorname{supp} \chi_{\varepsilon}^{*} \subset \Omega(\varepsilon)$ such that

$$
\mathrm{Op}\left(\chi_{\varepsilon}\right)^{*}=\mathrm{Op}\left(\chi_{\varepsilon}^{*}\right)+\mathrm{Op}\left(r_{0}(N)\right)
$$

with some $r_{0}(N) \in S\left(\langle x\rangle^{-N}\langle\xi\rangle^{-N}, g\right)$. For $\delta>0$ with $2 \gamma+\delta \leq N$, we split

$$
\begin{aligned}
& \langle D\rangle^{\gamma} \mathrm{Op}\left(\chi_{\varepsilon}\right) e^{-i t H} \operatorname{Op}\left(r_{0}(N)\right)\langle D\rangle^{\gamma} \\
& =\langle D\rangle^{\gamma} \operatorname{Op}\left(\chi_{\varepsilon}\right) e^{-i t H}\langle D\rangle^{-\gamma-\delta}\langle x\rangle^{-\gamma-\delta} \cdot\langle x\rangle^{\gamma+\delta}\langle D\rangle^{\gamma+\delta} \mathrm{Op}\left(r_{0}(N)\right)\langle D\rangle^{\gamma}
\end{aligned}
$$

Since $\langle x\rangle^{\gamma+\delta}\langle\xi\rangle^{\gamma+\delta} r_{0}(N)\langle\xi\rangle^{\gamma} \in S(1, g),\langle x\rangle^{\gamma+\delta}\langle D\rangle^{\gamma+\delta} \mathrm{Op}\left(r_{0}(N)\right)\langle D\rangle^{\gamma}$ is bounded on $L^{2}$. In order to prove the $L^{2}$-boundedness of the first term of the right hand side, we use the standard Littlewood-Paley decomposition and Lemma 5.3 as follows. Consider a dyadic partition of unity with respect to the frequency:

$$
\sum_{j=0}^{\infty} S_{j}(D)=1,
$$

where $S_{j}(\xi)=S\left(2^{-j} \xi\right), j \geq 1$, with some $S \in C_{0}^{\infty}\left(\mathbb{R}^{d}\right)$ supported in $\{1 / 2<|\xi|<2\}$ and $S_{0} \in C_{0}^{\infty}\left(\mathbb{R}^{d}\right)$ supported in $\{|\xi|<1\}$. Then,

$$
\begin{aligned}
& \left\|\langle D\rangle^{\gamma} \operatorname{Op}\left(\chi_{\varepsilon}\right) e^{-i t H}\langle D\rangle^{-\gamma-\delta}\langle x\rangle^{-\gamma-\delta} f\right\|_{L^{2}} \\
& \leq C\left(\sum_{j=0}^{\infty}\left\|2^{j \gamma} S_{j}(D) \operatorname{Op}\left(\chi_{\varepsilon}\right) e^{-i t H}\langle D\rangle^{-\gamma-\delta}\langle x\rangle^{-\gamma-\delta} f\right\|_{L^{2}}^{2}\right)^{1 / 2} .
\end{aligned}
$$

By the expansion formula (2.3), there exists a sequence of symbols $\eta_{j} \in S(1, g)$ supported in $\Omega(\varepsilon) \cap\left\{2^{j-1}<|\xi|<2^{j+1}\right\}$ such that

$$
S_{j}(D) \operatorname{Op}\left(\chi_{\varepsilon}\right)=\operatorname{Op}\left(\eta_{j}\right)+Q_{1}(j, N), \quad\left\|Q_{1}(j, N)\right\|_{L^{2} \rightarrow L^{2}}=O\left(2^{-j N}\right) .
$$

We then learn by Lemma 5.3 with $h=2^{-j}$ that there exists $\left\{\eta_{j}^{N}(t) ;|t|<t_{\varepsilon}\right\} \subset S(1, g)$ such that

$$
\mathrm{Op}\left(\eta_{j}\right) e^{-i t H}=e^{-i t H} \mathrm{Op}\left(\eta_{j}^{N}(t)\right)+Q_{2}(t, j, N), \quad \sup _{|t|<t_{\varepsilon}}\left\|Q_{2}(t, j, N)\right\|_{L^{2} \rightarrow L^{2}}=O\left(2^{-j N}\right) .
$$

Since $N \geq \gamma+\delta$, the remainder satisfies

$$
\sup _{|t|<t_{\varepsilon}}\left\|2^{j \gamma}\left(Q_{1}(j, N) e^{-i t H}+Q_{2}(t, j, N)\right)\langle D\rangle^{-\gamma-\delta}\langle x\rangle^{-\gamma-\delta} f\right\|_{L^{2}}^{2} \leq C 2^{-2 j \delta}\|f\|_{L^{2}}^{2} .
$$

Suppose that $(x, \xi) \in \operatorname{supp} \eta_{j}^{N}(t)$. Since $\operatorname{supp} \eta_{j}^{N}(t) \subset \exp (-t) H_{p}\left(\operatorname{supp} \eta_{j}\right)$, we have

$$
|X(t, x, \xi)|>\varepsilon\langle\Xi(t, x, \xi)\rangle, 2^{j-1}<|\Xi(t, x, \xi)|<2^{j+1} .
$$

Using Lemma 5.1 with the initial data $(X(t, x, \xi), \Xi(t, x, \xi))$, we learn

$$
|x-X(t, x, \xi)|+|\xi-\Xi(t, x, \xi)| \leq C t_{\varepsilon}\langle X(t, x, \xi)\rangle, \quad|t|<t_{\varepsilon} .
$$

Combining these two estimates, we see that

$$
2^{j} \leq C(1+|x|+|\xi|), \quad(x, \xi) \in \operatorname{supp} \eta_{j}^{N}(t),|t|<t_{\varepsilon},
$$


where the constant $C>0$ is independent of $x, \xi$ and $t$, provided that $t_{\varepsilon}>0$ is small enough. Therefore, $2^{j(\gamma+\delta)} \eta_{j}^{N}(t)\langle\xi\rangle^{-\gamma-\delta}\langle x\rangle^{-\gamma-\delta} \in S(1, g)$ and the corresponding PDO is bounded on $L^{2}$. Finally, we obtain

$$
\begin{aligned}
& \sum_{j=0}^{\infty}\left\|2^{j \gamma} \mathrm{Op}\left(\eta_{j}\right) e^{-i t H}\langle D\rangle^{-\gamma-\delta}\langle x\rangle^{-\gamma-\delta} f\right\|_{L^{2}}^{2} \\
& \leq C \sum_{j=0}^{\infty}\left(\left\|2^{-j \delta} 2^{j(\gamma+\delta)} \mathrm{Op}\left(\eta_{j}^{N}(t)\right)\langle D\rangle^{-\gamma-\delta}\langle x\rangle^{-\gamma-\delta} f\right\|_{L^{2}}^{2}+2^{-2 j \delta}\|f\|_{L^{2}}^{2}\right) \\
& \leq C \sum_{j=0}^{\infty} 2^{-2 j \delta}\|f\|_{L^{2}}^{2} \\
& \leq C\|f\|_{L^{2}}^{2},
\end{aligned}
$$

which completes the proof.

We next consider a parametrix construction of $\mathrm{Op}\left(\chi_{\varepsilon}\right) e^{-i t H} \mathrm{Op}\left(\chi_{\varepsilon}^{*}\right)$. Let us first make the following ansatz:

$$
v(t, x)=\frac{1}{(2 \pi)^{d}} \int e^{i(\Psi(t, x, \xi)-y \cdot \xi)} b^{N}(t, x, \xi) f(y) d y d \xi,
$$

where $b^{N}=\sum_{j=0}^{N-1} b_{j}$. In order to approximately solve the Schrödinger equation

$$
i \partial_{t} v(t)=H v(t) ;\left.\quad v\right|_{t=0}=\operatorname{Op}\left(\chi_{\varepsilon}^{*}\right) \varphi
$$

the phase function $\Psi$ and the amplitude $b^{N}$ should satisfy the following Hamilton-Jacobi equation and transport equations, respectively:

$$
\begin{gathered}
\partial_{t} \Psi+p\left(x, \partial_{x} \Psi\right)=0 ;\left.\quad \Psi\right|_{t=0}=x \cdot \xi, \\
\left\{\begin{array}{l}
\partial_{t} b_{0}+X \cdot \partial_{x} b_{0}+y b_{0}=0 ;\left.\quad b_{0}\right|_{t=0}=\chi_{\varepsilon}, \\
\partial_{t} b_{j}+X \cdot \partial_{x} b_{j}+y b_{j}+i K b_{j-1}=0 ;\left.\quad b_{j}\right|_{t=0}=0, \quad 1 \leq j \leq N-1,
\end{array}\right.
\end{gathered}
$$

where $K$ is the kinetic energy part of $H$, a vector field $X$ and a function $y$ are defined by

$$
\begin{aligned}
x_{j}(t, x, \xi) & :=\left(\partial_{\xi_{j}} p\right)\left(x, \partial_{x} \Psi(t, x, \xi)\right), j=1, \ldots, d, \\
y(t, x, \xi) & :=\left[k\left(x, \partial_{x}\right) \Psi+p_{1}\left(x, \partial_{x} \Psi\right)\right](t, x, \xi) .
\end{aligned}
$$

Here $p, p_{1}$ are given by (1.6). We first construct the phase function $\Psi$.

Proposition 5.5. Let us fix $\varepsilon>0$ arbitrarily. Then, for sufficiently small $t_{\varepsilon}>0$, we can construct a smooth and real-valued function $\Psi \in C^{\infty}\left(\left(-t_{\varepsilon}, t_{\varepsilon}\right) \times \mathbb{R}^{2 d} ; \mathbb{R}\right)$ which solves the HamiltonJacobi equation (5.4) for $(x, \xi) \in \Omega(\varepsilon)$ and $|t| \leq t_{\varepsilon}$. Moreover, for all $\alpha, \beta \in \mathbb{Z}_{+}^{d}, x, \xi \in \mathbb{R}^{d}$ and $|t| \leq t_{\varepsilon}$

$$
\mid \partial_{x}^{\alpha} \partial_{\xi}^{\beta}\left(\Psi(t, x, \xi)-x \cdot \xi+\left.t p(x, \xi)\left|\leq C_{\alpha \beta \varepsilon}\right| t\right|^{2}\langle x\rangle^{2-|\alpha+\beta|},\right.
$$

where $C_{\alpha \beta \varepsilon}>0$ is independent of $x, \xi$ and $t$. 
Proof. We consider the case when $t \geq 0$, and the proof for $t \leq 0$ is similar. We first define the action integral $\widetilde{\Psi}(t, x, \xi)$ on $\left[0, t_{\varepsilon}\right) \times \Omega(\varepsilon / 2)$ by

$$
\widetilde{\Psi}(t, x, \xi):=x \cdot \xi+\int_{0}^{t} L(X(s, Y(t, x, \xi), \xi), \Xi(s, Y(t, x, \xi), \xi)) d s
$$

where $L(x, \xi)=\xi \cdot \partial_{\xi} p(x, \xi)-p(x, \xi)$ is the Lagrangian associated to $p(x, \xi)$, and $X, \Xi$ and $Y$ are given by Lemma $5.2(2)$ with $\varepsilon$ replaced by $\varepsilon / 2$. The smoothness of $\widetilde{\Psi}(t, x, \xi)$ follows from corresponding properties of $X(t), \Xi(t)$ and $Y(t)$. It is well known that $\widetilde{\Psi}(t, x, \xi)$ solves the Hamilton-Jacobi equation

$$
\partial_{t} \widetilde{\Psi}(t, x, \xi)+p\left(x, \partial_{x} \widetilde{\Psi}(t, x, \xi)\right)=0 ;\left.\quad \Psi\right|_{t=0}=x \cdot \xi,
$$

for $(x, \xi) \in \Omega(\varepsilon / 2)$, and satisfies

$$
\partial_{x} \widetilde{\Psi}(t, x, \xi)=\Xi(t, Y(t, x, \xi), \xi), \quad \partial_{\xi} \widetilde{\Psi}(t, x, \xi)=Y(t, x, \xi) .
$$

Lemma $5.2(2)$ shows that $p(Y(t, x, \xi), \xi)$ is of quadratic type:

$$
\left|\partial_{x}^{\alpha} \partial_{\xi}^{\beta} p(Y(t, x, \xi), \xi)\right| \leq C_{\alpha \beta \varepsilon}\langle x\rangle^{2-|\alpha+\beta|}, \quad(t, x, \xi) \in\left[0, t_{\varepsilon}\right) \times \Omega(\varepsilon / 2),
$$

which, combined with the energy conservation

$$
p\left(x, \partial_{x} \widetilde{\Psi}(t, x, \xi)\right)=p(Y(t, x, \xi), \xi),
$$

imply

$$
\left|\partial_{x}^{\alpha} \partial_{\xi}^{\beta}(\widetilde{\Psi}(t, x, \xi)-x \cdot \xi)\right| \leq C_{\alpha \beta \varepsilon}|t|\langle x\rangle^{2-|\alpha+\beta|}, \quad(t, x, \xi) \in\left[0, t_{\varepsilon}\right) \times \Omega(\varepsilon / 2) .
$$

We similarly obtain, for $(t, x, \xi) \in\left[0, t_{\varepsilon}\right) \times \Omega(\varepsilon / 2)$,

$$
\begin{aligned}
& \left|p\left(x, \partial_{x} \widetilde{\Psi}(t, x, \xi)\right)-p(x, \xi)\right| \\
& \left.\left.=\mid\left(\partial_{x} \widetilde{\Psi}(t, x, \xi)\right)-\xi\right) \cdot \int_{0}^{1}\left(\partial_{\xi} p\right)\left(x, \theta \partial_{x} \widetilde{\Psi}(t, x, \xi)\right)+(1-\theta) \xi\right) d \theta \mid \\
& \leq C_{\varepsilon}|t|\langle x\rangle^{2}
\end{aligned}
$$

and, more generally,

$$
\left|\partial_{x}^{\alpha} \partial_{\xi}^{\beta}\left(p\left(x, \partial_{x} \widetilde{\Psi}(t, x, \xi)\right)-p(x, \xi)\right)\right| \leq C_{\alpha \beta \varepsilon}|t|\langle x\rangle^{2-|\alpha+\beta|} .
$$

Therefore, integrating the Hamilton-Jacobi equation with respect to $t$, we have

$$
\left|\partial_{x}^{\alpha} \partial_{\xi}^{\beta}(\widetilde{\Psi}(t, x, \xi)-x \cdot \xi+t p(x, \xi))\right| \leq C_{\alpha \beta \varepsilon}|t|^{2}\langle x\rangle^{2-|\alpha+\beta|} .
$$

Finally, choosing a cut-off function $\rho \in S(1, g)$ so that $0 \leq \rho \leq 1, \rho \equiv 1$ on $\Omega(\varepsilon)$ and $\operatorname{supp} \rho \subset$ $\Omega(\varepsilon / 2)$, we define

$$
\Psi(t, x, \xi):=x \cdot \xi-t p(x, \xi)+\rho(x, \xi)(\widetilde{\Psi}(t, x, \xi)-x \cdot \xi+t p(x, \xi)) .
$$

$\Psi(t, x, \xi)$ clearly satisfies the statement of Proposition 5.5 . 
Using the phase function constructed in Proposition [5.5, we can define the FIO, $J(\Psi, a)$ : $\mathcal{S} \rightarrow \mathcal{S}^{\prime}$ by

$$
J(\Psi, a) f(x)=\frac{1}{(2 \pi)^{d}} \int e^{i(\Psi(t, x, \xi)-y \cdot \xi)} a(x, \xi) f(y) d y d \xi, \quad f \in \mathcal{S}\left(\mathbb{R}^{d}\right),
$$

where $a \in S(1, g)$. Moreover, we have the following.

Lemma 5.6. Let $t_{\varepsilon}>0$ be small enough. Then, for any bounded family of symbols $\{a(t) ;|t|<$ $\left.t_{\varepsilon}\right\} \subset S(1, g), J(\Psi, a)$ is bounded on $L^{2}\left(\mathbb{R}^{d}\right)$ uniformly with respect to $|t|<t_{\varepsilon}$ :

$$
\sup _{|t| \leq t_{\varepsilon}}\|J(\Psi, a)\|_{L^{2} \rightarrow L^{2}} \leq C_{\varepsilon}
$$

Proof. For sufficiently small $t_{\varepsilon}>0$, the estimates (5.6) imply

$$
\left|\left(\partial_{\xi} \otimes \partial_{x} \Psi\right)(t, x, \xi)-\mathrm{Id}\right| \leq C_{\varepsilon} t_{\varepsilon}<1 / 2, \quad\left|\partial_{x}^{\alpha} \partial_{\xi}^{\beta} \Psi(t, x, \xi)\right| \leq C_{\alpha \beta \varepsilon} \text { for }|\alpha+\beta| \geq 2,
$$

uniformly with respect to $(t, x, \xi) \in\left(-t_{\varepsilon}, t_{\varepsilon}\right) \times \mathbb{R}^{2 d}$. Therefore, the assertion is a consequence of the standard $L^{2}$-boundedness of FIO, or equivalently Kuranishi's trick and the $L^{2}$-boundedness of PDO (see, e.g., [26] or [24, Lemma 4.2]).

We next construct the amplitude.

Proposition 5.7. Let $\Psi(t, x, \xi)$ be as in Proposition 5.5 with $\varepsilon$ replaced by $\varepsilon / 3$. Then, for any integer $N \geq 0$, there exist families of symbols $\left\{b_{j}(t, \cdot, \cdot) ;|t|<t_{\varepsilon}\right\} \subset S\left(\langle x\rangle^{-j}\langle\xi\rangle^{-j}, g\right), j=$ $0,1,2, \ldots, N-1$, such that $\operatorname{supp} b_{j}(t, \cdot, \cdot) \subset \Omega(\varepsilon / 2)$ and $b_{j}$ solve the transport equations (5.5).

Proof. We consider the case $t \geq 0$ only. Symbols $b_{j}$ can be constructed by a standard method of characteristics, along the flow generated by $X(t, x, \xi)$, as follows. At first note that Assumption 1.1 and (5.6) imply that

$$
\begin{aligned}
\left|\partial_{x}^{\alpha} \partial_{\xi}^{\beta} X(t, x, \xi)\right| & \leq C_{\alpha \beta \varepsilon}\langle x\rangle^{1-|\alpha+\beta|}, \\
\left|\partial_{x}^{\alpha} \partial_{\xi}^{\beta} y(t, x, \xi)\right| & \leq C_{\alpha \beta \varepsilon}\langle x\rangle^{-|\alpha+\beta|},
\end{aligned}
$$

uniformly with respect to $0 \leq t \leq t_{\varepsilon}$ and $(x, \xi) \in \Omega(\varepsilon / 3)$. For all $0 \leq s, t \leq t_{\varepsilon}$, we consider the solution to the following ODE:

$$
\partial_{t} z(t, s, x, \xi)=X(t, z(t, s, x, \xi), \xi) ; \quad z(s, s)=x .
$$

We learn by (5.7) and a same argument as that in the proof of Lemma 5.1 that $z(t, s)$ is well defined for $0 \leq s, t \leq t_{\varepsilon}$ and $(x, \xi) \in \Omega(\varepsilon / 3)$, and that

$$
\left|\partial_{x}^{\alpha} \partial_{\xi}^{\beta}(z(t, s, x, \xi)-x)\right| \leq C_{\alpha \beta \varepsilon} t_{\varepsilon}\langle x\rangle^{1-|\alpha+\beta|}, \quad(x, \xi) \in \Omega(\varepsilon / 3) .
$$

Then, $b_{j}(t)$ are defined inductively by

$$
\begin{aligned}
& b_{0}(t, x, \xi)=\chi_{\varepsilon}^{*}(z(0, t, x, \xi), \xi) \exp \left(\int_{0}^{t} y(s, z(s, t, x, \xi), \xi) d s\right) \\
& b_{j}(t, x, \xi)=-\int_{0}^{t}\left(i K b_{j-1}\right)(s, z(s, t, x, \xi), \xi) \exp \left(\int_{u}^{t} y(u, z(u, t, x, \xi), \xi) d u\right) d s .
\end{aligned}
$$


Since $\operatorname{supp} \chi_{\varepsilon}^{*} \subset \Omega(\varepsilon)$, by (5.9) and a same argument as that in the proof of Lemma 5.2 (1), we see that $b_{j}(t, x, \xi)$ is smooth with respect to $(x, \xi)$ and that $\partial_{x}^{\alpha} \partial_{\xi}^{\beta} b_{j}(t, x, \xi)$ are supported in $\Omega(\varepsilon / 2)$ for all $0 \leq t \leq t_{\varepsilon}$. Thus, if we extend $b_{j}$ on $\mathbb{R}^{2 d}$ so that $b_{j}(t, x, \xi)=0$ outside $\Omega(\varepsilon / 2)$, then $b_{j}$ is still smooth in $(x, \xi)$. Furthermore, we learn by (5.8) and (5.9) that $\left\{b_{j}(t, \cdot, \cdot) ; t \in\right.$ $\left.\left[0, t_{\varepsilon}\right], 0 \leq j \leq N-1\right\}$ is a bounded set in $S\left(\langle x\rangle^{-j}\langle\xi\rangle^{-j}, g\right)$. Finally, a standard Hamilton-Jacobi theory shows that $b_{j}(t)$ solve the transport equations (5.5).

We now state the main result in this section.

Theorem 5.8. Let us fix $\varepsilon>0$ arbitrarily. Then, for sufficiently small $t_{\varepsilon}>0$, any nonnegative integer $N \geq 0$ and any symbol $\chi_{\varepsilon} \in S(1, g)$ supported in $\Omega(\varepsilon)$, we can find a bounded family of symbols $\left\{a^{N}(t, \cdot, \cdot) ;|t|<t_{\varepsilon}\right\} \subset S(1, g)$ such that $\mathrm{Op}\left(\chi_{\varepsilon}\right) e^{-i t H} \mathrm{Op}\left(\chi_{\varepsilon}\right)^{*}$ can be brought to the form

$$
\mathrm{Op}\left(\chi_{\varepsilon}\right) e^{-i t H} \mathrm{Op}\left(\chi_{\varepsilon}\right)^{*}=J\left(\Psi, a^{N}\right)+Q(t, N)
$$

where $J\left(\Psi, a^{N}\right)$ is the FIO with the phase $\Psi(t, x, \xi)$ constructed in Proposition 5.5 with $\varepsilon$ replaced by $\varepsilon / 3$. The distribution kernel of $J\left(\Psi, a^{N}\right)$, which we denote by $K_{\Psi, a^{N}}(t, x, y)$, satisfies the dispersive estimate:

$$
\left|K_{\Psi, a^{N}}(t, x, y)\right| \leq C_{N, \varepsilon}|t|^{-d / 2}, \quad 0<|t|<t_{\varepsilon}, x, \xi \in \mathbb{R}^{d} .
$$

Moreover, for any $\gamma \geq 0$ with $N>2 \gamma$, the remainder $Q(t, N)$ satisfies

$$
\left\|\langle D\rangle^{\gamma} Q(t, N)\langle D\rangle^{\gamma}\right\|_{L^{2} \rightarrow L^{2}} \leq C_{N \gamma \varepsilon}|t|, \quad|t|<t_{\varepsilon}
$$

In particular, if we choose $N \geq d+1$, then the distribution kernel of $Q(t, N)$ is uniformly bounded in $\mathbb{R}^{2 d}$ with respect to $|t|<t_{\varepsilon}$, and hence

$$
\left\|\mathrm{Op}\left(\chi_{\varepsilon}\right) e^{-i t H} \mathrm{Op}\left(\chi_{\varepsilon}\right)^{*}\right\|_{L^{1} \rightarrow L^{\infty}} \leq C_{\varepsilon}|t|^{-d / 2}, \quad 0<|t|<t_{\varepsilon} .
$$

Proof. We consider the case when $t \geq 0$ and the proof for the opposite case is similar. By virtue of Lemma 5.4, we may replace $\mathrm{Op}\left(\chi_{\varepsilon}\right)^{*}$ by $\mathrm{Op}\left(\chi_{\varepsilon}^{*}\right)$ for some $\chi_{\varepsilon}^{*} \in S(1, g)$ supported in $\Omega(\varepsilon)$, without loss of generality. Let $b^{N}=\sum_{j=0}^{N-1} b_{j}$ with $b_{j}$ constructed in Proposition 5.7. Since $\left.J\left(\Psi, b^{N}\right)\right|_{t=0}=\operatorname{Op}\left(\chi_{\varepsilon}^{*}\right)$, we have the Duhamel formula

$$
\mathrm{Op}\left(\chi_{\varepsilon}\right) e^{-i t H} \mathrm{Op}\left(\chi_{\varepsilon}^{*}\right)=\operatorname{Op}\left(\chi_{\varepsilon}\right) J\left(\Psi, b^{N}\right)-\left.i \int_{0}^{t} \mathrm{Op}\left(\chi_{\varepsilon}\right) e^{-i(t-s) H}\left(D_{t}+H\right) J\left(\Psi, b^{N}\right)\right|_{t=s} d s .
$$

Estimates on the remainder. It suffices to show that

$$
\sup _{|t|<t_{\varepsilon}}\left\|\langle D\rangle^{\gamma} \operatorname{Op}\left(\chi_{\varepsilon}\right) e^{-i t H}\left(D_{t}+H\right) J\left(\Psi, b^{N}\right)\langle D\rangle^{\gamma}\right\|_{L^{2} \rightarrow L^{2}} \leq C_{N \gamma \varepsilon} .
$$

Since $\Psi, b_{j}$ solve the Hamilton-Jacobi equation (5.4) and transport equations (5.5), respectively, a direct computation yields

$$
e^{-i \Psi(t, x, \xi)}\left(D_{t}+H\right)\left(e^{i \Psi(t, x, \xi)} \sum_{j=0}^{N-1} b_{j}(t, x, \xi)\right)=r_{N}(t, x, \xi),
$$


with some $\left\{r_{N}(t, \cdot, \cdot) ; 0 \leq t \leq t_{\varepsilon}\right\} \subset S\left(\langle x\rangle^{-N}\langle\xi\rangle^{-N}, g\right)$. In particular,

$$
\left(D_{t}+H\right) J\left(\Psi, b^{N}\right)=J\left(\Psi, r_{N}\right)
$$

A standard $L^{2}$-boundedness of FIO then implies

$$
\sup _{|t|<t_{\varepsilon}}\left\|\langle x\rangle^{\gamma+\delta}\langle D\rangle^{\gamma+\delta} J\left(\Psi, r_{N}\right)\langle D\rangle^{\gamma}\right\|_{L^{2} \rightarrow L^{2}} \leq C_{N \gamma \delta}
$$

for any $\gamma, \delta \geq 0$ with $2 \gamma+\delta \leq N$. Since we already proved in the proof of Lemma 5.4 that

$$
\sup _{|t| \leq t_{\varepsilon}}\left\|\langle D\rangle^{\gamma} \mathrm{Op}\left(\chi_{\varepsilon}\right) e^{-i t H}\langle D\rangle^{-\gamma-\delta}\langle x\rangle^{-\gamma-\delta}\right\|_{L^{2} \rightarrow L^{2}} \leq C_{\gamma \delta},
$$

we obtain the desired estimate.

Dispersive estimates. By the composition formula of PDO and FIO (cf. [26]), Op $\left(\chi_{\varepsilon}\right) J\left(\Psi, b^{N}\right)$ is also a FIO with the same phase $\Psi$ and the amplitude

$$
a^{N}(t, x, \xi)=\frac{1}{(2 \pi)^{d}} \int e^{i y \cdot \eta} \chi_{\varepsilon}(x, \eta+\widetilde{\Xi}(t, x, y, \xi)) b^{N}(t, x+y, \xi) d y d \eta
$$

where $\widetilde{\Xi}(t, x, y, \xi)=\int_{0}^{1}\left(\partial_{x} \Psi\right)(t, y+\lambda(x-y), \xi) d \lambda$. By virtue of ([5.6), $\widetilde{\Xi}$ satisfies

$$
\left|\partial_{x}^{\alpha} \partial_{y}^{\alpha^{\prime}} \partial_{\xi}^{\beta}(\widetilde{\Xi}(t, x, y, \xi)-\xi)\right| \leq C_{\alpha \alpha^{\prime} \beta}|t|, \quad\left|\alpha+\alpha^{\prime}+\beta\right| \geq 1 .
$$

Combining with the fact that $\chi_{\varepsilon}, b^{N} \in S(1, g), \operatorname{supp} \chi_{\varepsilon} \subset \Omega(\varepsilon)$ and $\operatorname{supp} b^{N}(t, \cdot, \cdot) \subset \Omega(\varepsilon / 2)$, we see that $\left\{a^{N} ; 0 \leq t<t_{\varepsilon}\right\}$ is bounded in $S(1, g)$. The distribution kernel of $J\left(\Psi, a^{N}\right)$ is given by

$$
K_{\Psi, a^{N}}(t, x, y)=\frac{1}{(2 \pi)^{d}} \int e^{i(\Psi(t, x, \xi)-y \cdot \xi)} a^{N}(t, x, \xi) d \xi .
$$

By virtue of Proposition 5.5, we have

$$
\begin{aligned}
& \sup _{|t| \leq t_{\varepsilon}}\left|\partial_{x}^{\alpha} \partial_{y}^{\beta} \partial_{\xi}^{\gamma}(\Psi(t, x, \xi)-y \cdot \xi)\right| \leq C_{\alpha \beta \gamma}, \quad|\alpha+\beta+\gamma| \geq 2, \\
& \partial_{\xi}^{2} \Psi(t, x, \xi)=-t\left(g^{j k}(x)\right)_{j, k}+O\left(t^{2}\right), \quad|t| \rightarrow 0 .
\end{aligned}
$$

As a consequence, since $g^{j k}(x)$ is uniformly elliptic, the phase function $\Psi(t, x, \xi)-y \cdot \xi$ has a unique non-degenerate critical point for all $|t|<t_{\varepsilon}$ and we can apply the stationary phase method to $K_{\Psi, a^{N}}(t, x, y)$, provided that $t_{\varepsilon}>0$ is small enough. Therefore,

$$
\left|K_{\Psi, a^{N}}(t, x, y)\right| \leq C|t|^{-d / 2}, \quad 0<|t| \leq t_{\varepsilon}, x, \xi \in \mathbb{R}^{d},
$$

which completes the proof.

\section{Proof of Theorem 1.5}

Suppose that $H$ satisfies Assumption 1.1 with $\mu \geq 0$. In this section we give the proof of Theorem 1.5. In view of Corollary 2.6, (1.4) is a consequence of the following proposition. 
Proposition 6.1. For any symbol $a \in C_{0}^{\infty}\left(\mathbb{R}^{2 d}\right)$ and $T>0$,

$$
\left\|\mathrm{Op}_{h}(a) e^{-i t H} \varphi\right\|_{L^{p}\left([-T, T] ; L^{q}\left(\mathbb{R}^{d}\right)\right)} \leq C_{T} h^{-1 / p}\|\varphi\|_{L^{2}\left(\mathbb{R}^{d}\right)},
$$

uniformly with respect to $h \in(0,1]$, provided that $(p, q)$ satisfies (1.1).

Proof. This proposition follows from the standard WKB approximation for $e^{-i t H} \mathrm{Op}_{h}(a)$ up to time scales of order $1 / h$. The proof is essentially same as that in the case for the LaplaceBeltrami operator on compact manifolds without boundaries (see, [6, Section 2]), and we omit details.

Using this proposition, we have the semiclassical Strichartz estimates with inhomogeneous error terms:

Proposition 6.2. Let $a \in C_{0}^{\infty}\left(\mathbb{R}^{2 d}\right)$. Then, for any $T>0$ and any $(p, q)$ satisfying the admissible condition (1.1),

$$
\begin{aligned}
\left\|\mathrm{Op}_{h}(a) e^{-i t H} \varphi\right\|_{L^{p}\left([-T, T] ; L^{q}\left(\mathbb{R}^{d}\right)\right)} & \leq C_{T}\left\|\mathrm{Op}_{h}(a) \varphi\right\|_{L^{2}\left(\mathbb{R}^{d}\right)}+C_{T} h\|\varphi\|_{L^{2}\left(\mathbb{R}^{d}\right)} \\
& +C h^{-1 / 2}\left\|\mathrm{Op}_{h}(a) e^{-i t H} \varphi\right\|_{L^{2}\left([-T, T] ; L^{2}\left(\mathbb{R}^{d}\right)\right)} \\
& +C h^{1 / 2}\left\|\left[\mathrm{Op}_{h}(a), H\right] e^{-i t H} \varphi\right\|_{L^{2}\left([-T, T] ; L^{2}\left(\mathbb{R}^{d}\right)\right)}
\end{aligned}
$$

uniformly with respect to $h \in(0,1]$.

This proposition has been proved by [4] for the case with $V, A \equiv 0$. We give a refinement of this proposition with its proof in Section 7 .

Next, we shall prove that if $k(x, \xi)$ satisfies the nontrapping condition (1.3), then the missing $1 / p$ derivative can be recovered. We first recall the local smoothing effects for Schrödinger operators proved by Doi [11. For any $s \in \mathbb{R}$, we set $\mathcal{B}^{s}:=\left\{f \in L^{2}\left(\mathbb{R}^{d}\right) ;\langle x\rangle^{s} f,\langle D\rangle^{s} f \in L^{2}\left(\mathbb{R}^{d}\right)\right\}$. Define a symbol $e_{s}(x, \xi)$ by

$$
e_{s}(x, \xi):=\left(k(x, \xi)+|x|^{2}+L(s)\right)^{s / 2} \in S\left((1+|x|+|\xi|)^{s}, g\right),
$$

where $L(s)>1$ is a large constant depending on $s$. We denote by $E_{s}$ its Weyl quantization:

$$
E_{s} f(x)=\mathrm{Op}^{w}\left(e_{s}\right) f(x)=\frac{1}{(2 \pi)^{d}} \int e^{i(x-y) \cdot \xi} e_{s}\left(\frac{x+y}{2}, \xi\right) f(y) d y d \xi
$$

Then, for any $s \in \mathbb{R}$, there exists $L(s)>0$ such that $E_{s}$ is a homeomorphism from $\mathcal{B}^{r+s}$ to $\mathcal{B}^{r}$ for all $r \in \mathbb{R}$, and $\left(E_{s}\right)^{-1}$ is still a Weyl quantization of a symbol in $S\left((1+|x|+|\xi|)^{-s}, g\right.$ ) (see, [11, Lemma 4.1]).

Proposition 6.3 (The local smoothing effects [11]). Suppose that $k(x, \xi)$ satisfies the nontrapping condition (1.3) and that Assumption 1.4. Then, for any $T>0$ and $\sigma>0$, there exists $C_{T, \sigma}>0$ such that

$$
\left\|\langle x\rangle^{-1 / 2-\sigma} E_{1 / 2} e^{-i t H} \varphi\right\|_{L^{2}\left([-T, T] ; L^{2}\left(\mathbb{R}^{d}\right)\right)} \leq C_{T, \sigma}\|\varphi\|_{L^{2}\left(\mathbb{R}^{d}\right)} .
$$


Remark 6.4. (6.1) implies a standard local smoothing effect:

$$
\left\|\langle x\rangle^{-1 / 2-\sigma}\langle D\rangle^{1 / 2} e^{-i t H} \varphi\right\|_{L^{2}\left([-T, T] ; L^{2}\left(\mathbb{R}^{d}\right)\right)} \leq C_{T, \sigma}\|\varphi\|_{L^{2}\left(\mathbb{R}^{d}\right)} .
$$

Indeed, we compute

$$
\begin{aligned}
& \langle x\rangle^{-1 / 2-\sigma}\langle D\rangle^{1 / 2} \\
& =\langle D\rangle^{1 / 2}\langle x\rangle^{-1 / 2-\sigma}+\left[\langle D\rangle^{1 / 2},\langle x\rangle^{-1 / 2-\sigma}\right] \\
& =\langle D\rangle^{1 / 2}\left(E_{1 / 2}\right)^{-1} E_{1 / 2}\langle x\rangle^{-1 / 2-\sigma}+\left[\langle D\rangle^{1 / 2},\langle x\rangle^{-1 / 2-\sigma}\right] \\
& =\langle D\rangle^{1 / 2}\left(E_{1 / 2}\right)^{-1}\left(\langle x\rangle^{-1 / 2-\sigma} E_{1 / 2}+\left[E_{1 / 2},\langle x\rangle^{-1 / 2-\sigma}\right]\right)+\left[\langle D\rangle^{1 / 2},\langle x\rangle^{-1 / 2-\sigma}\right] .
\end{aligned}
$$

It is easy to see that $\langle D\rangle^{1 / 2}\left(E_{1 / 2}\right)^{-1},\left[E_{1 / 2},\langle x\rangle^{-1 / 2-\sigma}\right]$ and $\left[\langle D\rangle^{1 / 2},\langle x\rangle^{-1 / 2-\sigma}\right]$ are bounded on $L^{2}\left(\mathbb{R}^{d}\right)$ since their symbols belong to $S(1, g)$. Therefore, (6.1) implies (6.2).

Proof of (1.5) of Theorem 1.5. It is clear that (1.5) follows from Proposition 6.2. (6.2) and Corollary 2.6, since $a$ is compactly supported with respect to $x$ and $\{a, p\} \in S(\langle\xi\rangle, g)$, where $p=p(x, \xi)$.

\section{$7 \quad$ Near sharp Strichartz estimates without asymptotic flatness}

This section is devoted to prove Theorem 1.6. We may assume $\mu=0$ without loss of generality. We begin with the following proposition.

Proposition 7.1. Let $I \Subset(0, \infty)$ be a relatively compact open interval and $C_{0}>1$. Then, there exist $\delta_{0}, h_{0}>0$ such that for any $0<\delta \leq \delta_{0}, 0<h \leq h_{0}, 1 \leq R \leq 1 / h$ and any symbol $a_{h} \in S(1, g)$ supported in $\left\{(x, \xi) ; R<|x|<C_{0} / h,|\xi| \in I\right\}$, we have

$$
\left\|\mathrm{Op}_{h}\left(a_{h}\right) e^{-i t H} \mathrm{Op}_{h}\left(a_{h}\right)^{*}\right\|_{L^{1} \rightarrow L^{\infty}} \leq C_{\delta}|t|^{-d / 2}, \quad 0<|t|<\delta h R,
$$

where $C_{\delta}>0$ may be taken uniformly with respect to $h$ and $R$.

Remark 7.2. When $|t|>0$ in (7.1) is small and independent of $R$, Proposition 7.1 is wellknown and the proof is given by the standard method of the short-time WKB approximation for $e^{-i t H^{h} / h} \mathrm{Op}_{h}\left(a_{h}\right)^{*}$ (see, e.g., [6]).

For $h \in(0,1], R \geq 1$, an open interval $I \Subset(0, \infty)$ and $C_{0}>1$, we set

$$
\Gamma(R, h, I):=\left\{(x, \xi) \in \mathbb{R}^{2 d} ; R<|x|<C_{0} / h,|\xi| \in I\right\} .
$$

Proposition 7.1 is a consequence of the same argument as in the proof of Proposition 3.1 and the following proposition:

Proposition 7.3. Let $I \Subset I_{1} \Subset(0, \infty)$ and $C_{0}>1$. Then, there exist $\delta_{0}, h_{0}>0$ such that the followings hold for any $0<\delta \leq \delta_{0}, 0<h \leq h_{0}$ and $1 \leq R \leq C_{0} / h$.

(1) There exists $\Phi_{h}(t, x, \xi) \in C^{\infty}\left((-\delta R, \delta R) \times \mathbb{R}^{2 d}\right)$ such that $\Phi_{h}$ solves the following HamiltonJacobi equation:

$$
\left\{\begin{aligned}
\partial_{t} \Phi_{h}(t, x, \xi) & =-p_{h}\left(x, \partial_{x} \Phi_{h}(t, x, \xi)\right), \quad|t|<\delta R, \quad(x, \xi) \in \Gamma\left(R / 2, h / 2, I_{1}\right) \\
\Phi_{h}(0, x, \xi) & =x \cdot \xi, \quad(x, \xi) \in \Gamma\left(R / 2, h / 2, I_{1}\right)
\end{aligned}\right.
$$


Furthermore, we have

$$
\left|\partial_{x}^{\alpha} \partial_{\xi}^{\beta}\left(\Phi_{h}(t, x, \xi)-x \cdot \xi+t p_{h}(x, \xi)\right)\right| \leq C_{\alpha \beta} R^{-|\alpha|} h|t|^{2}, \quad \alpha, \beta \in \mathbb{Z}_{+}^{d},
$$

uniformly with respect to $x, \xi \in \mathbb{R}^{d}, h \in\left(0, h_{0}\right], 0 \leq R \leq C_{0} / h$ and $|t|<\delta R$.

(2) For any $a_{h} \in S(1, g)$ with $\operatorname{supp} a_{h} \subset \Gamma(R, h, I)$ and any integer $N \geq 0$, we can find $b_{h}^{N}(t, \cdot, \cdot) \in$ $S(1, g)$ such that

$$
e^{-i t \widetilde{H}^{h} / h} \mathrm{Op}_{h}\left(a_{h}\right)^{*}=J_{h}\left(\Phi_{h}, b_{h}^{N}\right)+Q_{\mathrm{WKB}}(t, h, N),
$$

where $J_{h}\left(\Phi_{h}, b_{h}^{N}\right)$ is the $h$-FIO with the phase function $\Phi_{h}$ and the amplitude $b_{h}^{N}$, and its distribution kernel satisfies

$$
\left|K_{\mathrm{WKB}}(t, h, x, y)\right| \leq C|t h|^{-d / 2}, \quad h \in\left(0, h_{0}\right], 0<|t| \leq \delta R, x, \xi \in \mathbb{R}^{d} .
$$

Moreover the remainder $Q_{\mathrm{WKB}}(t, h, N)$ satisfies

$$
\left\|\langle D\rangle^{s} Q_{\mathrm{WKB}}(t, h, N)\langle D\rangle^{s}\right\|_{L^{2} \rightarrow L^{2}} \leq C_{N, s} h^{N-2 s}|t|, \quad h \in\left(0, h_{0}\right],|t| \leq \delta R .
$$

Sketch of the proof. The proof is similar to that of Theorem 5.8 and, in particular, the proof of the second claim is completely same. Thus, we give only the outline of the construction of $\Phi_{h}$. We may assume $C_{0}=1$ without loss of generality. Let us denote by $\left(X_{h}, \Xi_{h}\right)$ the Hamilton flow generated by $p_{h}$. To construct the phase function, the most important step is to study the inverse map of $(x, \xi) \mapsto\left(X_{h}(t, x, \xi), \xi\right)$. Choose an open interval $\widetilde{I}_{1}$ so that $I_{1} \Subset \widetilde{I}_{1} \Subset(0, \infty)$. The following bounds have been proved by [24]:

$$
\left|\partial_{x}^{\alpha} \partial_{\xi}^{\beta}\left(X_{h}(t, x, \xi)-x\right)\right|+\langle x\rangle\left|\partial_{x}^{\alpha} \partial_{\xi}^{\beta}\left(\Xi_{h}(t, x, \xi)-\xi\right)\right| \leq C_{\alpha \beta}\langle x\rangle^{-|\alpha|}|t|,
$$

for $(x, \xi) \in \Gamma\left(R / 3, h / 3, \widetilde{I}_{1}\right)$ and $|t| \leq \delta R$. For sufficiently small $\delta>0$ and for any fixed $|t| \leq \delta R$, the above estimates imply

$$
\left|\partial_{x} X_{h}(t)-\mathrm{Id}\right| \leq C R^{-1}|t| \leq C \delta<1 / 2
$$

By the same argument as that in the proof of Lemma 5.2, the map $(x, \xi) \mapsto\left(X_{h}(t, x, \xi), \xi\right)$ is a diffeomorphism from $\Gamma\left(R / 3, h / 3, \widetilde{I}_{1}\right)$ onto its range and the corresponding inverse $(x, \xi) \mapsto$ $\left(Y_{h}(t, x, \xi), \xi\right)$ is well-defined for $|t|<\delta R$ and $(x, \xi) \in \Gamma\left(R / 2, h / 2, I_{1}\right)$. Moreover, $Y_{h}(t)$ satisfies the same estimates as that for $X_{h}(t)$ :

$$
\left|\partial_{x}^{\alpha} \partial_{\xi}^{\beta}\left(Y_{h}(t, x, \xi)-x\right)\right| \leq C_{\alpha \beta}\langle x\rangle^{-|\alpha|}|t|, \quad|t|<\delta R,(x, \xi) \in \Gamma\left(R / 2, h / 2, I_{1}\right) .
$$

We now define $\Phi_{h}$ by

$$
\Phi_{h}(t, x, \xi):=x \cdot \xi+\int_{0}^{t} L_{h}\left(X_{h}(s, Y(t, x, \xi), \xi), \Xi(s, y(t, x, \xi), \xi)\right) d s,
$$

where $L_{h}=\xi \cdot \partial_{\xi} p_{h}-p_{h}$. By the standard Hamilton-Jacobi theory, $\Phi_{h}$ solves (7.2). Moreover, using the energy conservation $p_{h}\left(x, \partial_{x} \Phi_{h}(t)\right)=p_{h}\left(Y_{h}(t), \xi\right)$ and the above estimates on $X_{h}, \Xi_{h}$ 
and $Y_{h}$, we see that

$$
\begin{aligned}
\left|p_{h}\left(x, \partial_{x} \Phi_{h}(t)\right)-p_{h}(x, \xi)\right| & =\left|p_{h}\left(Y_{h}(t), \xi\right)-p_{h}(x, \xi)\right| \\
& \leq\left|Y_{h}(t)-x\right|\left|\int_{0}^{\lambda}\left(\partial_{x} p_{h}\right)\left(\lambda Y_{h}(t)-(1-\lambda) x, \xi\right) d \lambda\right| \\
& \leq C|y(t)-x|\left(h+h^{2}\langle x\rangle^{2}\right) \\
& \leq C h|t|,
\end{aligned}
$$

and that

$$
\left|\partial_{x}^{\alpha} \partial_{\xi}^{\beta}\left(p_{h}\left(x, \partial_{x} \Phi_{h}\right)-p_{h}(x, \xi)\right)\right| \leq C_{\alpha \beta}\langle x\rangle^{-|\alpha|} h|t| .
$$

Using these estimates, we can check that $\Phi_{h}$ satisfies (7.3). Finally, we extend $\Phi_{h}$ to the whole space so that $\Phi_{h}(t, x, \xi)=x \cdot \xi-t p_{h}(x, \xi)$ outside $\Gamma\left(R / 3, h / 3, \widetilde{I}_{1}\right)$.

Using Proposition 7.1, we obtain a refinement of Proposition 6.2.

Proposition 7.4. Let $0<R \leq 1 / h$ and let $a_{h} \in S(1, g)$ be supported in $\{(x, \xi) ; R<|x|<$ $1 / h,|\xi| \in I\}$. Then, for any $T>0$ and $(p, q)$ satisfying the admissible condition (1.1),

$$
\begin{aligned}
\left\|\mathrm{Op}_{h}\left(a_{h}\right) e^{-i t H} \varphi\right\|_{L^{p}\left([-T, T] ; L^{q}\left(\mathbb{R}^{d}\right)\right)} & \leq C_{T}\left\|\mathrm{Op}_{h}\left(a_{h}\right) \varphi\right\|_{L^{2}\left(\mathbb{R}^{d}\right)}+C_{T} h\|\varphi\|_{L^{2}\left(\mathbb{R}^{d}\right)} \\
& +C_{T}(h R)^{-1 / 2}\left\|\mathrm{Op}_{h}\left(a_{h}\right) e^{-i t H} \varphi\right\|_{L^{2}\left([-T, T] ; L^{2}\left(\mathbb{R}^{d}\right)\right)} \\
& +C_{T}(h R)^{1 / 2}\left\|\left[H, \mathrm{Op}_{h}\left(a_{h}\right)\right] e^{-i t H} \varphi\right\|_{L^{2}\left([-T, T] ; L^{2}\left(\mathbb{R}^{d}\right)\right)},
\end{aligned}
$$

uniformly with respect to $h \in\left(0, h_{0}\right]$.

Proof. The proof is similar to that of [4, Proposition 5.4]. By time reversal invariance we can restrict our considerations to the interval $[0, T]$. We may assume $T \geq h R$ without loss of generality and split $[0, T]$ as follows: $[0, T]=J_{0} \cup J_{1} \cup \cdots \cup J_{N}$, where $J_{j}=[j h R,(j+1) h R]$, $0 \leq j \leq N-1$, and $J_{N}=[T-\delta h R, T]$. For $j=0$, we have the Duhamel formula

$$
\mathrm{Op}_{h}\left(a_{h}\right) e^{-i t H}=e^{-i t H} \mathrm{Op}_{h}\left(a_{h}\right)-i \int_{0}^{t} e^{-i(t-s) H}\left[\mathrm{Op}_{h}\left(a_{h}\right), H\right] e^{-i s H} d s, \quad t \in J_{0} .
$$

We here choose $b_{h} \in S(1, g)$ so that $b_{h} \equiv 1$ on $\operatorname{supp} a$ and $b_{h}$ is supported in a sufficiently small neighborhood of $\operatorname{supp} a_{h}$. By Proposition 7.1, $\mathrm{Op}_{h}\left(b_{h}\right) e^{-i(t-s) H} \mathrm{Op}_{h}\left(b_{h}\right)^{*}$ satisfies dispersive estimates (7.1) for $0<|t-s|<\delta h R$ with some $\delta>0$ small enough. Using the Keel-Tao theorem [19] and the unitarity of $e^{-i t H}$, we then learn that for any interval $J_{R}$ of size $\left|J_{R}\right| \leq 2 h R$, the following homogeneous and inhomogeneous Strichartz estimates hold uniformly with respect to $h \in\left(0, h_{0}\right]:$

$$
\begin{gathered}
\left\|\mathrm{Op}_{h}\left(b_{h}\right) e^{-i t H} \varphi\right\|_{L^{p}\left(J_{R} ; L^{q}\left(\mathbb{R}^{d}\right)\right)} \leq C\|\varphi\|_{L^{2}\left(\mathbb{R}^{d}\right)}, \\
\left\|\int_{0}^{t} F\left(s \in J_{R}\right) \mathrm{Op}_{h}\left(b_{h}\right) e^{-i(t-s) H} \mathrm{Op}_{h}\left(b_{h}\right)^{*} g(s) d s\right\|_{L^{p}\left(J_{R} ; L^{q}\left(\mathbb{R}^{d}\right)\right)} \leq C\|g\|_{L^{1}\left(J_{R} ; L^{2}\left(\mathbb{R}^{d}\right)\right)},
\end{gathered}
$$

where $F\left(s \in J_{R}\right)$ is the characteristic function of $J_{R}$ and $(p, q)$ satisfies the admissible condition (1.1). On the other hand, using the expansions (2.3) and (2.4), we see that for any $M \geq 0$,

$$
\begin{aligned}
\mathrm{Op}_{h}\left(a_{h}\right) & =\mathrm{Op}_{h}\left(b_{h}\right) \mathrm{Op}_{h}\left(a_{h}\right)+h^{M} \mathrm{Op}_{h}\left(r_{1, h}\right) \\
& =\mathrm{Op}_{h}\left(b_{h}\right)^{*} \operatorname{Op}_{h}\left(a_{h}\right)+h^{M} \mathrm{Op}_{h}\left(r_{2, h}\right), \\
{\left[\mathrm{Op}_{h}\left(a_{h}\right), H\right] } & =\mathrm{Op}_{h}\left(b_{h}\right)^{*}\left[\mathrm{Op}_{h}\left(a_{h}\right), H\right]+h^{M} \mathrm{Op}_{h}\left(r_{3, h}\right),
\end{aligned}
$$


with some $\left\{r_{l, h}\right\}_{h \in(0,1]}, l=1,2,3$, which are bounded in $S\left(\langle x\rangle^{-M}\langle\xi\rangle^{-M}, g\right)$. Therefore, we can write

$$
\begin{aligned}
\mathrm{Op}_{h}\left(a_{h}\right) e^{-i t H} & =\mathrm{Op}_{h}\left(b_{h}\right) e^{-i t H} \mathrm{Op}_{h}\left(a_{h}\right) \\
& -i \int_{0}^{t} \mathrm{Op}_{h}\left(b_{h}\right) e^{-i(t-s) H} \mathrm{Op}_{h}\left(b_{h}\right)^{*}\left[\mathrm{Op}_{h}\left(a_{h}\right), H\right] e^{-i s H} d s \\
& +Q(t, h, M),
\end{aligned}
$$

where the remainder $Q(t, h, M)$ satisfies

$$
\|Q(t, h, M)\|_{L^{2} \rightarrow L^{q}} \leq C_{M} h^{M-1-d(1 / 2-1 / q)}, \quad 2 \leq q \leq \infty,
$$

uniformly in $h \in(0,1]$. Combining this estimate with (7.5) and (7.6), we obtain

$$
\begin{aligned}
& \left\|\mathrm{Op}_{h}\left(a_{h}\right) e^{-i t H}\right\|_{L^{p}\left(J_{0} ; L^{q}\right)} \\
& \leq C\left\|\mathrm{Op}_{h}\left(a_{h}\right) \varphi\right\|_{L^{2}}+C h\|\varphi\|_{L^{2}}+C\left\|\left[\mathrm{Op}_{h}\left(a_{h}\right), H\right] e^{-i t H} \varphi\right\|_{L^{1}\left(J_{0} ; L^{2}\right)} \\
& \leq C\left\|\mathrm{Op}_{h}\left(a_{h}\right) \varphi\right\|_{L^{2}}+C h\|\varphi\|_{L^{2}}+C(h R)^{1 / 2}\left\|\left[\mathrm{Op}_{h}\left(a_{h}\right), H\right] e^{-i t H} \varphi\right\|_{L^{2}\left(J_{0} ; L^{2}\right)}
\end{aligned}
$$

We similarly obtain the same bound for $j=N$ :

$$
\begin{aligned}
& \left\|\mathrm{Op}_{h}\left(a_{h}\right) e^{-i t H}\right\|_{L^{p}\left(J_{N} ; L^{q}\right)} \\
& \leq C\left\|\mathrm{Op}_{h}\left(a_{h}\right) \varphi\right\|_{L^{2}}+C h\|\varphi\|_{L^{2}}+C(h R)^{1 / 2}\left\|\left[\mathrm{Op}_{h}\left(a_{h}\right), H\right] e^{-i t H} \varphi\right\|_{L^{2}\left(J_{N} ; L^{2}\right)} .
\end{aligned}
$$

For $j=1,2, \ldots, N-1$, taking $\theta \in C_{0}^{\infty}(\mathbb{R})$ so that $\theta \equiv 1$ on $[-1 / 2,1 / 2]$ and $\operatorname{supp} \theta \subset[-1,1]$, we set $\left.\theta_{j}(t)=\theta(t /(h R)-j-1 / 2)\right)$. It is easy to see that $\theta_{j} \equiv 1$ on $J_{j}$ and $\operatorname{supp} \theta_{j} \subset \widetilde{J}_{j}=$ $J_{j}+[-h R / 2, h R / 2]$. We consider $v_{j}=\theta_{j}(t) \mathrm{Op}_{h}\left(a_{h}\right) e^{-i t H} \varphi$, which solves

$$
i \partial_{t} v_{j}=H v_{j}+\theta_{j}^{\prime} \mathrm{Op}_{h}\left(a_{h}\right) e^{-i t H} \varphi+\theta_{j}\left[\mathrm{Op}_{h}\left(a_{h}\right), H\right] e^{-i t H} \varphi ;\left.\quad v_{j}\right|_{t=0}=0 .
$$

A same argument as above and the Duhamel formula then imply that, for any $t \in \widetilde{J}_{j}$ and $M \geq 0$, $v_{j}$ satisfies

$$
\begin{aligned}
v_{j} & =-i \int_{0}^{t} \mathrm{Op}_{h}\left(b_{h}\right) e^{-i(t-s) H} \mathrm{Op}_{h}\left(b_{h}\right)^{*}\left(\theta_{j}^{\prime}(s) \mathrm{Op}_{h}\left(a_{h}\right)+\theta_{j}(s)\left[\mathrm{Op}_{h}\left(a_{h}\right), H\right]\right) e^{-i s H} \varphi d s \\
& +\widetilde{Q}(t, h, M),
\end{aligned}
$$

where the remainder $\widetilde{Q}(t, h, M)$ satisfies

$$
\|\widetilde{Q}(t, h, M)\|_{L^{2} \rightarrow L^{q}} \leq C_{M} h^{M-1-d(1 / 2-1 / q)}, \quad 2 \leq q \leq \infty,
$$

uniformly in $h \in(0,1]$ and $t \in \widetilde{J}_{j}$. Taking $M \geq 0$ large enough, we learn

$$
\begin{aligned}
& \left\|v_{j}\right\|_{L^{p}\left(J_{j} ; L^{q}\right)} \\
& \leq C h^{2}\|\varphi\|_{L^{2}}+C(h R)^{-1}\left\|\mathrm{Op}_{h}\left(a_{h}\right) e^{-i t H} \varphi\right\|_{L^{1}\left(\widetilde{J}_{j} ; L^{2}\right)}+C\left\|\left[\mathrm{Op}_{h}\left(a_{h}\right), H\right] e^{-i t H} \varphi\right\|_{L^{1}\left(\widetilde{J}_{j} ; L^{2}\right)} \\
& \leq C h^{2}\|\varphi\|_{L^{2}}+C(h R)^{-1 / 2}\left\|\mathrm{Op}_{h}\left(a_{h}\right) e^{-i t H} \varphi\right\|_{L^{2}\left(\widetilde{J}_{j} ; L^{2}\right)} \\
& \quad+C(h R)^{1 / 2}\left\|\left[\mathrm{Op}_{h}\left(a_{h}\right), H\right] e^{-i t H} \varphi\right\|_{L^{2}\left(\widetilde{J}_{j} ; L^{2}\right)}
\end{aligned}
$$

Summing over $j=0,1, \ldots, N$, since $N \leq T / h$ and $p \geq 2$, we have the assertion by Minkowski's inequality. 
Proof of Theorem 1.6. In view of Corollary 2.6, Theorem 1.5] and Proposition 3.2, it suffices to show that, for any $a_{h} \in S(1, g)$ with supp $a_{h} \in\{(x, \xi) ; 2 \leq|x| \leq 1 / h,|\xi| \in I\}$ and any $\varepsilon>0$,

$$
\sum_{h}\left\|\mathrm{Op}_{h}\left(a_{h}\right) e^{-i t H} f\left(h^{2} H\right) \varphi\right\|_{L^{p}\left([-T, T] ; L^{q}\right)}^{2} \leq C_{T, \varepsilon}\left\|\langle H\rangle^{\varepsilon} \varphi\right\|_{L^{2}}^{2} .
$$

Let us consider a dyadic partition of unity:

$$
\sum_{1 \leq j \leq j_{h}} \chi\left(2^{-j} x\right)=1, \quad 2 \leq|x| \leq 1 / h
$$

where $\chi \in C_{0}^{\infty}\left(\mathbb{R}^{d}\right)$ with $\operatorname{supp} \chi \subset\{1 / 2<|x|<2\}$ and $j_{h} \leq[\log (1 / h)]+1$. We set $\chi_{j}(x)=$ $\chi\left(2^{-j} x\right)$. Proposition 7.4 then implies

$$
\begin{aligned}
\left\|\chi_{j} \mathrm{Op}_{h}\left(a_{h}\right) e^{-i t H} \varphi\right\|_{L^{p}\left([-T, T] ; L^{q}\right)} & \leq C_{T}\left\|\chi_{j} \mathrm{Op}_{h}\left(a_{h}\right) \varphi\right\|_{L^{2}}+C_{T} h\|\varphi\|_{L^{2}} \\
& +C_{T}\left(h 2^{j}\right)^{-1 / 2}\left\|\chi_{j} \mathrm{Op}_{h}\left(a_{h}\right) e^{-i t H} \varphi\right\|_{L^{2}\left([-T, T] ; L^{2}\right)} \\
& +C_{T}\left(h 2^{j}\right)^{1 / 2}\left\|\left[\chi_{j} \mathrm{Op}_{h}\left(a_{h}\right), H\right] e^{-i t H} \varphi\right\|_{L^{2}\left([-T, T] ; L^{2}\right)} .
\end{aligned}
$$

Since $2^{j-1} \leq|x| \leq 2^{j+1}$ and $|x| \leq 1 / h$ on $\operatorname{supp} \chi_{j} a_{h}$ we have, for any $\varepsilon \geq 0$,

$$
\begin{aligned}
& \left(h 2^{j}\right)^{-1 / 2}\left\|\chi_{j} \mathrm{Op}_{h}\left(a_{h}\right) e^{-i t H} \varphi\right\|_{L^{2}\left([-T, T] ; L^{2}\right)} \\
& \leq C\left\|\chi_{j}\langle x\rangle^{-1 / 2-\varepsilon} h^{-1 / 2-\varepsilon} \operatorname{Op}_{h}\left(a_{h}\right) e^{-i t H} \varphi\right\|_{L^{2}\left([-T, T] ; L^{2}\right)}
\end{aligned}
$$

Since $\left\{\chi_{j} a_{h}, p\right\} \in S\left(\langle x\rangle^{-1}\langle\xi\rangle, g\right)$, we similarly obtain

$$
\begin{aligned}
& \left(h 2^{j}\right)^{1 / 2}\left\|\chi_{j}\left[\mathrm{Op}_{h}\left(a_{h}\right), H\right] e^{-i t H} \varphi\right\|_{L^{2}\left([-T, T] ; L^{2}\right)} \\
& \leq\left\|\widetilde{\chi}_{j}\langle x\rangle^{-1 / 2-\varepsilon} h^{-1 / 2-\varepsilon} \operatorname{Op}_{h}\left(b_{h}\right) e^{-i t H} \varphi\right\|_{L^{2}\left([-T, T] ; L^{2}\right)}+C_{T} h\|\varphi\|_{L^{2}},
\end{aligned}
$$

where $\tilde{\chi}_{j}(x)=\widetilde{\chi}\left(2^{-j} x\right)$ for some $\tilde{\chi} \in C_{0}^{\infty}\left(\mathbb{R}^{d}\right)$ satisfying $\tilde{\chi} \equiv 1$ on $[1 / 2,2]$ and $\operatorname{supp} \tilde{\chi} \subset[1 / 4,4]$, and $b_{h} \in S(1, g)$ is supported in a neighborhood of $\operatorname{supp} a_{h}$ so that $b_{h} \equiv 1$ on $\operatorname{supp} a_{h}$. Summing over $1 \leq j \leq j_{h}$ and using the local smoothing effect (6.2), since $p, q \geq 2$, we obtain

$$
\begin{aligned}
& \left\|\mathrm{Op}_{h}\left(a_{h}\right) e^{-i t H} \varphi\right\|_{L^{p}\left([-T, T] ; L^{q}\right)}^{2} \\
& \leq \sum_{1 \leq j \leq j_{h}}\left\|\chi_{j} \mathrm{Op}_{h}\left(a_{h}\right) e^{-i t H} \varphi\right\|_{L^{p}\left([-T, T] ; L^{q}\right)}^{2} \\
& \leq C_{T} \sum_{1 \leq j \leq j_{h}}\left(\left\|\chi_{j} \mathrm{Op}_{h}\left(a_{h}\right) \varphi\right\|_{L^{2}}^{2}+h\|\varphi\|_{L^{2}}^{2}\right) \\
& +C \sum_{1 \leq j \leq j_{h}}\left\|\tilde{\chi}_{j}\langle x\rangle^{-1 / 2-\varepsilon} h^{-1 / 2-\varepsilon} \mathrm{Op}_{h}\left(a_{h}+b_{h}\right) e^{-i t H} \varphi\right\|_{L^{2}\left([-T, T] ; L^{2}\right)}^{2} \\
& \leq C_{T}\|\varphi\|_{L^{2}}^{2}+C\left\|\langle x\rangle^{-1 / 2-\varepsilon} h^{-1 / 2-\varepsilon} \mathrm{Op}_{h}\left(a_{h}+b_{h}\right) e^{-i t H} \varphi\right\|_{L^{2}\left([-T, T] ; L^{2}\right)}^{2} \\
& \leq C_{T, \varepsilon} h^{-2 \varepsilon}\|\varphi\|_{L^{2}}^{2},
\end{aligned}
$$

which implies

$$
\begin{aligned}
\sum_{h}\left\|\mathrm{Op}_{h}\left(a_{h}\right) e^{-i t H} f\left(h^{2} H\right) \varphi\right\|_{L^{p}\left([-T, T] ; L^{q}\right)}^{2} & \leq C_{T, \varepsilon} \sum_{h} h^{-2 \varepsilon}\left\|f\left(h^{2} H\right) \varphi\right\|_{L^{2}}^{2} \\
& \leq C_{T, \varepsilon}\left\|\langle H\rangle^{\varepsilon / 2} \varphi\right\|_{L^{2}}^{2} .
\end{aligned}
$$

We complete the proof. 


\section{References}

[1] Bouclet, J.- M.: Littlewood-Paley decompositions on manifolds with ends. Bull. Soc. Math. France. 138 (2010), 1-37.

[2] Bouclet, J.- M.: Semi-classical calculus on non compact manifolds with ends and weighted $L^{p}$ estimates. Ann. Inst. Fourier. 61(2011), 1181-1223.

[3] Bouclet, J.-M.: Strichartz estimates on asymptotically hyperbolic manifolds. Anal. PDE. 4 (2011), 1-84.

[4] Bouclet, J.-M., Tzvetkov, N.: Strichartz estimates for long range perturbations. Amer. J. Math. 129 (2007), 1565-1609.

[5] Bouclet, J.-M., Tzvetkov, N.: On global Strichartz estimates for non trapping metrics. J. Funct. Analysis. 254 (2008), 1661-1682.

[6] Burq, N., Gérard, P., Tzvetkov, N.: Strichartz inequalities and the nonlinear Schrödinger equation on compact manifolds. Amer. J. Math. 126 (2004), 569-605.

[7] Burq, N., Guillarmou, C., Hassell, A.: Strichartz estimates without loss on manifolds with hyperbolic trapped geodesics. Geom. Funct. Anal. 20 (2010), 627-656.

[8] Cazenave, T.: Semilinear Schrödinger equations. Courant. Lect. Nates Math. vol. 10, AMS, Providence, RI, 2003.

[9] D'Ancona, P., Fanelli, L.: Smoothing estimates for the Schrödinger equation with unbounded potentials. J. Differential Equations 246 (2009), 4552-4567.

[10] D'Ancona, P., Fanelli, L., Vega, L., Visciglia, N.: Endpoint Strichartz estimates for the magnetic Schrödinger equation. J. Funct. Analysis 258 (2010), 3227-3240.

[11] Doi, S.: Smoothness of solutions for Schrödinger equations with unbounded potentials. Publ. Res. Inst. Math. Sci. 41 (2005), 175-221.

[12] Erdoğan, M., Goldberg, M., Schlag, W.: Strichartz and smoothing estimates for Schrödinger operators with almost critical magnetic potentials in three and higher dimensions. Forum Math. 21 (2009), 687-722.

[13] Fujiwara, D.: Remarks on convergence of the Feynman path integrals. Duke Math. J. 47 (1980), 559-600.

[14] Ginibre, J., Velo, G.: The global Cauchy problem for the non linear Schrödinger equation. Ann. lHP-Analyse non linéaire. 2 (1985), 309-327.

[15] Hassell, A., Tao, T., Wunsch, J.: Sharp Strichartz estimates on nontrapping asymptotically conic manifolds. Amer. J. Math. 128 (2006), 963-1024.

[16] Helffer, B., Sjöstrand, J.: Equation de Schrödinger avec champ magnétique et équation de Harper. In Schrödinger Operators, H. Holden and A. Jensen, eds., pp. 118-197. Lecture Notes in Physics 345, Springer-Verlag, 1989 
[17] Isozaki, H., Kitada, H.: Modified wave operators with time independent modifiers. J. Fac. Sci. Univ. Tokyo. 32 (1985), 77-104.

[18] Journé, J.- L., Soffer, A., Sogge, C. D.: Decay estimates for Schrödinger operators. Comm. Pure Appl. Math. 44 (1991), 573-604.

[19] Keel, M., Tao, T.: Endpoint Strichartz Estimates. Amer. J. Math. 120 (1998), 955-980.

[20] Kitada, H., Kumano-go, H.: A family of Fourier integral operators and the fundamental solution for a Schrödinger equation. Osaka J. Math. 18 (1981), 291-360.

[21] Martinez, A.: An Introduction to Semiclassical and Microlocal Analysis. New York: Universitext, Springer-Verlag, 2002

[22] Marzuola, J., Metcalfe, J., Tataru, D.: Strichartz estimates and local smoothing estimates for asymptotically flat Schrödinger equations. J. Funct. Analysis. 255 (2008), 1497-1553.

[23] Mizutani, H.: Strichartz estimates for Schrödinger equations on scattering manifolds. Comm. Partial Differential Equations. 37 (2012), 169-224.

[24] Mizutani, H.: Strichartz estimates for Schrödinger equations with variable coefficients and potentials at most linear at spatial infinity, to appear in J. Math. Soc. Japan. (http://arxiv.org/abs/1108.2103)

[25] Robbiano, L., Zuily, C.: Strichartz estimates for Schrödinger equations with variable coefficients. Mém. SMF. Math. Fr. (N.S.), No. 101-102, 1-208 (2005)

[26] Robert, D.: Autour de l'approximation semi-classique. Progr. Math. 68 Birkhäuser, Basel, 1987

[27] Robert, D.: Relative time delay for perturbations of elliptic operators and semiclassical asymptotics. J. Funct. Analysis 126 (1994), 36-82.

[28] Robert, D., Tamura, H.: Semi-classical estimates for resolvents and asymptotics for total scattering cross-sections. Ann. Inst. Henri Poincare 46 (1987), 415-442.

[29] Schlag, W.: Dispersive estimates for Schrödinger operators: a survey. Mathematical aspects of nonlinear dispersive equations, Ann. of Math. Stud. Princeton Univ. Press, Princeton, NJ. 163 (2007), 255-285

[30] Staffilani, G., Tataru, D.: Strichartz estimates for a Schrödinger operator with non smooth coefficients. Comm. Partial Differential Equations. 27 (2002), 1337-1372.

[31] Strichartz, R.: Restrictions of Fourier transforms to quadratic surfaces and decay of solutions of wave equations. Duke Math. J. 44 (1977), 705-714.

[32] Tataru, D.: Parametrices and dispersive estimates for Schrödinger operators with variable coefficients. Amer. J. Math. 130 (2008), 571-634.

[33] Yajima, K.: Existence of solutions for Schrödinger evolution equations. Comm Math. Phys. 110 (1987), 415-426. 
[34] Yajima, K.: Schrödinger evolution equation with magnetic fields. J. d'Anal. Math. 56 (1991), 29-76.

[35] Yajima, K.: Boundedness and continuity of the fundamental solution of the time dependent Schrödinger equation with singular potentials. Tohoku Math. J. 50 (1988), 577-595.

[36] Yajima, K.: Dispersive estimates for Schrödinger equations with threshold resonance and eigenvalue. Comm. Math. Phys. 259 (2005), 475-509.

[37] Yajima, K., Zhang, G.: Local smoothing property and Strichartz inequality for Schrödinger equations with potentials superquadratic at infinity. J. Differential Equations. 202 (2004), 81-110. 